\title{
N $93-14772$
}

TRIBOLOGY OF MAGNETIC STORAGE SYSTEMS

\author{
Bharat Bhushan \\ Ohio Eminent Scholar Professor \\ Director, Computer Microtribology and Contamination Laboratory \\ Department of Mechanical Engineering \\ Ohio State University \\ Columbus, Ohio 43210
}

"Limited Distribution Notice: This report has been submitted for publication and will probably be copyrighted if accepted for publication. It is being reproduced here for early disseminiation of its contents. In view of the possible transfer of the copyright to the outside publisher, its distribution. prior to outside publication, should be limited to peer communication and specific requests. After outside publication, requests should be filled only by reprints from the outside publication, or legally obtained coples of the article." 



\subsection{Physics of Magnetic Recording}

Recording technology is founded on magnetism and on electromagnetic induction. It is well known that currents in electric wires produce a magnetic field that can magnetize a hard magnetic material permanently. This permanent magnet can, in turn, generate an electric voltage: if it is dropped through a coll, it will generate a voltage pulse. We have just described the basic principle of magnetic recording (writing) and playback (reading), and it is shown schematically in Fig. 1, which consists of the relative motion between a magnetic medium and a read/write ring head (Lowman, 1962; Hoagland, 1963; Camras, 1988; Jorgensen, 1988; Mee and Daniel, 1990). Read/write heads (inductive type) consist of a ring of high-permeability magnetic material with an electrical winding and a gap in the magnetic material at or near the surface of the storage medium. Writing is accomplished by passing a current through the coil. The flux is confined to the magnetic core, except in the region of the small nonmagnetic gap. The fringe field in the vicinity of the gap, when sufficiently strong, magnetizes the medium, moving past the write head. The magnetic medium consists of high-coercivity magnetic materlal that retains its magnetization after it has passed through the field from the write head gap. The medium passes over the read head, which, like the write head, is a ring core with an air gap. Each particle in the medium is a miniature magnet, and its flux lines will add up with those of the other particles to provide an external medium flux, proportional in magnitude to the medium magnetization. The flux lines from the medium permeate the core and induce a voltage in the head winding. This voltage, after suitable amplification, reproduces the original signal. A single head can be used for both read and write functions.

More recently, some read heads are of the magnetoresistive (MR) type in which a strip of a ferromagnetic alloy (for example. $\mathrm{Ni}_{80} \mathrm{Fe}_{20}$ ) is mounted vertically. The variation of the magnetic-field component in the magnetic medium (perpendicular to the plane of medium) causes the variation in the electrical resistance of the MR stripe which can be readily measured (Van Gestel et al., 1977). MR-type read heads are attractive because they can be miniaturized without reducing the sensitivity to an unacceptably low value. One disadvantage of the MRtype head over the Inductive-type head is that both read and write functions cannot be combined in one head (Bhushan, 1990).

So far, we have described longitudinal (horizontal) recording. In 1977, perpendicular (vertical) recording was proposed for ultrahigh density magnetic recording by Iwasaki and Nakamura (1977). In vertical recording, magnetization is orlented perpendicular to the plane of medium rather than in its plane. Single-pole heads are generally used for recording on vertical media. Ring-shaped heads, previously discussed, have also been used with some design changes. Vertical recording has the advantage of reduced self-demagnetization (Bhushan, 1990).

For high areal recording density, the linear flux density (number of flux reversals per unit distance) and the track density (number of tracks per unit distance) should be as high as possible. Reproduced (read) signal amplitude decreases with a decrease in the recording wavelength and/or the track width. The signal loss occurs from the magnetic coating thickness, head-to-medium spacing (clearance or flying height) and read gap length. The spacing loss of interest here was first described by Wallace (1951),

$$
S(\lambda)=\exp (-2 \pi d / \lambda) \text { or }-54.6 d / \lambda \mathrm{dB}
$$

where $\lambda$ is the recording wavelength and $d$ is the head-medium separation. We note from Eq. 1 that the spacing loss can be reduced exponentially by reducing the separation between the head and the medium. The noise in the reproduced signal needs to be minimized. Thus signal-tonoise ratio (SNR) must be as high as possible. The wide-band SNR is also dependent upon headto-medium spacing. 


\subsection{Importance of Tribology}

We have Just stated that the magnetic recording process is accomplished by relative motion between magnetic media against a stationary (audio and data processing) or rotating (audio, video and data processing) read/write magnetic head. The heads in modern magnetic storage systems are designed so that they develop hydrodynamic (self-acting) air bearing under steady operating condition. Formation of air bearing minimizes the head-medium contact. Physical contact between the medium and the head occurs during starts and stops. In modern high-end data-storage tape and disk drives, the head-to-media separation is on the order of 0.1 to $0.3 \mu \mathrm{m}$, and the head and medium surfaces have roughness on the order of 2 to $10 \mathrm{~nm}$ rms (Bhushan, 1990, 1992a). The need for higher and higher recording densities requires that surfaces be as smooth as possible and flying height be as low as possible. Smoother surfaces lead to increase in adhesion, friction, and interface temperatures and closer flying heights lead to occasional rubbing of high asperities and increased wear. Friction and wear issues are resolved by appropriate selection of interface materials and lubricants, by controlling the dynamics of the head and medium, and the environment. A fundamental understanding of the tribiology of magnetic head medium interface becomes cruclal for the continued growth of the magnetic storage industry which is currently a $\$ 50$ billion a year industry.

This chapter starts out to define the construction and the materials used in different magnetic storage devices. It then presents the theories of friction and adhesion, interface temperatures, wear, and solid-liquid lubrication relevant to magnetic storage systems. Experimental data are presented wherever possible to support the relevant theories advanced in this chapter.

\subsection{MAGNETIC STORAGE SYSTEMS}

\subsection{Examples of Modern Systems}

Magnetic Storage devices used for information (audio, video and data processing) storage and retrieval are: tape, nexible disk and rigid disk drives (Bhushan, 1990, 1992a).

\subsection{Tape Drives}

Linear analog technique is most commonly used for most domestic audio recorders which use typically a tape with a $4 \mathrm{~mm}$ width. There is always a physical contact between the head and the tape. Rotary single-track heads developed for video recording are also used for high recording density capabilities. For professional recording, dedicated digital technologies have emerged based, again, on either a multi-track stationary-head (S-DAT) approach or a rotating-head (R-DAT) approach.

A video recorder uses a helical-scanning rotating-head configuration. Because a rotating head can be moved at a greater speed than a heavy roll of tape, much higher data rates can be achleved in a rotating head drive than a linear tape drive. A $12.7-\mathrm{mm}$ or 8-mm wide tapes are most commonly used. For professional video recording, digital video recorders are used for high signal-to-noise ratio.

Digital tape drives are used for data-processing (computer) applications. Figure 2 shows a schematic of the high-density, high-data-rate, IBM 3480/3490 tape drive for mainframe computers. For this drive, a $165-\mathrm{m}$ long, $12.7-\mathrm{mm}$ wide, and roughly $26-\mu \mathrm{m}$ thick particulate tape wound on a reel is housed inside a rectangular cartridge (100 x $125 \times 25 \mathrm{~mm})$. A schematic of the 18-track read-write thin-film head is shown in Fig. 3. The write head is an inductive type and the read head is a MR type. The bleed slots are provided to reduce the flying height for maximum reproduced amplitude. Edge slots are provided for flying uniformity. 
Helical scanning rotary-head configuration in a $8-\mathrm{mm}$ tape format similar to video recorders and in a 4-mm tape format same as R-DAT audio recorders are used in tape drives; 8$\mathrm{mm}$ format is used for very high volumetric density for mid-range computers (work stations). Drives using 130-mm full height form factors are commonly used. Belt-driven (longitudinal) data cartridges are commonly used for relatively high capacity in mid-range computers. A data cartridge tape is a self-contained reel-to-reel tape deck without a motor or read-write head. The tape width is $6.35-\mathrm{mm}$. The overall dimensions of the cartridge are $152 \mathrm{~mm} \times 102$ $\mathrm{mm} \times 17 \mathrm{~mm}$ and $80 \mathrm{~mm} \times 61 \mathrm{~mm} \times 15 \mathrm{~mm}$ for $130-\mathrm{mm}$ half-height and $95-\mathrm{mm}$ half-height form factors, respectively. The 4 -mm reel-to-reel data cassettes similar to audio cassette recorders are used for relatively small computers. These are also packaged in 95- $\mathrm{mm}$ and 130$\mathrm{mm}$ half-height form factors.

\subsubsection{Flexible Disk Drives}

Flexible disk, also called a floppy disk or diskette is a magnetic recording medium, which is physically a thin $(-82-\mu \mathrm{m}$ thickness) and pliable disk and functionally a removable, random-access cartridge. When mounted in the drive, the disk is clamped at its center and rotated at a relatively low speed while the read-write head accesses the disk through a slot in the jacket. In most designs the accessing arms traverse above and below the disk and with readwrite head elements mounted on spring suspensions. Head positioning is usually accomplished by a stepping motor, Fig. 4. The flexible disk heads are either spherically contoured or are flat in shape. Most commonly used disk drives today are in 90-mm (3.5 in.) and $130-\mathrm{mm}(5.25 \mathrm{in}$.) form factors which use disk diameters, of $85.8 \mathrm{~mm}$ and $130.2 \mathrm{~mm}$, respectlvely. The $50-\mathrm{mm}$ ( $2 \mathrm{in}$.) form factor drives use $47-\mathrm{mm}$ or $50.8-\mathrm{mm}$ diameter disks and these drives are used in "notebook" computers. The $90-\mathrm{mm}$ form factor disks use a metal hub and are encased in a hard plastic jacket which does not bend like the $130-\mathrm{mm}$ form factor soft jackets and incorporates a shutter to protect the disk surface. A so-called "Bernoullie" disk drive has been developed by Iomega Corp., Roy. Utah to obtain the stable interface at higher operating speeds. In this design, the flexible disk is rotated at high speed in close proximity to a fixed flat plate called the Bernoullie plate. A foundation stiffness results from hydrodynamic loading (air bearing) created by spinning the disk in close proximity to the base plate. A foundation stiffness results from hydrodynamic loading (air bearing) created by spinning the disk in close proximity to the base plate. The high disk stiffness pushes up the first critical speed. Therefore, these disks can he operated at higher speeds (> $1500 \mathrm{rpm})$ without disk flutter.

\subsubsection{Rigid Disk Drives}

The rigid disk drive technology commonly referred to as "Winchester" technology uttlizes the rigid disks as a nonremovable stack in a drive. The disks rotate at a constant angular speed. with concentric data tracks recorded on their surfaces. The heads are moved by an actuator that positions each head over a desired data track. Typically one or two heads (for fast accessing) are moving over each disk surface. While all heads are actuated together, only one head is selected at a time to read or write. The schematic of the head-disk interface of a high density, high-data rate, IBM 3390-type disk drive with a linear actuator driven by a voice coil motor is shown in Fig. 5.

Fairly large motors are used to drive the precision spindle holding the disks. A high starting torque is needed in order to overcome the static friction from often many heads resting on the disks. Hence brushless DC motors are used, mounted inside the spindle or underneath the base plate casting. Preloaded and sealed ball bearings are used to support the spindle shaft in both ends.

Conventionally, a slider is mounted on a flexure in the orientation optimal for linear actuators. The longitudinal axis of flexure points is in the direction of carriage actuation, with 
the slider mounted at a right angle. However, current trends are toward smaller, more compact disk storage devices, especlally in the low-end applications. The compact, low mass, low-cost rotary actuators are used to save space in the drive. In rotary actuators, the slider is mounted along the rotary arm. The actuator is operated by a stepping motor or voice-coil motor (VCM), Figs. 4 and 5. The VCM is very much like a loudspeaker coil/magnet mechanism. The VCM has the advantage of providing the desired linear or the rotary motion directly whereas circular motion provided by the stepping motor needs to be converted to the desired linear or rotary motion generally by a lead screw or capstan-band (Fig. 4) method. Furthermore, the VCM provided faster and smaller stepping than that by stepping motor. The small drives use both linear and rotary actuators driven by either stepping or VCM motor. The large drives use a linear actuator driven by a VCM. The actuator connected to the VCM, rides on a set of ball hearings on the tracks as shown in fig. 5.

The head slider used in high-end rigid disk drive (IBM 3380K/ 3390) is a two-shaped rail, taper-flat design supported by a leaf spring (flexure) suspension, made of nonmagnetic steel to allow motion along the vertical, pitch and roll axes, Fig. 6. The front taper serves to pressurize the alr lubricant, while some of the air is lost through leakage to the side boundaries of the rall resulting in a pitch angle. In the shaped-rail design, each side rall has a widened leading-edge rail width which is flared down to a smaller rail width towards the trailing end, Fig. 6(a). Shaped-rall design is used to attain increased pitch angles, independent of airfilm thickness. The inductive-type thin-film read-write elements, located at the trailing edge of each rall, are an integral part of the sllder where the lowest flying height occurs. Suspension supplies a vertical load of either $100 \mathrm{mN}(10 \mathrm{~g})$ or $150 \mathrm{mN}(15 \mathrm{~g})$ which is balanced by the hydrodynamic load when the disk is spinning. The stiffness of the suspension $\left(\sim 25 \mathrm{mN} \mathrm{mm}^{-1}\right)$ is several orders of magnitude lower than that of the air bearing $\left(-0.5 \mathrm{kN} \mathrm{mm}^{-1}\right)$ developed during use so that most dynamic varlations are taken up by the suspension without degrading the air bearing.

Small disk drives use inductive-coil type heads. Two types of head sliders are most commonly used: minimonolithic (mini-Winchester) and minicomposite. A minimonolithic head slider consists of a slider body and a core piece carrying the coil, both consisting of monolithic magnetic material. It is a tri-rail design. The taper-flat bearing area is provided by the outer two ralls of the tri-rall design. The center rall defines the width of the magnetic element in the tralling edge where a ferrite core is formed. A minimonolithic head slider consists of a Mn-Zn ferrite core with read-write gap. glass bonded into air-bearing surface of a nonmagnetic, wear-resistant slider (typically calcium titanate) of approximately the same size as minimonolithic slider. The 3380-type suspensions are normally used for heads in small drtves and apply a $95 \mathrm{mN}(9.5-\mathrm{g})$ load onto the slider.

The 3380-K/3390 type sliders are about $4.045-\mathrm{mm}$ long by $3.200-\mathrm{mm}$ wide by $0.850 \mathrm{~mm}$ high with a mass of $0.45 \mathrm{mN}(45 \mathrm{mg})$, as opposed to about $4.1 \mathrm{~mm}$ long by 3.1 -nun wide by 1.4 $\mathrm{mm}$ high and $0.7 \mathrm{mN}(70 \mathrm{mg})$ for the minl Winchester. The surface roughness of the air-bearing ralls is typically $1.5-2.5 \mathrm{~nm}$ mins.

Schematic representations of head-medium interfaces for tape, flexible, and rigid-disk drives are shown in Fig. 7. We note that the environment, usage time, and contamination (external and wear debris) play a significant role in the reliability of the interface.

\subsection{Magnetic Head and Medium Materlals}

\subsubsection{Magnetic Heads}

Magnetic heads used to date are either conventional inductive or thin-film inductive and magnetoresistive (MR) heads. Trends to film-head design have been driven by the desire to capitalize on semi-conductor-like processing technology to reduce fabrication costs. In addition, thin-film technology allows the production of high-track density heads with 
accurate positioning control of the tracks and high reading sensitivity. Conventional heads are combination of a body forming the air bearing surface and a magnetic ring core carrying the wound coll with a read-write gap. In the film heads, the core and colls or MR stripes are deposited by thin-film technology. The alr-bearing surfaces of tape heads are cylindrical in shape. The tape is slightly underwrapped over the head surface to generate hydrodynamic lift during read-write operation. For inductive-coil tape heads, the core materials that have been typically used are permalloy and Sendust. However, since these alloys are good conductors, it is sometimes necessary to laminate the core structure to minimize losses due to eddy currents. The air-bearing surfaces of most inductive-coil type heads consist of plasma sprayed coatings of hard materials such as $\mathrm{Al}_{2} \mathrm{O}_{3}-\mathrm{TiO}_{2}$ and $\mathrm{ZrO}_{2}$. Read and write heads in modern tape drives (such as IBM 3480/3490) are minlaturized using thin-film technology, Fig. 3. Film heads are generally deposited on $\mathrm{Ni}-\mathrm{Zn}$ ferrite ( 11 wt \% $\mathrm{NiO}, 22$ wt \% $\mathrm{ZnO}, 67$ wt \% $\left.\mathrm{Fe}_{2} \mathrm{O}_{3}\right)$ substrates. Flexible-disk heads are inductive-coll type composite heads which are spherically contoured or are flat in shape. $\mathrm{Mn}-\mathrm{Zn}$ ferrite ( $30 \mathrm{wt} \% \mathrm{MnO}, 17 \mathrm{wt} \% \mathrm{ZnO}, 53 \mathrm{wt} \% \mathrm{Fe}_{2} \mathrm{O}_{3}$ ) is generally used for head cores and barium titanate is generally selected for the magnetically inert structures which support the cores.

The material used in the construction of thin-film (Winchester-type) head used in large disk drives is generally (nonmagnetic) $\mathrm{Al}_{2} \mathrm{O}_{3}-\mathrm{TiC}$ (70-30 wt \%). Some manufacturers use yttria-stabilized zirconia/alumina - titanium carbide composite. Small rigid-disk drives for low-end applications use heads with magnetic ring core and a wound coll. Two types of head sliders are most commonly used: minimonolithic (or mini-Winchester) and minicomposite. A minimonolithic head slider consists of a slider body and a core piece carrying the coil, both consisting of monolithic magnetic material, typically $\mathrm{Mn}-\mathrm{Zn}$ ferrite. A minicomposite head slider consists of a $\mathrm{Mn}-\mathrm{Zn}$ ferrite core with read-write gap. glass bonded into the airbearing surface of a nonmagnetic, wear-resistant slider (typically calcium titanate). Typical physical properties of hard head materials are presented in Table 1.

\subsubsection{Magnetic Media}

Magnetic media fall into two categories: particulate media, where magnetic particles are dispersed in a polymeric matrix and coated onto the polymeric substrate for flexible media (tape and flexible disks) or onto the rigid substrate (typically aluminum, more recently introduced glass for rigid-disks); thin-film media, where continuous films of magnetic materials are deposited onto the substrate by vacuum techniques. Requirements of higher recording densitles with low error rates have resulted in an increased use of thin films which are smoother and considerably thinner than the particulate media. The thin-film media is extensively used for rigid-disks and has begun to be used for high.density audio/video and data processing tape applications.

Sectional views of a particulate and a thin-film (evaporated) metal tape are shown in Fig. 8. Flexdble disks are similar to tapes in construction except these are coated with magnetic coating on both sides and the substrate is generally about $76.2 \mu \mathrm{m}$ in thickness. The base film for flexible media is almost exclusively poly(ethylene terephthalate) (PET) film. Other substrate materials such as polyimides and polyamide-polyimide copolymers have been explored for better dimensional stability of flexible disks. Typically a 6.35 to $36.07-\mu \mathrm{m}$ thick $(25,35,57,88,92$, and 142 gage) PET substrate with an rms roughness of about 1.5 to $2.5 \mathrm{~nm}$ for particulate media and even smoother (1.5 to $2 \mathrm{~nm} \mathrm{~ms})$ for thin-films media is used for tapes $(14.48-\mu \mathrm{m}$ or thicker for data processing applications) and 76.2- $\mu \mathrm{m}$ thick (300 gage) for flexible disks. The base film is coated with a magnetic coating, typically 2 to $4 \mu \mathrm{m}$ thick (coated on both sides with a magnetic coating, in case of flexible disks), composed by acicular magnetic particles Isuch as $\gamma-\mathrm{Fe}_{2} \mathrm{O}_{3}, \mathrm{Co}$-modified $\gamma-\mathrm{Fe}_{2} \mathrm{O}_{3}, \mathrm{CrO}_{2}$ (only for tapes), and metal particles for horizontal recording or hexagonal platelets of barlum ferrite for both horizontal and vertical recording], polymeric binders [such as polyester-polyurethane, polyether-polyurethane, nitrocellulose, poly(vinyl chloride), poly(vinyl alcohol-vinyl acetate), poly(vinylidene chloride), VAGH, phenoxy, and epoxy, lubricants (mostly fatty acid esters, e.g., tridecyl stearate, butyl stearate, butyl palmitate, butyl myristate, stearic acid, myrstic acid), a cross 
linker or curing agent (such as functional isocyanates), a dispersant or wetting agent (such as lecithin), and solvents (such as tetrahydrofuran and methyl isobutylketone). In some media, carbon black is added for antistatic protection if the magnetic particles are highly insulating and abrasive particles (such as $\mathrm{Al}_{2} \mathrm{O}_{3}$ ) are added as a head cleaning agent and to improve media's wear resistance. Magnetic particles are typically $70-80 \%$ by weight (or $43-50 \%$ by volume), lubricants are typically $1-3 \%$ by weight of the total solid coating. The coating is calendered to a surface roughness of 8 to $15 \mathrm{~nm} \mathrm{mms}$.

Most magnetic tapes have a 1 to $3-\mu \mathrm{m}$ thick backcoating for antistatic protection and for improved tracking. The backcoat is generally a polyester-polyurethane coating containing conductive carbon black and $\mathrm{THO}_{2}$. The $\mathrm{TiO}_{2}$ and carbon contents are typically $50 \%$ and $10 \%$ by weight, respectively.

Flexible disks are packaged inside a soft polyvinyl chloride (PVC) jacket or a acrylonitrile-butadiene-styrene (ABS) hard jacket (for smaller $90-\mathrm{mm}$ form factor). Inside the jacket, a soft liner, a protective fabric is used to minimize wear or abrasion of the media. The wiplng action of the liner on the medium coating removes and entraps particulate contaminants which may originate from the diskette manufacturing process, the jacket, headdisk contact (wear debris), and the external environment. The liner is made of nonwoven fibers of polyester (PET), rayon, polypropylene or nylon. The liner fibers are thermally or fusion bonded to the plastic jacket at spots. The soft jacket near the data window is pressed with a sponge pad to create a slight friction and hence stabilize the disk motion under the heads. The hard cartridge is provided with an internal plastic leaf spring for the same purpose.

Thin-film (also called metal-film) flexible media consist of polymer substrate (PET or polyimide) with an evaporated film of Co-Ni (with about $18 \% \mathrm{NI}$ ) and less commonly evaporated/sputtered $\mathrm{Co}-\mathrm{Cr}$ (with about $17 \% \mathrm{Cr}$ ) for vertical recording, which is typically 100 $300 \mathrm{~nm}$ thick. Electroplated Co and electroless plated Co-P, Co-Ni-P and Co-Ni-Re-P have also been explored but are not commerclally used. Since the thickness of the magnetic layer is only $100-300 \mathrm{~nm}$, the surface of the thin-film medium is greatly influenced by the surface of the substrate fllm. Therefore, an ultra-smooth PET substrate film (rms roughness $-1.5-2 \mathrm{~nm}$ ) is used to obtain a smooth tape surface (rms roughness $\sim 5-6 \mathrm{~nm}$ ) for high-density recording. Several undercoatings, overcoatings and oxidation treatments (by adding oxygen into the vacuum chamber during evaporation) are used to increase corrosion resistance and durability. An undercoating layer has been employed consisting of very fine particles, each protruding few nanometers. Varlous organic (such as acrylic polymer in 50-80 nm thickness) and inorganic overcoats (such as diamondlike carbon in about 10-20 nm thickness, $\mathrm{SiO}_{2}$ and $\mathrm{ZrO}_{2}$ ) have been used to protect against corrosion and wear. Alumina $(0.2 \mu \mathrm{m})$ particles are also added in some polymeric overcoats. Yet in another approach oxygen is leaked into the vacuum chamber at a controlled rate during the Co-Ni evaporation. This has the effect of forming an oxide top layer of 10 to $30 \mathrm{~nm}$, and probably introduces oxidized grain boundartes. This oxidation improves durability and corrosion resistance and decreases magnetic noise. Surface oxidation of evaporated $\mathrm{Co}-\mathrm{Cr}$ coating by annealing in air has also been reported to improve the durability of the coating. In addition to a solid overcoat, generally a thin layer (2-10 nm in thickness or $4-20 \mathrm{mg} / \mathrm{m}^{2}$ in weight) liquid lubricant is applied on the medium surface to further reduce friction, wear and corrosion. Fatty acid esters or fluorine-based compounds (such as perfluoropolyethers) are most commonly used (Bhushan, 1992a).

Figure 9 shows sectional views of two types of rigid disks - a particulate disk and a thinfilm disk. The substrate for rigid disks is generally a non heat-treatable aluminummagnesium alloy AlSi $5086(95.4 \% \mathrm{Al}, 4 \% \mathrm{Mg}, 0.4 \% \mathrm{Mn}$, and $0.15 \% \mathrm{Cr})$ with an ms surface roughness of about $15-25 \mathrm{~nm}$ rms and a Vickers hardness of about $90 \mathrm{~kg} / \mathrm{mm}^{2}$. For particulate disk, the Al-Mg substrate with a thickness of 1.3 to $1.9 \mathrm{~mm}$, is sometimes passivated with a very thin $(<100 \mathrm{~nm})$ chromium conversion coating based on chromium phosphate. The finished substrate is spin coated with the magnetic coating about 0.75 to $2 \mu \mathrm{m}$ thick and burnished to a surface roughness of about 7.5 to $15 \mathrm{~nm}$ ms. The binder system generally used is a hard copolymer of epoxy, phenolic and polyurethane constituents. About 30 to $35 \%$ by 
volume of acicular magnetic particles of $\gamma-\mathrm{Fe}_{2} \mathrm{O}_{3}$ are interdispersed in the binder. A small percentage ( 2 to $8 \%$ by volume) of $\mathrm{Ai}_{2} \mathrm{O}_{3}$ particles $0.2-0.5 \mu \mathrm{m}$ in size are added to improve the wear resistance of the magnetic coating. A thin film of perfluoropolyether lubricant (3-6 nm thick) is applied topically. The magnetic coating is made porous for lubricant retention (Bhushan, 1990).

For high-density recording, trends are to use thin-film disks. For thin-fllm disks with a metallic magnetic layer, the Al-Mg substrate with a thickness of 0.78 to $1.3 \mathrm{~mm}$, is electroless plated with a 10- to 20- $\mathrm{mm}$ thick nickel-phosphorous (90-10 wt \%) layer to improve its surface hardness to $600-800 \mathrm{~kg} / \mathrm{mm}^{2}$ (Knoop) and smoothness. The coated surface is polished with an abrastve slurry to a surface roughness of about 2 to $4 \mathrm{~nm}$ rms. For a thin-film disk with an oxide magnetic layer, a $2-$ to $20-\mu \mathrm{m}$ thick alumite layer is formed on the Al-Mg substrate through anodic oxidation in a $\mathrm{CrO}_{3}$ bath. In this application, Ni-P cannot be used because it becomes magnetic when exposed to high temperature during preparation of $\gamma-\mathrm{Fe}_{2} \mathrm{O}_{3}$ film. Start-stop zone of substrates for thin-film are generally textured mechanically in the circumferential or random orlentation to a rms roughness typically ranging from $6-8 \mathrm{~nm} \mathrm{mms}$ in order to minimize static friction at the head-disk interface. For convenience, the entire disk is generally textured. Circumferential direction of texturing in the data zone (if textured) is preferred in order to keep the magnetic orientation ratio as high as possible. The finished substrate is coated with a magnetic film 25-150 nm thick. Some metal films require a $\mathrm{Cr}$ undercoat ( 10 to $50 \mathrm{~nm}$ thick) as a nucleation layer to improve magnetic properties, such as coercivity. Typically, magnetic films that have been explored are metal fiims of cobalt-based alloys, with sputtered iron oxide being the principal exception. Magnetic films that are used to achieve the high recording density have weak durability and poor corrosion resistance. Protective overcoats with a liquid lubricant overlay are generally used to provide low friction, low wear, and corrosion resistance. Protective coatings, ranging in thickness from $20-40 \mathrm{~nm}$, generally used are sputtered diamondlike carbon, spin coated or sputtered $\mathrm{SiO}_{2}$, sputtered yttria-stabllized zirconla and plasma-polymerized protective (PPP) films. In most cases, a thin layer of perfluoropolyether lubricant (1-4 nm thick) is used (Bhushan, 1990).

Typical materials used for various magnetic media and operating conditions for dataprocessing applications are shown in Table 2. Typical physical properties of components of magnetic media are presented in Table 3.

\subsection{FRICTION AND ADHESION}

When two surfaces come in contact under load, the contact takes place at the tips of the asperities and the load is supported by the deformation of the contacting asperities, Fig. 10. The proximity of the asperitles results in adhesive contacts caused by interatomic attractions. In a broad sense, adhesion is considered to be either physical or chemical in nature. Experimental data suggest that adhesion is primarly due to weak van der Walls forces. When the two surfaces (in contact) move relative to each other, frictional force, commonly referred to as "intrinsic" or "conventional" frictional force, is contributed by adhesion and deformation (or hysteresis). For most practical cases, adhesional friction is the primary contributor (Bhushan, 1990).

In addition, "stiction" can occur due to meniscus/viscous effects, microcapillary evacuation, and changes in surface chemistry (Bhushan et al., 1984a, 1984b; Bradshaw and Bhushan, 1984: Bradshaw et al., 1986). Here we will concentrate on the meniscus/viscous effects only. Generally, any liquid that wets or has a small contact angle on surfaces will condense from vapor in the form of an annular-shaped capillary condensate in the contact zone. The pressure of the liquid films of the capillary condensates or preexisting film of lubricant can significantly increase the adhesion between solid bodies. Liquid-mediated adhesive forces can be divided into two components: meniscus force $\left(F_{M}\right)$ due to surface tension and a rate-dependent viscous force (FV). The total tangential force $F$ required to 
separate the surfaces by sliding is equal to an intrinsic force $\left(F_{A}\right)$ and stiction force ( $\left.F_{S}\right)$ (combination of friction force due to meniscus effect and the peak viscous force)

$$
F=F_{A}+F_{S}=f_{r}\left(W+F_{M}\right)+F_{V}
$$

where $f_{r}$ is true static coefficient of friction and $W$ is the normal load.

Our analysis shows that normal force required to move two flat, well polished surfaces (such as magnetic head and medium surfaces) apart in the presence of liquid medium and/or sticky substance, can be large (up to several $N$ in extreme cases). Therefore, we define the difference between stiction and conventional static and kinetic friction being that stiction requires a measurable normal force (normally several $\mathrm{mN}$ or higher) to pull the two surfaces apart from the static conditions.

\subsection{Conventional Friction}

From Tabor's classical theory of adhesion, frictional force due to adhesion $\left(\mathrm{F}_{\mathrm{A}}\right)$ is defined as follows (Bowden and Tabor, 1950):

$$
\begin{gathered}
\text { for dry contact, } F_{A}=A_{r} \tau_{a} \\
\text { for lubricated contact, } F_{A}=A_{r}\left[\alpha \tau_{a}+(1-\alpha) \tau_{\ell}\right]
\end{gathered}
$$

and

$$
\tau_{\ell}=\eta_{\ell} \mathrm{V} / \mathrm{h}
$$

where $A_{r}$ is the real area of contact, $\alpha$ is the fraction of unlubricated area, $\tau_{\mathbf{a}}$ and $\tau_{\ell}$ are the shear strengths of the dry contact and of the lubricant film, respectively, $\eta_{\ell}$ is the absolute viscosity of the lubricant, $\mathrm{V}$ is the relative sliding velocity, and $\mathrm{h}$ is the lubricant film thickness.

\subsubsection{Greenwood and Williamson's Contact Model}

The contacts can be either elastic or plastic which primarlly depend on the surface topography and the mechanical properties of the mating surfaces. The classical model of elastic-plastic contact between rough surfaces is that of Greenwood and Williamson (1966) (G\&W), which assumed the surface to be composed of hemispherical asperities of uniform size with their heights following a Gaussian distribution about a mean plane. The radius of these asperities is assumed to be equal to the mean radius of curvature that is obtained from roughness measurements. The expression for real area of contact for elastic (e) and plastic (p) contacts are (Bhushan, 1984),

$$
\begin{gathered}
\mathrm{A}_{\mathrm{re}} / \mathrm{A}_{\mathrm{a}} \mathrm{Pa}_{\mathrm{a}} \sim 3.2 / \mathrm{E}_{\mathrm{c}}\left(\sigma_{\mathrm{p}} / \mathrm{R}_{\mathrm{p}}\right)^{1 / 2} \\
\text { or } \psi_{\mathrm{p}}<1.8 \text { or } \psi<0.6 \text {, elastic contact } \\
\mathrm{A}_{\mathrm{re}} / \mathrm{pa}_{\mathrm{a}} \mathrm{A}_{\mathrm{a}}=1 / \mathrm{H} \\
\text { or } \psi_{\mathrm{p}}>2.6 \text { or } \psi>1, \text { plastic contact }
\end{gathered}
$$

and 


$$
\begin{gathered}
\psi_{\mathrm{p}}=\left(\mathrm{E}_{\mathrm{c}} / \mathrm{Y}\right)\left(\sigma_{\mathrm{p}} R_{\mathrm{p}}\right)^{1 / 2}, \text { for polymers } \\
\psi=\left(\mathrm{E}_{\mathrm{c}} / \mathrm{H}\right)\left(\sigma_{\mathrm{p}} / \mathrm{R}_{\mathrm{p}}\right)^{1 / 2}, \text { for metals } / \text { ceramics }
\end{gathered}
$$

where $A_{a}$ is the apparent area of contact; $P_{a}$ is the apparent pressure $\left(W / A_{a}\right)$; $E_{c}$ is the composite modulus of elasticity, $\mathrm{H}$ and $\mathrm{Y}$ are the hardness and yleld strength of the softer material, $\sigma_{p}$ and $R_{p}$ are the composite standard deviation and radius of curvature of the surface summits, and $\psi$ is the plasticity index. $\sigma_{p}$ and $R_{p}$ depend on the instrument resolution and hence are not unique.

Equation 3 for elastic and plastic contacts in the case of metals/ ceramics is plotted in Fig. 11 for better visualization of dependence of $A_{r}$ on $\psi$. We note that the plastic contact results in a minimum contact area. However, repeated plastic contact would lead to an undesirable permanent deformation and smoothening resulting in elastic contacts (and a higher real area of contact). Wear is more probable when asperities touch plastically than in pure elastic contacts. Therefore, it is desirable to design components in the elastic contact regime with $\psi$ close to the elastic contact limit $(\psi \sim 0.6)$ or $\mathrm{E}_{\mathrm{c}} /\left(\sigma_{\mathrm{p}} / \mathrm{R}_{\mathrm{p}}\right)^{1 / 2}$ to be as high as possible.

Bhushan (1984, 1985a), Bhushan and Doerner (1989), Bhushan and Blackman (1991) and Oden et al. (1992) have measured the mechanical properties and surface roughnesses of varlous particulate tapes and particulate and thin-film (metal and oxide) disks. Mechanical properties of magnetic coatings of tapes are measured by dynamic mechanical analysis (DMA) system and of rigid disks were measured by a nanoindentation hardness apparatus. Surface roughness parameters of tapes and disks were measured by a noncontact optical profiler (NOP) (Bhushan et al., 1988) and an atomic force microscope (AFM) (Rugar et al., 1989). Measured values are used in the Greenwood and Williamson's contact model. The lateral resolutions for the surface topographs spanned the range of $1 \mu \mathrm{m}$ for NOP down to $2 \mathrm{~nm}$ for AFM. AFM can measure topographic features which cannot be measured with conventional profilers, Fig. 12. The NOP image of the magnetic tape in Fig. 12(a) does not show any distinctive feature. In contrast AFM image clearly shows magnetic particles. The hole in the center of disk A is the pore which is created in particulate disk which acts as reservolr for the lubricant, Fig. 12(b). The AFM has sufficiently high spatial resolution to image the grain structure of the sputtered coatings, Figs. 12(c) and 12(d). Topograph shown in Fig. 12(e) for a lapped slider shows that the surface is very smooth with grooves less than $1 \mathrm{~nm}$ deep. Bhushan and Blackman (1991) and Oden et al. (1992) found that topography and contact statistics predictions are a strong function of the lateral resolution of the roughness measurement tool, Table 4 . Here $\eta$ is the density of summits per unit area, $n$ is the number of contact spots and $\mathrm{pr}$ is the real pressure. The surface topography statistics calculated for the AFM and NOP data shows that the average summit radius $\left(R_{p}\right)$ for the $A F M$ data is two to four orders of magnitude smaller than that for the NOP data whereas summit density for the AFM data is two to four orders of magnitude larger than that for the NOP data. We note that the plasticity index $(\psi)$ calculated using the AFM data suggests that all contacts made of nanoasperities are plastic, while $\psi$ calculated with NOP data suggest that all contacts made of microasperities are elastic (see Fig. 16 to be described later).

\subsubsection{Fractal Model of Elastic-Plastic Contact}

The contact analyses developed over a last quarter century, consider only an averaged surface with a single scale of roughness to be in contact with another surface. However, due to the multiscale nature of surfaces it is found that the surface roughness parameters depend strongly on the resolution of the roughness-measuring instrument or any other form of filter, hence not unique for a surface (Bhushan et al., 1988). The dependence of variances of surface height, slope and curvature are shown in Fig. 13. The scale dependence in Fig. 13 suggests that 
Instruments with different resolutions and scan lengths yield different values of these statistical parameters for the same surface. Therefore the predictions of the contact models based on these parameters may not be unique to a pair of rough surfaces. However, if a rough surface is characterized in a way such that the structural information of roughness of all scales is retained, then it will be more logical to use such a characterization in a contact theory. In order to develop a contact theory based on this motivation, it is first necessary to quantify the multiscale nature of surface roughness.

A unique property of rough surfaces is that if a surface is repeatedly magnified, increasing detalls of roughness are observed right down to nanoscales. In addition, the roughness at all magnifications appear quite similar in structure as qualitatively shown in Fig. 14. Such a behavior can be characterized by fractal geometry (Majumdar and Bhushan, 1990, 1991b). The main conclusions from these studies were that a fractal characterization of surface roughness is scale-independent and provides information of the roughness structure at all the length scales that exhibit the fractal behavior. Based on this observation, Majumdar and Bhushan (1991a) and Bhushan and Majumdar (1991) developed a new fractal theory of contact between rough surfaces.

Consider a surface profile $\mathrm{z}(\mathrm{x})$ as shown in Fig. 14 which appears random, multiscale, and disordered. The mathematical properties of such a profile are that it is continuous, nondifferentiable and statistically self-affine. The nondifferentiability arises from the fact that a tangent or a tangent plane cannot be drawn at any point on the surface since more and more detalls of roughness will appear at the point. In short, a rough surface is never smooth at any length scale. The statistical self-affinity is due to similarity in appearance of a profile under different magnifications. It was found that the Weierstrass-Mandelbrot (W-M) function satisfies all these properties and is given as (Berry and Lewis, 1980),

$$
\begin{gathered}
z(x)=G^{(D-1)} \sum_{n=n}^{\infty} \frac{\cos \left(2 \pi \gamma^{n} x\right)}{\gamma^{(2-D) n}} ; \gamma>1,1<D<2 \\
\frac{d z}{d x} \longrightarrow \infty \text { for all } x ; z(\gamma x)=\gamma^{(2-D)} z(x)
\end{gathered}
$$

where the parameter $D$ is the fractal dimension, $G$ is a characteristic length scale of the surface and $\gamma^{n}$ are the discrete frequency modes of the surface roughness. The theory of fractal geometry and the concept of fractional dimension is well described by Mandelbrot (1982) and in the recent paper of Majumdar and Bhushan (1990). The parameters which characterize the W-M function are G, D. and $\eta_{\ell}$ where $\gamma=1.5$ was found to be sultable value for high spectral density and for phase randomization. Since a rough surface is a nonstationary random process the lowest cut off frequency is related to the length $L$ of the sample as $\eta_{\ell}=1 / L$. The parameters $G$ and $D$ can be found from the power spectrum of the $W-M$ function

$$
\mathrm{S}(\omega)=\frac{\mathrm{G}^{2(\mathrm{D}-1)}}{2 \ell \mathrm{n} \gamma} \frac{1}{\omega^{(5-2 \mathrm{D})}}
$$

where $S(\omega)$ is the power of the spectrum and $\omega$ is the frequency which is the reciprocal of the wavelength of roughness. Note that if $S(\omega)$ is plotted as a function of $\omega$ on a log-log plot, then the power law behavior would result in a straight line. The slope of the line is related to the fractal dimension D of the surface roughiess and the parameter $G$ is related to the location of the spectrum along the power axis. 
To verify whether surfaces do follow a power-1aw fractal behavior and to obtain the parameters $D$ and $G$ of a surface, one needs to compare the power spectrum of a real surface profile with that of the W-M function as given in Eq. (5). Figures 15(a) and 15(b) show the averaged spectrum of a surface profile of an untextured thin-film magnetic rigid disk of type C. The spectrum in Fig. 15(a) corresponds to the surface that was measured by an optical profiler and the spectrum in Fig. 15(b) corresponds to the surface which was measured by a scanning tunneling microscope (STM). The spectrum in Fig. 15(a) follows $S(\omega)=\omega^{-2.24}$ corresponding to $D=1.38$ whereas the spectrum in Fig. $15(\mathrm{~b})$ follows $S(\omega) \sim \omega^{-2.35}$ corresponding to $D=1.33$ and $\mathrm{G} \sim 10^{-16} \mathrm{~m}$. This data show that the surface of the rigid disk follows a fractal structure for three decades of length scales.

Bhushan et al. (1988) and Oden et al. (1992) measured the surface roughness of magnetic tape $A$ at different resolutions by NOP and AFM. Fractal analysis of the tape surface reveals two regimes of roughness demarcated by a scale of $0.1 \mu \mathrm{m}$ corresponding to the size of magnetic particles.

The fractal model of elastic-plastic contact has been developed by Majumdar and Bhushan (1991a) and Bhushan and Majumdar (1991). An interface between a statistically isotropic rough surface and a flat plane was considered. The contact spots will be of different sizes and spread randomly over the interface. Depending on the radius of curvature and height (or deformation) of the asperity, the contact spot will be either in elastic or plastic deformation. Limit of elastic deformation (propensity of yielding) is governed by the Tresca or Huber-Mises yield criteria in which the plastic flow will occur when maximum shear stress is equal to half of the tensile yield strength of the material. Whether contacts go through elastic or plastic deformation is determined by a critical area

$$
a_{c}=\frac{G^{2}}{(H / 2 E)^{2 /(D-1)}}
$$

If $\mathbf{a}<\mathbf{a}_{\mathbf{c}}$, plastic content

$$
a>a_{c} \text {, elastic content }
$$

This shows that small contact spots $\left(a<a_{c}\right)$ are in plastic contact, whereas large spots are in e lastic contact. This result is in contrast with that of the $G \&$ W model where small ones are in elastic deformation - - a prediction that is a direct implication of the assumption of uniform asperity radil. For magnetic tape $A$, typical values of the parameters are $D=1.97, G=$ $5.15 \times 10^{-9} \mathrm{~m}$ and $\mathrm{H} / \mathrm{E}=0.14$. The critical contact area for inception of plastic flow is $\mathrm{a}_{\mathrm{c}}=$ $10^{-14} \mathrm{~m}^{2}$ (contact diameter $\sim 100 \mathrm{~nm}$ ). Therefore, all contact spots larger than $100 \mathrm{~nm}$ would deform elastically. For a smooth magnetic thin-film rigid disk of type $C$, typical values of the parameters are $\mathrm{D}=1.38, \mathrm{G}=10^{-16} \mathrm{~m}$ and $\mathrm{H} / \mathrm{E}=0.06$. The critical contact area for inception of plastic deformation is $\mathrm{a}_{\mathrm{c}}=10^{-27} \mathrm{~m}^{2}$, which is practically zero. Therefore, all contact spots can be assumed to be in elastic contact at moderate loads.

The question now remains as to how do large spots become elastic when they must have been small plastic spots in their history of deformation. The possible explanation is graphically shown in Fig. 16. As two surfaces touch, the nanoasperitles (detected by AFM type of instruments) are first to come in contact. As the load is applied, the smaller asperities have smaller radil of curvature and are therefore plastically deformed instantly and the contact area increases. When the load is increased, the nanoasperities in the contact zone merge and the load is supported by elastic deformation of the larger scale asperities or microasperities (detected by NOP type of instruments). 
It is assumed that cumulative size distribution of the contact spots follow the power law relation of the form (Majumdar and Bhushan, 1991a)

$$
\mathrm{N}(\mathrm{A}>\mathrm{a})=\left(\mathrm{a}_{\ell} / \mathrm{a}\right)^{\mathrm{D} / 2}
$$

where the distribution is normalized by the area of the largest contact spot $a_{\ell}$ Since the power spectra of surface indicate that a surface can be fractal even at nanoscales, the assumption of $a_{s} \rightarrow 0$ is valid. Note that in the distribution of Eq. (7), the number of the largest spot is unity, whereas the number of spots of area $a \rightarrow 0$ would tend to infinity.

The real area of contact, $\mathrm{A}_{\mathrm{r}}$ is given as

$$
A_{r}=\frac{D}{2-D} a_{\ell} \text { for } D<2
$$

For the case $a_{\ell}>a_{c}$, the portion of the rea 1 area of contact in elastic deformation can be evaluated as

$$
A_{r e} / A_{r}=1-\left[\frac{D a_{c}}{(2-D) A_{r}}\right]^{(2-D) / 2}
$$

The total elastic-plastic load $\mathrm{W}\left(\mathrm{a}_{\ell}>\mathrm{a}_{\mathrm{c}}\right)$ is related to the real area of contact as

$$
\begin{aligned}
W / E A_{a} \sim G^{D-1} A_{r}^{D / 2}\left\{\left[\frac{(2-D) A_{r}}{D}\right]^{(3-2 D) / 2}-a_{c}^{(3-2 D) / 2}\right\} & \\
& +(H / E) A_{r}^{D / 2} a_{c}^{(2-D) / 2} \text { for } D \neq 1.5
\end{aligned}
$$

and

$$
\begin{aligned}
& W / \mathrm{EA}_{\mathrm{a}} \sim \mathrm{G}^{1 / 2} \mathrm{~A}_{\mathrm{r}}^{3 / 4} \ln \left(\mathrm{A}_{\mathrm{r}} / \mathrm{a}_{\mathrm{c}}\right) \\
& +(\mathrm{H} / \mathrm{E}) \mathrm{A}_{\mathrm{r}}^{3 / 4} \mathrm{a}_{\mathrm{c}}^{1 / 4} \text { for } \mathrm{D}=15
\end{aligned}
$$

and the total plastic load $\left(\mathrm{a}_{\ell}<\mathrm{a}_{\mathrm{c}}\right)$

$$
\mathrm{W} / \mathrm{HA}_{\mathrm{a}} \sim \mathrm{A}_{\mathrm{r}} / \mathrm{Aa}_{\mathrm{a}}
$$

We note that for the special case of $a_{c} \rightarrow 0$ (e.g., in thin-film disk $C$ ), in the elastic-plastic regime

$$
\mathrm{W} \sim \mathrm{A}_{\mathrm{r}}^{(3-\mathrm{D}) / 2}
$$

Here, the load-area relaticnshlp depends on the fractal dimension whereas G\&W predict a linear relationship. Fractal model verifies the load-area relationship observed by Bhushan (1985a) for the magnetic tape $A$ and by Bhushan and Dugger (1990a) for the thin-film disk C (Majumdar and Bhushan, 1991a). 


\subsubsection{Measurement of Contact Area}

The real area of contact of magnetic tapes and rigld disks have been measured using the optical-interference technlque by Bhushan (1985a) and Bhushan and Dugger (1990a). A loading-unloading experiment was conductcd to determine if the majority of the contacts in the measurement range ( $>0.7 \mu \mathrm{m}$ in diameter) were elastic (Bhushan, 1984). Photographs of tape contacts were taken at $28 \mathrm{kPa}$; then higher pressure (1.38 MPa) was applied for short durations and the tape contact was brought back to $28 \mathrm{kPa}$ and rephotographed, Fig. 17. There were no changes in the real area of contact after unloading to $28 \mathrm{kPa}$ which suggests that the contacts which can be detected, are elastic. This observation is in agreement with the predictions from the fractal model.

If the contacts are elasic, then the real area of contact and friction is governed by the $\mathrm{E}_{\mathrm{C}}$ and $\sigma_{\mathrm{p}} / \mathrm{R}_{\mathrm{p}}$ of the magnetic medium surface. Figure 18a shows an example that the friction of various magnetic tapes depend significantly upon the complex modulus. Stable frictional behavior was exhibited only by those tapes which displayed a complex modulus of greater than 1.2 to $1.5 \mathrm{GPa}$. Figures $18 \mathrm{~b}$ and 19 show the examples that the friction also strongly depends on the surface roughness. Typlcal contact diameters for tapes and rigid disks were found to be about $6 \mu \mathrm{m}$ and $1.5 \mu \mathrm{m}$, respectively.

Bhushan and Dugger (1990a) reported a significant increase in the contact diameter, number of contacts and total real area of contact of the thin-film rigid disk as a function of loading time, Fig. 20. Asperities under load viscoelastically and viscoplastically deform which not only increase the size of the existing asperities but also brings the two surfaces closer to allow contact of additional asperities. We expect the rate of increase in the real area of contact as a function of loading time to be dependent on the rate-dependent mechanical properties and the normal stress. Therefore, attempts should be made to select materials for disk coatings with low creep compliance, to reduce the normal stress at the head-disk interface, and to explore methods (such as load/unload mechanisms) to minimize or avold altogether the storage of head in contact with the disk. In the case of magnetic tapes, creep compliance and hydrolytic degradation characterisitcs of the binder also need to be optimized for sustained low friction after storage at high pressure (e.g., near end-of-tape on a reel) and high temperature/humidity (Bhushan, 1990).

\subsection{Liquid-Mediated Adhesion (Stiction)}

A rather smooth magnetic medium (especially thin-film disk) has a tendency to adhere or stick strongly to the smooth magnetic head. The liquid-mediated adhesion commonly referred to as "stiction" in the computer industry, is especially pronounced when liquid lubricants and adsorbed moisture arc present at the interface. Liquid-mediated adhesion can be divided into two components - a meniscus term and rate-dependent viscous term. Both components can contribute significantly to the adhesion or stiction. The meniscus term depends on the surface tension of the liquid and viscous term depends on the viscosity of the liquid. Viscous term does not depend on the surface tension and can be observed even when the surfaces are completely surrounded by the liquid. If the surfaces are submerged in the liquid they may be separated easily, provided the separation is carried out very slowly. However, if the rate of separation is rapid, the viscosity of the liquid will be the determining factor. It is easy to see that when the surfaces are pulled apart, the liquid must flow into the space between them, and if the separation is rapid, the viscosity of the liquid will be the determining factor.

For analysis purposes, we consider a model of contact region between smooth surfaces with different level of "fills" of the interface and it depends on the mean interplanar separation and the liquid levels, Fig. 21 (Matthewson and Mamin, 1988). There are two extreme regimes in which either a small quantity of liquid bridges the surfaces around the tip of a contacting asperity (the "toedipping" regime) or the liquid bridges the entire surface (the "flooded" regime); and in the third regime, the liquid bridges around from few asperities to 
large fraction of the apparent area. The different regimes can be modelled and the expressions for $F_{M}$ and FV can be obtained (Bhushan, 1990).

In the toe-dipping regime, the effect of the liquid condensate on the adhesion force between a single asperity and a surface can be modelled by a sphere of composite radius of curvature in contact with a flat surface with a liquid bridge in between. The total meniscus and viscous forces of all wetted asperity contacts can be calculated by multiplying the number of contacts by the meniscus and viscous forces at a typical contact. The flooded regime can be modelled by a liquid bridge between the two flat surfaces. The pill box regime can be modelled by two flat surfaces. If we assume that all surface asperity radil are constant and their heights follow a Gaussian distribution. the true coefficient of friction is given as follows:

For toe-dipping regime:

$$
F \sim \frac{f_{r} W}{\frac{1-16.6 \gamma_{1}\left(\cos \theta_{1}+\theta_{2}\right)}{E^{\prime} \sigma_{p}\left(\sigma_{p} / R_{p}\right)^{1 / 2}}}
$$

For flooded regine:

$$
\mathrm{F}=\mathrm{f}_{\mathrm{r}}\left[\mathrm{W}+\frac{\mathrm{A}_{\mathrm{a}} \gamma_{\ell}}{\mathrm{h}}\left(\cos \theta_{1}+\cos \theta_{2}\right]+\frac{\eta_{\ell} \mathrm{A}_{\mathrm{a}}}{\mathrm{h}}(\mathrm{L} \alpha)^{1 / 2} \mathrm{e}^{-1 / 2}\right.
$$

where

$$
\frac{h}{\sigma_{p}} \sim 1.4\left\{\log \left[0.57 \eta R_{p} \sigma_{p} E^{\prime}\left(\sigma_{p} / R_{p}\right)^{1 / 2} / P_{a}\right]\right\}^{0.65}
$$

where $\mathrm{F}$ is the friction force, $\gamma_{\ell}$ and $\eta_{\ell}$ are the surface tension and viscosity of the liquid, $\theta_{1}$ and $\theta_{2}$ are the contact angles of the liquid on the two surfaces, $h$ is the average thickness of the liquid bridge. $L$ is the distance surfaces need to slide to become unstuck, and $\alpha$ is the start-up linear acceleration. We note that the liquid-mediated adhesive forces decrease with an increase in roughness.

We make an important observation that in the toe-dipping regime, the adhesion force is independent of the apparent area and proportional to the normal load (1.e., number of asperity contacts). However, the flooded regime shows the opposite tendencies. The pillbox regime is intermediate and can exhibit either behavior at the extremes. In all three regimes, adhesion force decreases with an increase in $\sigma_{p}$ and a decrease in $R_{p}$ and is independent of $\eta$.

The relative humidity of the environment, rest period, head-slider area, surface roughness, lubricant viscosity and its thickness and relative velocity affect the liquidmediated adhesion (Liu and Mee, 1983; Bradshaw and Bhushan, 1984; Yanagisawa, 1985; Mtyoshl et al. (1988); Bhushan, 1990; Bhushan and Dugger, 1990b; Streator, 1990a, 1990b). Miyoshi et al. (1988) have measured the effect of water vapor on adhesion of a Ni-Zn ferrite pin in contact with a flat of Ni-Zn ferrite or of magnetic tape A. Fig. 22. They found that the adhesive force (normal pull-off force) of ferrtte-ferrite or ferrite-tape A contact remained low below $40 \% \mathrm{RH}$, the adhesion increased greatly with increasing relative humidity above $40 \%$. Changes in the adhesion of contacts were reversible on humidifying and dehumidifying. The adhesive forces for a liquid bridge between a spherical surface with radius same as of the pin and a flat surface were calculated using surface tension and contact angle values for water. The 
calculated values compared well with the measured values. They concluded that ferrites adhere to ferrites or tapes in a saturated atmosphere primarly from the surface tension (or meniscus effects of a thin-film of water absorbed on the interface).

Bhushan and Dugger (1990b) measured the effect of water vapor (relative humidity) and lubricant film on adhesion of a 3380-type $\mathrm{Al}_{2} \mathrm{O}_{3}$ - TiC slider in contact with a thin-film (metal) disk C. The effect of exposure time on the adhesive force at $90 \%$ RH for an unlubricated disk is shown in Fig. 23. Measurable adhesion ( $>0.1 \mathrm{mN}$ ) was observed only after 90 minutes of exposure. The adhesive force increased with the exposure time up to about 5 hours, after which there was no significant increase in adhesive force with exposure time. Effect of humidity on the lubricated and unlubricated disks is shown in Fig. 24. They found that the adhestve force (normal pull-off force) of head-disk contact remained low (below resolution of the measurement technique $\sim 50 \mathrm{mN}$ ) below $75 \% \mathrm{RH}$. the adhesion increased greatly with increasing relattve humidity above $75 \%$. The increase in the adhesive force was slightly larger for the unlubricated disk than for the lubricated one. Since the disk lubricant is hydrophobic, it repels some of the water condensation. However, water can replace some of the toplcal PFPE at concentrated asperity contacts during long exposures to water vapor or if the lubricant is squeezed out of the local contact by high pressure or displaced by sliding of the two surfaces. Studies of the penetration of lubricant layers by water (Baker et al., 1962) suggest that water may diffuse through the lubricant and condense into droplets around nuclei on the solid surfaces. Spreading of the water drop will be controlled by the energy difference between the water/disk and lubricant/disk interfaces. At this time, water with a surface tension on the order of 3 to 4 times that of typical magnetic medium lubricants, wets the magnetic-medium surface creating a meniscus at the asperity contacts. Attempting to separate the surfaces against this water film gives nse to the observed adhesion. Bhushan and Dugger also measured the adhesive force as a function of separation rate (proportional to sliding velocity), Fig. 25. They found that the adhesive force increases approximately linearly with an increase of the square root of the loading rate. This is attributed to viscous effects.

The effect of lubricant thickness and its functionality on the static and kinetic coefficients of friction in particulate disks was studied by Scarati and Caporiccio (1987), Fig. 26. We note that static friction increases with an increase in the lubricant film thickness: however, the reverse is true for kinetic friction. Increase in static friction with an increase in the lubricant thickness occurs at a lower thickness for a nonpolar lubricant than for the functional lubricant. KInetic friction decreases with an increase in the lubricant film thickness.

The effect of lubricant viscosity and its thickness, lubricant functionality, and disk surface roughness on the static and kinetic coefficients of friction on thin-iilm disks was studied by Yanaglsawa (1985) and Streator et al. (1991a). Figures 27 and 28 show the data for three disks with different roughnesses coated with four perfluoropolyether lubricants $-\mathrm{Ll}$ to $\mathrm{L} 3$ and $F$ having different film thicknesses. Jubricants $L 1, L 2$ and $L 3$ are nonpolar liquid lubricants, while $F$ is a polar liquid lubricant with dihydroxyl functional end groups. In lubricant $\mathrm{L} 3$ the carbon atoms form a branched bonding structure, while lubricants L1, L2 and $F$ contain carbon atoms in a linear arrangements. The static friction does not show a monotonic increase at the higher values of lubricant thickness as seen for kinetic friction. The coefficients of static and kinetic friction are essentially independent of lubricant thickness below a "critical" lubricant thickness. All lubricants show a sharp increase in friction at the critical thickness, which is different for each lubricant. The polar lubricant $F$ exhibits the smallest critical thickness for dramatic friction increase. In the case of nonpolar lubricants (LI, L2, and L3) the critical thickness was lower for the lower viscosity lubricants. Note that the polar lubricant $F$ does not fit within the viscosity trend exhibited among the nonpolar lubricants. The butldup of the friction is believed to be governed by micro-flow capabilities of the liquid on the disk. The lower viscosity lubricants among a class of nonpolar lubricants flow more readily to develop the meniscil bridges, resulting in higher friction than that of lubricants with higher viscosity. The polar lubricant exhibits high friction compared to the nonpolar lubricants and does not follow the viscosity trend. The increase in friction 
with increasing lubricant-film thickness above the critical thickness can be attributed to strong adhesive forces in the interface (Bhushan, 1990).

The critical thickness is correlated with disk surface roughness, which roughly corresponds to the rms roughness. The rougher surface would correspond to a larger mean separation of the surfaces, and thus a higher value of lubricant-film thickness when the meniscil are formed. The friction values above critical-film thickness are higher for smoother disks. However, these results of effect of roughness on the critical film thickness are only applicable to short contact times on the order of seconds or minutes. With longer rest times (hours or days), the adhesion usually reaches much higher value even if the lubricant thickness is well below the critical value. This time effect can be explained by the slow diffusion of the lubricant molecules towards contact points driven by the Laplace pressure and deformation of the Interacting asperities with the corresponding increase in the real area of contact.

Streator et al. (1991a) also reported that kinetic coefficient of friction decreases with increasing sliding speed.

\section{INTERFACE TEMPERATURES}

In a sliding operation, almost all of the frictional energy input is directly converted to heat in the material close to the interface. During a sliding situation, asperity interactions result in numerous high temperature flashes. Bhushan (1987a) presented a detailed thermal analysis to predict the interface temperatures and applied it to predict temperatures in a headmedium Interface (Bhushan, 1987b; 1992b). The head-medium interface can be modelled as the case of sliding two rough surfaces, Fig. 29(a). The surface proflle is measured and surface asperities are modelled with a series of spherically-topped asperities. The degree of interaction between the two surfaces at any time during sliding depends on the average contact stress. The interaction problem at an asperity contact reduces to a sphere sliding against another sphere, assuming the distance to the center of the two spheres is fixed. When one sphere comes in contact with the other, the real area of contact starts to grow; when one sphere is directly above the other, the area is at maximum; as one sphere moves away, that area starts to get smaller. Fig. 29(b). The center of the contact moves at approximately halr the relative sliding speed with respect to each asperity. The real area of contact is a source of frictional heat and the heat Intensity is proportional to the real area. Bhushan showed that the total flash temperature consists of temperature of an individual asperity contact and effect of other asperity contacts on an individual asperity temperature (interaction).

The relevant equations for the average and maximum asperity temperature rise of the interface are given as (Bhushan, 1987a)

$$
\begin{aligned}
\bar{\theta} & \left.=\mathrm{r}_{1}\left[0.65 \mathrm{fp}_{\mathrm{a}}\left(\mathrm{A}_{\mathrm{a}} / \mathrm{A}_{\mathrm{r}}\right)\left(V \mathrm{~V}_{\max } / K_{1}\right)^{1 / 2} / \rho_{1} \mathrm{Cp}_{1}+15 \mathrm{fp} \mathrm{a} \ell / K_{2}\right)^{1 / 2} / \rho_{1} \mathrm{Cp}_{1}\right]_{(14} \\
\theta_{\max } & \left.=\mathrm{r}_{1}\left[0.95 \mathrm{fp}_{\mathrm{a}}\left(\mathrm{A}_{\mathrm{a}} / \mathrm{A}_{\mathrm{r}}\right)\left(V \mathrm{~V}_{\max } / K_{1}\right)^{1 / 2} / \rho_{1} \mathrm{Cp}_{1}+15 \mathrm{fp} \mathrm{a} \ell / K_{2}\right)^{1 / 2} / \rho_{1} \mathrm{Cp}_{1}\right]_{(14 \mathrm{~b}}(\mathrm{V})
\end{aligned}
$$

and

$$
\mathrm{r}_{1} \sim 1 /\left[1+\left(\mathrm{k}_{2} \rho_{2} \mathrm{Cp}_{2} / \mathrm{k}_{1} \rho_{1} \mathrm{Cp}_{1}\right)^{1 / 2}\right]
$$

where $\rho \mathrm{Cp}$ is the volumetric heat capacity, $\mathrm{k}$ is the thermal diffusivity, $\mathrm{k}$ is the thermal conductivity, $\mathrm{d}_{\max }$ is the maximum contact diameter, $\ell$ and is the half length of the slider.

Average and maximum transient temperatures predicted for a typical particulate tapehead interface were $7^{\circ}$ and $10^{\circ} \mathrm{C}$, respectively (Bhushan, 1987b). These predictions compared 
fairly well with the infrared measurements conducted at head-tape interface by Gulino et al. (1986). The asperity-contact temperatures at head-tape interface are relatively low because of its high real area of contact, as compared to that of metal-metal or ceramic-ceramic contacts. The transient temperature of $7-10^{\circ} \mathrm{C}$ rise can lead to high friction in some tapes because the transition temperature of some tapes' mechanical properties is within $5^{\circ} \mathrm{C}$ above the ambient temperature. In isolated cases, if the magnetic particles are exposed (or get exposed in a highspeed rub) and contact the head surface, the average and maximum transient temperature rise could be about $600^{\circ} \mathrm{C}$ and $900^{\circ} \mathrm{C}$, respectively. These temperatures potentially will cause a breakdown of the medium lubricant and a degradation of the medium binder leading to excessive friction and selzure of medium motion.

Average and maximum transient temperatures predicted for a typical particulate rigiddisk-slider interface are 34 and $44^{\circ} \mathrm{C}$, respectively (Bhushan, 1992b). If the exposed magnetic particles or alumina particles contact the slider surface, the transient temperature rise could be more than $1000^{\circ} \mathrm{C}$. Average and maximum transient temperature rises for a typical thinfilm disk-slider interface are $56^{\circ}$ and $81^{\circ} \mathrm{C}$, respectively for $\mathrm{Al}_{2} \mathrm{O}_{3}$ - TiC slider and 77 and $110^{\circ} \mathrm{C}$, respectively for $\mathrm{Mn}-\mathrm{Zn}$ ferrite slider. These predictions compared fairly with the infrared measurements conducted at rigid disk-slider interface by Bair et al. (1991) and Suzukd and Kennedy (1991). The stze of an asperity contact is on the order of $1.5 \mu \mathrm{m}$ and duration of asperity contact at the full operating speed is less than $100 \mathrm{~ns}$. The thermal gradients perpendicular to the sliding surfaces are very large (a temperature drop of $90 \%$ in a depth of typically less than a contact diameter or less than a micron).

\subsection{WEAR}

\subsection{Head-(Particulate) Tab Interface}

The wear of oxide magnetic particles and ceramic head body materials is different from metallic wear because of the inherent brittleness and the relatively low surface energy of ceramics. The first sign of ferrite head wear with a magnetic tape is the appearance of very small scratches on the head surface (Fig. 30a). The physical scale of scratches is usually very fine and scratches as small as $25 \mathrm{~nm}$ have been reported (Bhushan, 1985b). Ferrite surface is microscopically removed in a brittle manner as stripes or islands, depending on the smoothness of the tape surface. Wear generally occurs by microfragmentation of the oxdde crystals in the ceramic surface. Fragmentation is the result of cleavage and transgranular fractures, one dominated by intergranular fracture. We note that the worn head surface is work hardened which reflects a shift from a mechanism dominated by transgranular fracture to one dominated by intergranular fracture. Figure $30 \mathrm{~b}$ shows a region where fracture and rupture have occurred. Such regions are commonly called "pullouts". Debris originating from these regions causes additional small scale plastic deformation and grooves (three-body abrasion). We also note that the ferrite head surface is work hardened with a large compressive stress field after wear which is detrimental to magnetic signal amplitude (Chandrasekar et al., 1987a, 1987b, 1988, 1990).

Head wear depends on the physical properties of head and medium materials, drive operating parameters, and environmental conditions. Wear data of common head materials against a $\gamma \mathrm{Fe}_{2} \mathrm{O}_{3}$ tape is shown in Fig. 31. We observe, a linear relationship between wear rate and material hardness for abrasive wear model. Head wear also depends on the grain size of the head materlal, magnetic particles, tape-surface roughness, isolated asperities on the tape surface, tape tension and tape sliding speed. Head wear as a function of surface roughness of tapes and isolated asperitles on the tape surface are shown in Figs. 32 and 33. We note that head wear increases with an increase in the surface roughness or number of isolated asperities of the tape surface. Wear rate also increases with humidity, above about $40-60 \%$ relative humidity, Fig. 34. An increase in abrasive wear at high humidities is believed to be due to moisture-assisted fracture (or static fatigue) of the grains to yield finer particles (Bhushan, 1985b; Bhushan, 1990). 
Head sliders after usage sometimes become coated with thin layers of a new organic material of high molecular weight called "friction polymers" or "tribopolymers." Friction is essentlal for the formation of these materlals. Another requirement for the formation of friction polymers in a rubbing contact is that one of the surfaces. lubricant, or even a material nearby should be organic (Lauer and Jones, 1986). It seems clear that all friction polymers are products of chemical reaction, whether they derive initially from solid polymers or from organic liquids or vapors. Friction polymers are found on head surfaces. These result in discoloration of the head surface and give an appearance of brown or blue color, and, therefore are sometimes called brown or blue stains, respectively.

During contact of particulate tape with the head in contact start/stops or during partial contact in streaming, binder and magnetic particle debris is generated primarily by adhestve wear mode. The debris can be elther loose or adherent (Bhushan and Phelan, 1986). Tape debris, loose magnetic particles, worn head material or foreign contaminants are introduced between the sliding surfaces and abrade material off each. The debris that adheres to drive components lead to polymer-polymer contact, whose friction is higher than that of rigid material-polymer contact and can lead to magnetic errors and sometimes to catastrophic fallures (Bhushan, 1990).

Calabrese et aI. (1989) conducted in situ low speed sliding experiments in which Ni-Zn ferrite pin was slid against $\mathrm{CrO}_{2}$ tape A. During wear, the tape particles consisting of binder resin and magnetic particles were generated at the contact and were literally thrown out of the interface landing within a radius of $0.8 \mathrm{~mm}$ from the pin. If the particles landed in the path of the pins, they would be drawn through the contact as the tape moved into the interface. Hence. a three-body wear scenarlo would be set up, which generated more particles. Wear particles were generally of a block-type or a flake type. The block-type particles were in the form of blocks of about $5 \mu \mathrm{m}$ and the flake type particles were typically much smaller in size.

Bhushan et al. (1986) used autoradiographic technique to conduct wear studies at headtape interface. An irradiated Ni-Zn ferrite head was run against various tapes. They found that measurable transfer of ferrite on the $\mathrm{CrO}_{2}$-tape was observed after 5,000 passes. A Co-

$\mathrm{Fe}_{2} \mathrm{O}_{3}$ tape did not show significant transfer even after 20,000 passes. Wear was represented in four distinct patterns: smeared areas, gray areas, dots or specks, and streaks or lines running in the direction of the tape. In a test, the amount of ferrite deposited on the $\mathrm{CrO}_{2}$-tape after 20,000 passes across the head was about $0.6 \mathrm{ng} / \mathrm{cm}^{2}$. In this test, about $0.6 \%$ of the generated ferrite debris was transferred to the tape, about $0.2 \%$ was transferred to the tapedrive component surfaces, and the rest was belleved to be airborne.

\subsection{Head-(Particulate) Rigid Disk Interface}

During asperity contacts, the disk debris can be generated by adhesive, abrastve and impact wear. The wear debris, generated during the manufacturing process (burnishing or buffing) of the disk, and foreign contaminants present can get trapped at the interface, which results in three-body abrasive wear, or they can get transferred to the slider, resulting in performance degradation of the alr bearing. The flash temperatures generated at asperity contacts can render any boundary lubricants ineffective and can degrade the mechanical properties of the disk binder increasing the real area of contact; this results in high friction and high disk wear. Any of these mechanisms can lead to head crash. The environment (humidity and temperature) has a significant effect on the head-disk friction and wear or debris generation.

Microscopic observations of disk and head surfaces after head crash in a CSS test show circumferential wear grooves and support elther of the two-body or three-body abrastve wear, Fig. 35. Karis et al. (1990) and Novotny et al. (1991a, 1991b) reported the complete removal of lubricant from the start/stop track at least in one region, and degradation of lubricant 
preceded the flnal stage of disk fallure. Lubrication of the disks increased the number of sliding cycles until the coating began to wear through by up to 1000 times. The number of sliding cycles until frictional fallure occurred was proportional to the areal density of lubricant.

Scarati and Caporiccio (1987) studied the effect of lubricant thickness and its functionality on the wear life of particulate media. Figure 36 shows the wear life of a particulate disk lubricated with two grades of perflunropolyether (PFPE) - Fomblin Z-25 (nonpolar) and Fomblin AM2001 (polar with reactive end groups or functional lubricant) as a function of lubricant thickness. We note that relative wear life increases with an increase in the lubricant thickness (also see Karis et al., 1990), and that polar lubricants have longer wear life than nonpolar lubricants.

The mechanism of interface fallure or head crash was studied by Kawakubo et al. (1984, 1991) for particulate disks in contact start-stop (CSS) test. Friction force, acoustic emission (AE) signal (to monitor head-disk contact), and read-back magnetic signal were measured during the test. The changes in read-back magnetic signal, friction force, and AE signal are shown in Fig. 37. At the point of interface fallure, the read-back signal decreased to almost zero and friction force and AE signal rose significantly. This implies that the head was virtually in contact even at full speed. Kawakubo et al. (1984) also videotaped the wear process through a transparent sapphire slider. They found that the disk debris transferred to the rail surfaces preceding the interface failure.

Using submicron, fluorescent polystyrene-latex particles, Hiller and Singh (1991) studied the interaction of contaminant particles with a flying slider. On the slider, the particles were deposited mainly in two regions: on the tapered region of the air-bearing rails and in the form of whiskers along the trailing ends of the ralls, Fig. 38. The whiskers contained only deformed particles, which is evidence of strong interaction between the particles and the interface, Fig. 39. After flying for sometime, large agglomerates of particles were occasionally found on the tapers. Since they contained mainly deformed particles, they could be identified to be whiskers which had detached from the trailing end. Fig. 40. No whiskers were grown on unlubricated disks, Fig. 38. This shows that liquid lubricant promotes adhesion between particles and surfaces. Liquid lubricant may thus have an adverse effect on rellabllity when large amounts of contaminant particles are present.

\subsection{Head-(Thin-Film) Rigid Disk Interface}

Magnetic films used in the construction of thin-film disks are soft and have poorer wear resistance than the particulate disks which are loaded with hard magnetic particles and load-bearing alumina particles. Thin-film disks have smoother surfaces and are designed to fly at a lower flying height than particulate rigld disks, which result in higher friction and an increased potential of head to disk interactions. During normal drive operation, the isolated asperity contacts of head and disk surfaces result in wearout of the disk surfaces by adhesive and impact wear model, and generate the debris. In addition, any asperity contacts would result in the maximum wear stress at the disk subsurface, which may initiate a crack. Repeated contacts would result in crack propagation (subsurface fatigue) leading to delamination of the overcoat and the magnetic layer. Isolated contacts in a clean environment generate very fine wear debris (primarily made of magnetic film and overcoat) which subsequently results in rather unfform disk wear from light burnishing (three-body abrasion). The disk wear results in high friction and wear in subsequent contacts, resulting in head crash (Bhushan, 1990).

External contaminations abrade the overcoat readily and result in localized damage of the disk surface by three-body abrasion. Wear debris generated at the interface invades the spacing between the head slider and the disk and/or transfers to the head slider making the head slider unstable, which leads to additional debris, and results in head crash both in the start/stop and flyabillty modes. Koka and Kumaran (1991) studied the effect of alumina 
contaminant particles on the flyabllity of a drive. A significant buildup was observed in the leading-edge taper area of the slider, and abrasive wear on the disk resulted from particles trapped in the leading-edge taper region.

Engel and Bhushan (1990) have developed a head-disk interface fallure model for thinflim disks. The principal physical variables include the sliding speed, surface topography, mechanical properties, coefficient of friction and wear rate. Surface protrusions, such as asperities and debris particles, induce impact and sliding encounters, which represent a damage rate. Fallure occurs when a specific damage rate, a characteristic for the system, is reached. Modeling uses a set of topographic parameters describing the changing, wearing surface.

\subsubsection{Role of Slider and Overcoat Materials}

Figure 41 shows the coefficient of friction as a function of number of passes for a thinfllm disk with carbon-overcoat (disk $B_{1}$ ) sliding against various slider materials (Chu et al., 1990; Chandrasekar and Bhushan, 1991). Ämongst the ceramics tested, single-crystal diamond has the lowest coefficient of friction ( 0.12) followed by partially-stabilized zirconia $(\sim 0.15)$, the remaining ceramics all have an initial coefficient of friction close to 0.2 when in contact with the disk. We note that coefliclent of friction increases with number of passes. This increase was, however, small for single crystal diamond even after it had been in contact with the disk for about 5500 passes. The rate of increase in the coefficient of friction is highest, In the case of calcium titanate and $\mathrm{Al}_{2} \mathrm{O}_{3}-\mathrm{TiC}$ sliders and $\mathrm{Mn}-\mathrm{Zn}$ ferrite and $\mathrm{ZrO}_{2}-\mathrm{Y}_{2} \mathrm{O}_{3}$ sliders exhibited a smaller increase. Calclum titanate $\left(1200 \mathrm{~kg} / \mathrm{mm}^{2}\right)$ probably showed poorest durability because it cracks readily. $\mathrm{Al}_{2} \mathrm{O}_{3}-\mathrm{TiC}$ is hardest $\left(2300 \mathrm{~kg} / \mathrm{mm}^{2}\right)$, it burnishes the disk surface more than $\mathrm{Mn}-\mathrm{Zn}$ ferrite $\left(600 \mathrm{~kg} / \mathrm{mm}^{2}\right)$ and $\mathrm{ZrO}_{2}-\mathrm{Y}_{2} \mathrm{O}_{3}\left(1300 \mathrm{~kg} / \mathrm{mm}^{2}\right)$. Examination of $\mathrm{Mn}-\mathrm{Zn}$ ferrite slider shows scratches along the air-bearing surface, which suggests that the $M n-7 n$ ferrite is slightly softer than the disk structure, and that, therefore ferrite is gentle to the disk surface. We belleve that matching of ceramic slider and disk hardnesses is essential for low wear.

An increase in overcoat hardness improves the wear resistance of the thin-film disks (Yanagisawa, 1985b). Increase in hardness of $\mathrm{SiO}_{2}$ overcoat by baking at varlous temperatures results in improvement in wear resistance as shown in Fig. 42, where the normal load was 185 $\mathrm{mN}$ and the sliding velocity was $1.12 \mathrm{~m} / \mathrm{s}$. Khan et al. (1988) have reported that hard carbon overcoats with better wear performance consist of hornogeneous grain size and uniform grain distribution across the surface and higher percentage of $\mathrm{sp}^{3}$ bonded carbon atoms (diamond structure) as compared to the carbon films with poor wear performance. Yamashita et al. (1988) reported that wear performance of unlubricated disks with $\mathrm{ZrO}_{2}-\mathrm{Y}_{2} \mathrm{O}_{3}$ overcoat is superior to that of carbon, Fig. 43. Wear performance of $\mathrm{ZrO}_{2}-\mathrm{Y}_{2} \mathrm{O}_{3}$ overcoat is comparable to carbon overcoat for lubricated disks. In a ceramic-ceramic contact, yttria-stabilized zirconia is known to have excellent friction and wear performance (Bhushan and Gupta, 1991). They also reported that $\mathrm{ZrO}_{2}$ overcoat (with $30 \mathrm{~nm}$ thickness) exhibited superior corrosion resistance than hard carbon of coated thin-film (metal) disk, when exposed to $80^{\circ} \mathrm{C} / 90 \% \mathrm{RH}$ for 7 days. Ceramic overcoats with low porosity and high electrical resistivity are known to have better electrochemical corrosion resistance (Bhushan, 1990).

Calabrese and Bhushan (1990) conducted in-situ siiding experiments of varlous head/thin-film disk (95-mm dia.) combinations in the scanning electron microscope (SEM) (also see Hedenquist et al., 1991). The purpose was to identify the initiation of particle removal during the sliding process. For example, after sliding at $50 \mathrm{~mm} / \mathrm{min}$. for a few minutes of a $\mathrm{Al}_{2} \mathrm{O}_{3}$-TiC slider on a thin-film disk with a zirconia overcoat and perfluoropolyether as the topical lubricant (disk B2), microscopic particles were removed from the edges of the rails and these particles were deposited from the head to the disk surfaces, Fig. 44. In Fig. 44, we also notice little disk debris deposited on the rall edges. Microscopic examination of the head and disk surfaces after the 20 minute test at $50 \mathrm{~mm} / \mathrm{min}$. showed that 
there was some damage to the slider edges and the disk surface was very lightly burnished with only one scratch. Minute disk debris was found on the rail edges and rall surfaces including leading taper of the head slider, Fig. 45. Continued sliding led to the increased surface change of the disk followed by catastropic failure. There appears to be several parameters that influence the initiation of particle removal. The most significant is the condition of the rail edges which contact the disk. During the start of motion between the head and the disk, the head moves with the disk until the force on the spring suspension overcomes the adhesion between the head and the disk, it will spring back in an unstable manner, causing contact with the rail edges and the disk. This could result in transfer of material from the disk or chipping of the rall edge. Calabrese and Bhushan (1990) reported head slider and disk wear to be strongly dependent on the slider and the disk overcoat materials, Table 5. Zirconia overcoat generally exhibited less wear than the carbon overcoat. $\mathrm{Mn}-\mathrm{Zn}$ ferrite slider was less aggressive to the disk than the $\mathrm{Al}_{2} \mathrm{O}_{3}$-TiC slider. The calcium titanate slider cracked early on in a sliding test, hot processing of this material appears to be a problem.

\subsubsection{Role of Lubricant Film}

The effect of lubricant viscosity and its thickness, lubricant functionality and disk surface roughness on the durabllity was studied by Miyamoto et al. (1988) and Streator et al. (1991b). The data in Fig. 46 show the friction histories of unlubricated and lubricated disks. The disk fallure is defined by the advent of a relatively sharp rise in the friction as a result of repeated sliding cycles. The effect of surface roughness is summarlzed in Fig. 47. The disk $\mathrm{X}_{2}$ demonstrates the lowest durability and indicates the effect of texturing as compared to high durability of the untextured disk, $X_{1}$. Disk $B_{1}$ is also textured, but is from a different disk manufacturer, and cannot be compared to the other disks on the basis of roughness alone. As can be seen in Fig. 47, the presence of the lubricant improved the durability of the disk over that of dry sliding in all cases. A trend exists indicating that durability increases with decreasing viscosity. The polar lubricant has significantly higher durability than the nonpolar lubricant with comparable viscosity. The greater durability of the less viscous lubricants can be attributed to their greater mobility on the disk surface. Figure 48 shows the durability results conducted on disk $\mathrm{X}_{2}$, where the lubricant film thickness is being varied. There is a general increase in durability with lubricant film thickness, as expected.

Figure 49 shows the effect of storage time on static friction for disks with a nonpolar lubricant (PFPE) and disks with dual lubricant consisting of polar (aminosilane) and nonpolar (PFPE) fraction (Hoshino et al., 1988). Increase in friction from aging the disks with dual lubricant film was found to be less than that for a disk with only nonpolar lubricant. Lubricant is also spun off with disk rotation during use. Yanagisawa (1985a) and others have shown that polar lubricants spin off less than nonpolar lubricants (Bhushan, 1990). The concept of a dual layer consisting of an unbonded layer over a bonded layer is very useful because unbonded (moblle) top layer would heal any worn areas on the disk surface where the lubricant may have been removed, and the bonded layer provides lubricant persistence.

\subsubsection{Role of Evironment}

\section{Unlubricated Disk}

Marchon et al. (1990) and Strom et al. (1991) studied the wear behavior of unlubricated thin-film disk with a carbon overcoat sliding against ceramic sliders in various environments. Strom et al. (1991) tested unlubricated carbon-coated disks (disks $B_{1}$ with no lubricant) and $\mathrm{ZrO}_{2}-\mathrm{Y}_{2} \mathrm{O}_{3}$ coated disks (disks $\mathrm{B}_{2}$ with no lubricant) in a sliding test against commercial $\mathrm{Al}_{2} \mathrm{O}_{3}$-TiC slider, Figs. $5 \mathrm{O}$ (a) and 50 (b). The average friction of carbon-coated disk increased smoothly only in the oxygen environment which indicates poor durability. also see Dimigen and Hubsch, 1983-84: Mermming et al. (1986) and Miyoshl et al. (1989). At sustalned high friction, debris was generated which reduced the real area of contact and the friction 
dropped. In the case of zirconia-coated disk, no consistent difference in the tribological behavior between various gas environments was observed.

The sliding test on carbon-coated disk was also conducted in the presence of humid gases. In this case, little difference in the friction behavior for these different gases was observed, Fig. 50(c). In all gases, the coefficient of friction increased smoothly to about 1.4 during the course of about 50 revolutions. Increase in friction in the oxygen or humid environment for the carbon-coated disk can be explained by the oxidation of the carbon film under rubbing, a tribochemical reaction. In the case of sliding on the zirconia-coated disk, no material is removed through oxidation. Wear occurs through mechanical means only, regardless of the concentration of axygen in environment.

Marchon et al. (1990) have also reported the carbon oxidation as a key process that contributes significantly to wear and friction increase with test cycles. In sliding experiments with $\mathrm{Mn}-\mathrm{Zn}$ ferrite or calcium titanate sliders and unlubricated thin-film disks with carbon overcoats, Marchon et al. (1990) reported that there is gradual increase in the coefficient of friction with repeated sliding contacts performed in air, however, in pure nitrogen, no friction increase is observed, the ccefficient of friction remaining constant at 0.2 . The altemate introduction of oxygen and nitrogen elegantly showed the role of these gases, Fig. 51 . Contact start/stop tests exhibited same effect. Marchon et al. (1990) suggested that wear process in the oxygen environment involved oxygen chemisorption on the carbon surface and a gradual loss of carbon through the formation of $\mathrm{CO} / \mathrm{CO}_{2}$ due to the action of the slider.

\section{Lubricated Disk}

Dugger et al. (1990) conducted a wear study of hemispherical pins ${ }^{*}$ (with a radius of curvature of $50 \mathrm{~mm}$ ) of $\mathrm{Mn}-\mathrm{Zn}$ ferrite sliding against a thin-film disk with carbon overcoat and perfluoropolyether as the topical lubricant (disk $B_{1}$ ) in controlled environments. They found that the contact life, as marked by the total distance slid to the point at which the coefficient of friction increases rapidly over the steady state value. is much larger in air with $50 \% \mathrm{RH}$ than in dry air or vacuum. Fig. 52(a). SEM examination of the wear scars on the pin and disk revealed morphology markedly dependent on the testing environment, Fig. 53. Characteristic of post-fallure surfaces in vacuum and dry air are severe damage to the disk surface, with the pin from the vacuum test also exhibiting extensive damage, including intergranular fracture and grain pull-out. In both cases there is also material transferred from the disk surface to the pin. In humid air, however, the contact area on the pin is covered with very fine debris (about 1 $\mu \mathrm{m})$ particles in a dark fllm (low atomic number), with fine particles on either side of the worn area. Figure 54 lllustrates the topography on the submicron scale (by STM) of the untested as well as wear track regions on disks tested to failure in vacuum and humid air. The general observation is that the surfaces of vacuum and dry air tested disks become rougher. In humid alr, on the other hand, finer grooves are seen in the surface topography, leaving a surface roughness of longer wavelength. Further surface analyses showed that the wear debris generated in humid air is much finer and is enriched with cobalt (from the magnetic layer) on its surface. In dry air and vacuum, the debris is substantially larger than one micron (Fig. 55) and tends to be enriched with nickel (probably from magnetic layer and Ni-P underlayer) on its surface. We propose that two mechanisms contribute to the observed durability differences: oxidation of metallic wear debris generated at isolated asperity contacts and alteration of the coating surface by interaction with vapor. The rate of debris oxidation depends upon the test ambient and affects the tendency for metallic debris to agglomerate through sintering or mechanical compaction into larger particles which are more damaging (Dugger et al., 1991). Significant adhesion in vacuum and less so in dry oxygen probably results in significant wear debris generation and by mechanical compaction or otherwise, small wear particles may

\footnotetext{
- Dugger et al. (1992) have reported that tests with actual slider from commercial rigid disk files yield relative contact lives that are comparable to those observed with hemispherical pins despite the apparent contact stress differences. Therefore, pins were used for acceleration in wear.
} 
agglomerate to produce large wear fragments (Fig. 55) that lead to catastrophic failure in the case of vacuum or dry air. We believe that rapid agglomeration to particle sizes of greater than a few microns is responsible for the reduction in contact life in vacuum and dry alr. However, in the $50 \% \mathrm{RH}$ alr, less adhesion at the interface and oxidation of metallic debris result in increased wear life.

Wahl et al. (1991a) and Dugger et al. (1992) further conducted tests in ultra-high-purity $(>99.999 \%)$ nitrogen, ultra-high purity ( $>99.995 \%)$ helium and $50 \% \mathrm{RH}$ nitrogen environments. Fig. 56. The contact life is short for nitrogen and helium environments and fall in the same range as the durability in vacuum. This data suggest that water vapor and oxygen in the humid air tests and oxygen in the dry air tests are responsible for the greater durabilities in these environments, while neither helium nor nitrogen plays a beneficial role for the durability of the rigid disks studied. The data for the carbon overcoat in humid nitrogen indicate that water vapor alone has a large effect on the observed improvement in durability in humid alr compared to vacuum or dry air, although both oxygen and water vapor contribute to increased durability. To explore the effects of water vapor introduction, Wahl et al. (1991a) performed durability testing using a nitrogen ambient with humidities ranging from 0.2 to $80 \%$ (these correspond to partial pressures of water vapor from about $5 \mathrm{~Pa}$ to $2600 \mathrm{~Pa}$ at room temperature), Fig. 57(a). It appears that introduction of as little as $0.2 \% \mathrm{RH}$ to the ambient dry nitrogen resulted in over two orders of magnitude increase in the contact life. Figure 57(b) shows a comparison of coefficients of friction for steady state sliding in the nitrogen amblent as a function of relative humidity. The friction is essentially independent of humidity up to a certain value of humidity and the increase in friction at very high humidity $(>80 \%)$ is believed to be due to liquid-mediated adhesion as discussed earlier, also see Dimigen and Hubsch (198384). The radical change in durability with the introduction of water vapor or oxygen is believed to be due to oxidation of wear debris in an oxidizing environment (either water vapor or oxygen) before it has a chance to agglomerate into larger and more destructive wear particles. The optimum humidity for maximum durability may depend on the stress at the interface; the interface with a small stress (e.g., small slider) may show more sensitivity to the humidity than the interface with high stress (large slider).

Dugger et al. (1992) conducted a wear study on the thin-film disk with $\mathrm{ZrO}_{2}-\mathrm{Y}_{2} \mathrm{O}_{3}$ overcoat and perfluoropolyether lubricant disk ( $\left.\mathrm{B}_{2}\right)$. Figs. 52(b) and 56. The coefficient of friction increased throughout the test ${ }^{*}$ to steady-state values between 0.6 and 1.4, depending upon the environment. Even at this high coefficient of friction, no visible wear track could be observed initially on the disk surface. When a wear track did become visible, an abrupt drop in the coefficient of friction occurred. This abrupt decrease is attributed to the generation of debris from the zirconia overcoat which, when present between the surfaces, reduced the real area of contact and hence, the friction force. The similarity of the contact lives of the zirconia overcoat in vacuum and dry air suggest that oxygen does not significantly affect the wear rate of this material. In vacuum and dry air, the disk surface exhibited extensive damage, with complete removal of the zirconla layer in some locations and transfer of metals to the pin surface. Wear particles are frequently larger than $10 \mu \mathrm{m}$. The surface morphology of the damaged area is comparable to that of carbon overcoat. In humid air, the contact life is long; the disk surface appears polished in the wear track compared to the surrounding regions, with only isolated areas of damage. The wear study on the zirconia overcoat suggest that the contact life is sensitive to the presence of water vapor.

Figure 58 shows the Auger depth profile of the unworn surface and from the wear track formed on the thin-film disk $\left(B_{1}\right)$ in humid air and stopped at $90 \%$ of the anticipated contact life (Dugger et al., 1992). These data indicate that the average carbon film thickness on the wear track is not very different from that on the untested region of the disk. Therefore, the majority of the film remains intact until very near the point of which the coefficient of friction increases dramatically above the steady-state value. Similar results have been

\footnotetext{
* In contrast, the rapid increase in coefficient of friction for carbon overcoat occurred alter significant sliding and was accompanied by a wear track on the disk visible to the naked eye.
} 
reported by Wahl et al. (1991b) for vacuum environment. Thus, the precursor to fallure is the catastrophic fallure of carbon overcoat rather than unfform thinning of the overcoat. It is believed that debris is generated at isolated points in the contact zone where the largest asperities meet. The debris accumulates until a critical debris size or volume is generated, which results in catastrophic removal of the protective carbon film.

\subsection{LUBRICATION}

Mechanical interactions between the head and the medium is minimized by the lubrication of the magnetic medium. The primary function of the lubricant is to reduce the wear of the magnetic medium and to ensure that friction remains low throughout the operation of the drive. The main challenge, though, in selecting the best candidate for a specific surface is to find a material that provides an acceptable wear protection for the entire life of the product. which can be of several years in duration. There are many requirements that a lubricant must satisfy in order to guarantee an acceptable life performance. An optimum lubricant thickness is one of these requirements. If the lubricant film is too thick, excessive stiction and mechanical failure of the head/disk is observed. On the other hand, if the film is too thin, protection of the interface is compromised and high friction and excessive wear will result in catastrophic failure. An acceptable lubricant must exhibit the properties such as chemical inertness, low volatility. high thermal, oxddative and hydrolytic stability, shear stability and good affinity to the magnetic medium surface.

Fatty acid esters are excellent boundary lubricants and the esters such as tridecyl stearate, butyl stearate, butyl palmitate, butyl myristate, stearic acid and myristic acid, are commonly used as internal lubricants roughly 1 to $3 \%$ by weight of the magnetic coating, in tapes and flexible disks. The fatty acids involved include those with acid groups with an even number between $C_{12}$ and $C_{22}$ with alcohols ranging from $C_{3}$ to $C_{13}$. These acids are all sollds with melting points above the normal surface operating temperature of the magnetic media. This suggests that the decomposition products of the ester via lubrication chemistry during a head-flexdble medium contact may be the key to lubrication.

Topkal lubrication is used to reduce the wear of rigid disks. Perfluoropolyethers (PFPEs) are chemically most stable lubricants with some boundary lubrication capability, and are most commonly used for topical lubrication of rigid disks (Bhushan, 1990). PFPEs commonly used include Fomblin $Z$ and Fomblin $Y$ lubricants made by Montiedison, Italy, Krytox $143 \mathrm{AD}$ made by Dupont. U.S.A. and Demnum made by Diakin. Japan and their difunctional derivatives containing various reactive end groups, e.g., hydroxyl (Fomblin $Z$ Dol), piperonyl (Fomblin AM 2001), and isocyanate (Fomblin Z-Disoc), all manufacturered by Montiedison. The difunctional derivatives are referred to as reactive (polar) fluoroether lubricants. The chemical structures, molecular weights and viscosities of various types of PFPE, lubricants are given in Table 6 (Cantow et al., 1986; Corti and Savelli, 1989). We note that rheological propertles of thin-films of lubricants are expected to be different from their bulk properties (Israelachvili et al., 1988; Homola et al., 1991). Fomblin $Z$ is a linear PFPE; and Fomblin $\mathrm{Y}$ and Krytox $143 \mathrm{AD}$ are branched PFPE where the regularity of the chain is perturbed by -CF3 side groups. The bulk viscosity of Fomblin $Y$ and Krytox $143 \mathrm{AD}$ is almost an order of magnitude higher than the $Z$ type. The molecular coil thickness is about $0.8 \mathrm{~nm}$ for these lubricant molecules. The monolayer thickness of these molecular depend on the molecular conformations of the polymer chain on the surface.

Fomblin $Y$ and $Z$ are most commonly used for particulate and thin-film rigid disks. Usually, lubricants with lower viscosity (such as Fomblin $Z$ types) are used in thin-film disks in order to minimize stiction. 
The local lubricant thickness is measured by Fourier transform infrared spectroscopy (FTIR), ellipsometry, angle-resolved X-ray photon spectroscopy (XPS), scanning tunneling microscopy (STM), and atomic force microscopy (AFM) (Kimachi et al., 1987; Mate et al., 1989; Bhushan, 1990; Dugger et al., 1990; Sriram et al., 1991). Ellipsometry and angle-resolved XPS have excellent vertical resolution on the order of $0.1 \mathrm{~nm}$ but lateral resolution is on the order of $1 \mu \mathrm{m}$ and $0.2 \mathrm{~mm}$, respectively. STM and AFM can measure the thickness of the liquid film with a lateral resolution on the order of their tip radius of about $100 \mathrm{~nm}$ which is not possible to achieve by other techniques.

The schematic of AFM used for measurement of localized lubricant-film thickness by Mate et al. (1989) is shown in Fig. 59. The lubricant thickness is obtained by measuring the forces on the tip as it approaches, contacts and pushes through the liquid film. In the top part of Fig. 59 is a schematic diagram of an AFM up interacting with a lubricant covered particulate disk. A typical force versus distance curve for a tungsten tip of radius $\sim 100 \mathrm{~nm}$ dipped into a disk surface coated with a perfluorinated polyether lubricant is shown in Fig. 60 . As the surface approaches the tip, the liquid wicks up causing a sharp onset of attractive force. The so-called meniscus force experienced by the tip is $\sim 4 \pi \mathrm{r} \gamma$ where $\mathrm{r}$ is the radius of the tip and $\gamma$ is the surface tension of the liquid. In Fig. 60 , the attractive force measured is about $5 \times 10^{-8}$ Newtons. While in the liquid film the forces on the lever remain constant, until repulsive contact with the disk surface occurs. The distance between the sharp snap-in at the liquid surface and the "hard-wall" of the substrate is proportional to the lubricant thickness at that point. [The measured thickness is about $2 \mathrm{~nm}$ larger than the actual thickness due to a thin layer of lubricant wetting the tip (Mate et al., 1989).] When the sample is withdrawn, the forces on the tip slowly decrease to zero as a long meniscus of liquid is drawn out from the surface.

Particulate disks were mapped by Mate et al. (1990) and Bhushan and Blackman (1991). The distribution of lubricant across an asperity was mapped by collecting force versus distance curves with the AFM in a line across the surface. Disk A was coated with nominally 20 to 30 $\mathrm{nm}$ of lubricant, but by AFM the average thickness is $2.6 \pm 1.2 \mathrm{~nm}$. (The large standard deviation reflects the huge varlation of lubricant thickness across the disk). A large percentage of the lubricant is expected to reside below the surface in the pores. In Fig. 61, we show histograms of lubricant thickness across three regions on disk $A$. The light part of the bar represents the hard wall of the substrate and the striped part on the top is the thickness of the lubricant. Each point on the histrogram is from a single force versus distance measurement separated by $25 \mathrm{~nm}$ steps. The lubricant is not evenly distributed across the surface. In regions 1 and 2 there is over twice as much lubricant than there is on the asperity (region 3). There are some points on the top and the side of the aspertty which have no lubricant coating at all (Bhushan and Blackman, 1991).

\subsection{Lubricant-Disk Surface Interactions}

The adsorption of the lubricant molecules is due to van der Waals forces, which is too weak to offset the spin off losses or to arrest displacement of the lubricant by water or other ambient contaminants. Considering that these lubricating films are on the order of a monolayer thick and are required to function satisfactorily for the duration of several years, the task of developing a workable interface is quite formidable.

An approach aiming at alleviating the above shortcomings is to enhance the attachment of the molecules to the overcoat, which, for most cases, is sputtered carbon. There are basically two approaches which have been shown to be successful in bonding the monolayer to the carbon. The first relies on exposure of the disk lubricated with neutral PFPE to various forms of radiation, such as low-energy X-ray (Heidemann and Wirth, 1984), nitrogen plasma (Homola et al., 1990) or far ultraviolet (e.g., $185 \mathrm{~nm}$ ) (Saperstein and Lin, 1990). Another approach is to use chemically-active PFPE molecule, where the various functional (reactive) end groups offer the opportunity of strong attachments to specific interface. These 
functional groups can react with respective surfaces and bond the lubricant to the disk surface which reduces its loss due to spin off and evaporation. Their main advantage, however, is their abllity to enhance durability without the problem of stiction usually assoclated with weakly bonded lubricants (Scarati and Caporiccio, 1987; Miyamoto et al., 1988; 1990; Streator et al., $1991 \mathrm{a}, 1991 \mathrm{~b})$. The effect of bonded lubricant was demonstrated elegantly in recent AFM experiments by Blackman et al. (1990). They found that when a AFM tip is brought into contact with a molecularly thin-film of a non-reactive lubricant a sudden jump into adhesive contact is observed. The adhesion was initiated by the formation of a lubricant meniscus surrounding the tip pulling the surfaces together by Laplace pressure. However, when the tip was brought into contact with a lubricant film which was firmly bonded to the surface, only a marginal adhesion, mostly due to van der Waals forces, was measured (Fig. 62).

\subsection{Lubricant Degradation}

Contacts between the slider and the lubricated disk lead to lubricant loss, Fig. 63 (Hu and Talke, 1988 and Novotny and Karis, 1991a; Novotny et al., 1991b). The lubricant polymer chain is scissioned during slider-disk contacts. The transient interface temperatures may be high enough (Bhushan. 1992b) to lead to the direct evaporation or desorption of the original lubricant molecules. Novotny and Karis (1991a) studied the difference between mechanisms by comparison of the Fomblin $Y$ and $Z$ lubricants with different chemical structure but approximately the same molecular weight:

1. In sliding, $Y$ is removed from the surface more rapidly than $Z$.

2. During flying, $Z$ is removed more rapidly from the surface than $Y$.

3. $\mathrm{Z}$ is thermally decomposed more rapidly than $\mathrm{Y}$ (Paciorek et al., 1979; Kasal et al., 1991a, 1991b).

4. Migration rates of $Z$ are faster than $Y$.

Polymer chain scission can be driven by mechanical, triboelectric, or thermal mechanisms. (Carre, 1986; Kimachl et al., 1988; Kasal et al., 199 la; Novonty and Karis, 1991a). The lubricant thickness is typically 1 to $5 \mathrm{~nm}$, and at 1 to $5 \mathrm{~m} / \mathrm{s}$ sliding velocity, the shear rate is 0.2 to $5 \times 10^{9} \mathrm{~s}^{-\mathrm{e}}$. At such high shear rates, there can be much energy imparted to the polymer chain, inducing the chains to slide over one another. If the molecules are strongly Interacting with the surface, the sliding may be hindered. Additional hindrance to interchain sliding can be the presence of a bulky side group such as the $-\mathrm{CF}_{3}$ group on the $\mathrm{Y}$ lubricant. The additional intermolecular friction of the chains with the side group can hinder the rapid configurational adjustments required to support such high shear rates. The chains can be broken by tearing bonds apart along the polymer backbone and reduced to volatile products which provide the route for the observed lubricant loss when contacts occur in sliding or flying. It also follows that the loss of $Y$ with the side group should be more rapid than that of the linear $Z$ lubricant (Novotny and Karis, 1991a).

Moreover, potential differences up to $0.1 \mathrm{~V}$ and $0.5 \mathrm{~V}$ are measured on thin-film and particulate media surfaces, respectively, between areas on and off the sliding tracks. Corresponding electric fields for slider-disk separations lead to alteration of organic materials when the localized currents pass between the disk and slider asperities (Novotny and Karis, 1991a).

Thermal decomposition of perfluoropolyethers can proceed by a free radical mechanism which involves initiation, propagation, and termination. The relative rates of initiation and propagation should be different for the two lubricants because of their chemical structure. From thermogravimetric analysis, for an equivalent rate of thermal decomposition on iron axdde, the temperature of $Y$ must be held about $100^{\circ} \mathrm{C}$ higher than that of $Z$. However, the loss of lubricant by thermal decomposition may also depend on the relative displacement 
and migration rates. Thus, the higher loss rate of $Y$ than $Z$ in sliding can also be consistent with the thermal decomposition pathway. Degradation of $Z$ lubes faster than $Y$ lubes is believed to be due to the catalytic effect of high concentration of acetal units $\left(\mathrm{CF}_{2}-\mathrm{O}\right)$ in $Z$ lubes which results in chain scission (Kasal et al., 1991a; Novotny and Karis, 1991a).

In flying, there is a displacement of lubricant to the outside of the track and a decrease of lubricant in the track. The displacement can be attributed to the repetitive application of pressure in the slider air bearing and intermittent contacts between the slider rails and the disk. Taking into account both the displacement and decrease in the lubricant level during flying, there is a net loss of lubricant from the disk surface. One possible mechanism for the loss is by aerosol droplet formation. This mechanism is reasonable given the tremendous negative pressure gradient at the tralling edge of the slider. The typical pressure increase under the air bearing rail is about $10^{5} \mathrm{~Pa}$, and this pressure drop occurs over about $10 \mu \mathrm{m}$, yielding a trailing edge pressure gradient of $10^{10} \mathrm{~Pa} / \mathrm{m}$. The rate of aerosol generation can depend on the lubricant surface tension (which is about the same for $Z$ and $Y$ ) and the lubricant molecular configuration (effectively a flow property). The $-\mathrm{CF}_{3}$ side group can act to hinder chain slippage (flow) required for efficient aerosol generation, lowering the loss rate of $Y$ below that of $Z$ in flying (Novonty and Karis, 1991a).

\section{REFERENCES}

1. Blair, S., Green, I., and Bhushan, B. (1991), "Measurements of Asperity Temperatures of a Read/Write Head Slider Bearing in Hard Magnetic Recording Disks," J. Trib., Trans. ASME (in press).

2. Baker, H. R., Bascom, W. D., and Singleterry, C. R. (1962), "The Adhesion of Ice to Lubricated Surfaces," J. Coll. Sch, 17, 447-491.

3. Berry, M. V. and Lewis, Z. V. (1980), "On the Weierstrass-Mandelbrot Fractal Function," Proc. Royal Soc., A370, pp. 459-484.

4. Bhushan, B. (1984), "Analysis of the Real Area of Contact Between a Polymeric Magnetic Medium and a Rigid Surface," J. Lub. Tech., Trans. ASME, Vol. 106, pp. 26-34.

5. Bhushan, B. (1985a), "The Real Area of Contact of Polymeric Magnetic Media-II: Experimental Data and Analysis," ASLE Trans., Vol. 28, pp. 181-197.

6. Bhushan, B. (1985b), "Assessment of Accelerated Head-Wear Test Methods and Wear Mechanisms," Tribology and Mechanics of Magnetic Storage Systems, Vol. 2 (B. Bhushan and N. S. Eiss, eds.), SP-19, pp. 101-111, ASLE, Park Ridge, Illinois.

7. Bhushan, B. (1987a), "Magnetic Head-Media Interface Temperatures, Part I-Analysis," J. Trib., Trans. ASME, Vol. 109, pp. 243-251.

8. Bhushan, B. (1987b), "Magnetic Head-Media Interface Temperatures, Part IIApplication to Magnetic Tapes," J. Trib., Trans ASME, Vol. 109, pp. 252-256.

9. Bhushan. B. (1990), Tribology and Mechanics of Magnetic Storage Devices, SpringerVerlag, New York.

10. Bhushan, B. (1992a), Mechanics and Reliability of Flexible Magnetic Media, SpringerVerlag, New York.

11. Bhushan, B. (1992b), "Magnetic Head-Media Interface temperatures, Part 3Application to Rigid Disks," $J$. Trib., Trans. ASME (in press). 
12. Bhushan, B. and Blackman, G. S. (1991), "Atomic Force Microscopy of Magnetic Rigid Disks and Sliders and its Application to Tribology." J. Trib., Trans. ASME (in press).

13. Bhushan, B., Bradshaw, R. L., and Sharma, B. S. (1984a), "Friction in Magnetic Tapes II: Role of Physical Properties," ASLE Trans., Vol. 27, pp. 89-100.

14. Bhushan, B. and Doerner, M. F. (1989), "Role of Mechanical Properties and Surface Texture in the Real Area of Contact of Magnetic Rigid Disks," J. Trib., Trans. ASME, Vol. 111, pp. 452-458.

15. Bhushan, B. and Dugger, M. T. (1990a), "Real Contact Area Measurements on Magnetic Rigid Disks," Wear, Vol. 137, pp. 41-50.

16. Bhushan, B. and Dugger M. T. (1990b), "Liquid-Mediated Adhesion Measurements at the Thin-Film Magnetic Disk/Head Slider Interface," J. Trib., Trans. ASME, Vol. 112, pp. 217-223.

17. Bhushan, B. and Gupta, B. K. (1991), Handbook of Tribology: Materials Coatings, and Surface Treatments, McGraw-Hill, New York.

18. Bhushan, B. and Majumdar, A. (1991), "Elastic-Plastic Contact Model of Bifractal Surfaces," Wear (in press).

19. Bhushan, B., Nelson, G. W., and Wacks, M. E. (1986). "Head-Wear Measurements by Autoradiography of the Worn Magnetic Tapes," J. Trib., Trans. ASME, Vol. 108, pp. 241-255.

20. Bhushan, B. and Phelan, R. M. (1986). "Frictional Properties as a Function of Physical and Chemical Changes in Magnetic Tapes During Wear," ASLE Trans., Vol. 20, pp. 402413.

21. Bhushan, B., Sharma, B. S., and Bradshaw, R. L. (1984b), "Friction in Magnetic Tapes I: Assessment of Relevant Theory," ASLE Trans., Vol 27, pp. 33-44.

22. Bhushan, B., Wyant, J. C., and Meiling. J. (1988), “A New Three-Dimensional Digital Optical Profller," Wear, Vol. 122, pp. 301-312.

23. Blackman, G. S., Mate, C. M., and Philpott, M. R. (1990), "Interaction Forces of a Sharp Tungsten Tip with Molecular Films on Silicon Substrate," Phys. Rev. Lett., Vol. 65, pp. 2270-2273.

24. Bowden, F. P. and Tabor, D. (1950), Friction and Lubrication of Solids, Part I, Clarendon Press, Oxford, U. K.

25. Bradshaw, R. L. and Bhushan, B. (1984), "Friction in Magnetic Tapes Part III: Role of Chemical Properties," ASLE Trans., Vol. 27, pp. 207-219.

26. Bradshaw, R. L. and Bhushan, B., Kalthoff, C., and Warne, M. (1986), "Chemical and Mechanical Performance of Flexible Magnetic Media Contalning Chromium Dioxide," IBM J. Res. Develop., Vol. 30, pp. 203-216.

27. Calabrese, S. J., Bhushan, B., and Davis, R. E. (1989), "A Study of Scanning Electron Microscopy of Magnetic Head-Tape Interface Sliding," Wear, Vol. 131, pp. 123-133.

28. Calabrese, S. J., Bhushan, B. (1990), "A Study of Scanning Electron Microscopy of Magnetic Head-Disk Interface Sliding," Wear, Vol. 139, pp. 367-381.

29. Camras, M. (1988), Magnetic Recording Handbook, Van Nostrand Reinhold, New York. 
30. Cantow, M. J. R., Larrabee, R. B., Barrall, E. M., Butner, R. S., Cotts, P., Levy, F., and Ting, T. Y., (1986), "Molecular Welghts and Molecular Dimensions of

Perfluoropolyether Fluids," Makromol. Chem. Vol. 187, pp. 2475-2481.

31. Carre, D. J. (1986), "Perfluoropolyether Oil Degradation-Inference of FeF3 Formation on Steel Surfaces Under Boundary Lubrication," ASLE Trans., Vol. 29, pp. 121-125.

32. Chandrasekar, S., Shaw, M. C., and Bhushan, B. (1987a), “Comparison of Grinding and Lapping of Ferrites and Metals," J. Eng. for Indus., Trans. ASME, Vol. 109, pp. 76-82.

33. Chandrasekar, S., Shaw, M. C., and Bhushan, B. (1987b), "Morphology of Ground and Lapped Surfaces of Ferrite and Metal," J. Eng. for Indus., Trans. ASME, Vol. 109, pp. 8386.

34 Chandrasekar, S. and Bhushan, B. (1988), "Control of Surface Finishing Residual Stresses in Magnetic Recording Head Materials," J. Eng. Jor Indus., Trans. ASME. Vol. 110. pp. 87-92.

35. Chandrasekar, S., Kokini, K., and Bhushan, B. (1990), "Influence of Abrasive Properties on Residual Stresses in Lapped Ferrite and Alumina," J. Amer. Ceramic Soc., Vol. 73, pp. 1907-1911.

36. Chandrasekar, S. and Bhushan, B. (1991), "Friction and Wear of Ceramics for Magnetic Recording Applications-Part II: Friction Measurements," J. Trib., Trans. ASME, Vol. 113. pp. 313-317.

37. Chu, M. Y., De Jonghe, L., and Bhushan, B. (1990). "Wear Behavior of Ceramic Sliders in Sliding Contact with Rigid Magnetic Thin-Film Disks," Tribology and Mechanics of Magnetic Storage Systems (B. Bhushan. Ed.), Vol. 7, pp. 9-16, STLE, Park Rldge, Illinols.

38. Corti, C. and Savelli, P. (1989), "Perlluoropolyether Lubricants: Physical and Tribology Performances and Applications." Proc. 5th Int. Congress on Tribology (K. Holmberg and I. Nieminen, eds.), Vol. 5, pp. 155-164, Finnish Society for Tribology, Helsinki, Finland.

39. Dimigen, H. and Hubsch, H. (1983-84), "Applying Low-Friction Wear-Resistant Thin Solid Films by Physical Vapour Deposition." Philips Tech. Rev., Vol. 41, pp. 186-197.

40. Doan, T. Q., and Mackintosh, N. D. (1988), "The Frictional Behaviour of Rigid-Disk Carbon Overcoats," Tribology and Mechanics of Magnetic Storage Systems, Vol. 5, (B. Bhushan, and N. S. Eiss, eds.), SP-25, pp. 6-11, STLE, Park Ridge, Illinols.

41. Dugger, M. T., Chung, Y. W., Bhushan, B., and Rothschild, W. (1990), "Friction, Wear, and Interfacial Chemistry in Thin-Film Magnetic Rigid Disk Files," J. Trib., Trans. ASME. Vol. 112, pp. 238-245.

42. Dugger, M. T., Wahl, K. J., Chung, Y. W., Bhushan, B., and Rothschild, W. (1991), “An Investigation of Environmental Effects on the Wear and Surface Composition of ThinFilm Magnetic Disks," Advances in Engineering Tribology, (Y. W. Chung and H. S. Cheng, eds.), STLE, Park Rddge, lllinois (in press).

43. Dugger, M. T., Chung, Y. W., Bhushan, B., and Rothschild, W. (1992), "Wear Mechanisms of Amorphous Carbon and Zirconla Coatings on Rigid Disk Magnetic Recording Media," Tribology Trans. (in press).

44. Engel, P. A. and Bhushan, B. (1990), "Sliding Fallure Model for Magnetic Head-Disk Interface," J. Trib.. Trans. ASME, Vol. 112, pp. 299-303. 
45. Greenwood, J. A. and Wlllamson, J. B. P. (1966), "Contact of Nominally Flat Surfaces," Proc. Roy. Soc. (Lond.). Vol. A295. pp. 300-319.

46. Gulino, R., Balr, S., Winer, W. O., and Bhushan, B. (1986). "Temperature Measurement of Microscopic Areas within a Simulated Head/Tape Interface Using Infrared Radiometric Technique," J. Trib., Trans. ASME, Vol. 108, pp. 29-34.

47. Hahn, F. W. (1984), "Head Wear as a Function of Isolated Asperities on the Surface of Magnetlc Tape," IEEE Trans. on Magn., Vol, Mag-20, pp. 918-920.

48. Hedenquist, P., Olsson, M., Hogmark, S. and Bhushan, B. (1991), "Triboiogical Studies of Varlous Magnetic Heads and Thin-Film Rigid Disks," Wear (in press).

49. Heidemann, R. and Wirth, M. (1984), "Transforming the Lubricant on a Magnetic Disk into a Solid Fluorine Compound," IBM Tech. Disclosure Bull., Vol. 27, pp. 3199-3205.

50. Hiller, B, and Singh, G. P. (1991), "Interaction of Contaminant Particles with the Particular Slider/Disk Interface," Adv. Info. Storage Syst., Vol. 2, pp. 173-180.

51. Hoagland, A. S. (1963), Digital Magnetic Recording, Wiley, New York.

52. Homola, A. M., Lin, L. J., and Saperstein, D. D. (1990), "Process for Bonding Lubricant to a Thin-Film Magnetic Recording Disk," U. S. Patent 4, 960, 609, Oct. 2.

53. Homola, A. M., Nguyen. H. V., and Hadziloannous, G. (1991), "Influence of Monomer Architecture on the Shear Froperties of Molecularly Thin Polymer Melts." J. Chem. Phys. (in press).

54. Hoshino, M., Kimachi, Y., Yoshimura, F., and Terada, A. (1988), "Lubrication Layer Using Perfluoropolyether and Aminosilane for Magnetic Recording Media." Tribology and Mechantcs of Magnetic Storage Systems, Vol. 5 (B. Bhushan and N. W. Eiss, eds.). SP-25, pp. 37-42, STLE, Park Ridge, Illinois.

55. Hu, Y, and Talke, F. E. (1988), "A Study of Lubricant Loss in the Rail Region of a Magnetic Recording Slider Using Ellipsometry." Tribology and Mechanics of Magnetic Storage Systems, Vol. 5 (B. Bhushan and N. W. Eiss, eds.), SP-25, pp. 43-48, STLE, Park Ridge, Illinois.

56. Israelachvili, J. N., McGuiggan, P. M., and Homola, A. M. (1988), "Dynamic Properties of Molecularly Thin Liquid Films," Science, Vol. 240, pp. 189-191.

57. Iwasakd, S. and Nakamura, Y. (1977). "An Analysis for the Magnetization Mode for High Density Magnetic Recording," IEEE Trans. Magn., Vol. Mag-13, pp. 1272-1277.

58. Jorgensen, F. (1988), The Complete Handbook of Magnetic Recording. Third ed., Tab Books Inc., Blue Ridge Summit, Pennsylvania.

59. Karis, T. E., Novotny, V. J., and Crone, R. M. (1990), "Sliding Wear Mechanism of Particulate Magnetic Recording Media," Tribology and Mechanics of Magnetic Storage Systems, Vol. 7 (B. Bhushan, ed.), SP-29, pp. 35-42, STLE, Park Ridge, Illinois.

60. Kasal, P. H., Tang. W. T., and Wheeler, P. (1991), "Degradation of Perfluoropolyethers Catalyzed by Aluminlum Oxide," Appl. Surf. Sci. (in press).

61. Khan, M. R., Heiman, N., Fisher, R. D., Smith, S., Smallen, M., Hughes, G. F., Veirs, K., Marchon, B., Ogletree, D. F., Salmeron, M., and Siekhaus, W. (1988), "Carbon Overcoat 
and the Process Dependence on its Microstructure and Wear Characteristics," IEEE Trans. on Magn., Vol. 24, pp. 2647-2649.

62. Kimachi, Y., Yoshimura, F., Hoshino, M., and Terada, A. (1987), "Uniformity Quantification of Lubricant Layer on Magnetic Recording Media," IEEE Trans. Magn., Vol. Mag-23, pp. 2392-2394.

63. Koka, R. and Kumaran, A. R. (1991), "Visualization and Analysis of Particulate Buldup on the Leading Edge Tapers of Sliders," Adv. Info. Storage Syst., Vol. 2, pp. 161-171.

64. Lauer, J. L. and Jones, W. R. (1986). "Friction Polymers," Tribology and Mechanics of Magnetic Storage Systems, Vol. 3 (B. Bhushan, and N. S. Eiss, eds.), SP-21, pp. 14-23, ASLE, Park Ridge, Illinois.

65. Liu, C. C. and Mee, P. B. (1983), "Stiction at the Winchester Head-Disk Interface." IEEE Trans. Magn., Vol. Mag-19, pp. 1659-1661.

66. Lowman, C. E. (1972), Magnetic Recording, McGraw Hill, New York.

67. Majumdar, A. and Bhushan, B. (1990), "Role of Fractal Geometry in Roughness Characterization and Contact Mechanics of Surfaces," J. Trib., Trans. ASME, Vol. 112, pp. 205-216.

68. Majumdar, A. and Bhushan, B. (1991a), "Fractal Modei of Elastic-Plastic Contact Between Rough Surfaces," J. Trib., Trans. ASME, Vol. 113, pp. 1-I1.

69. Majumdar, A., Bhushan, B., and Tien, C. L. (1991b), "Role of Fractal Geometry in Tribology," Adv. Info. Storage Syst., Vol. 1, pp. 231-266.

70. Mandelbrot, B. B. (1982), The Fractal Geometry of Nature, W. H. Freeman, New York.

71. Marchon, B., Heiman. N., and Khan, M. R. (1990), "Evidence for Tribochemical Wear on Amorphous Carbon Thin Films," IEEE Trans. Magn.. Vol. 26, pp. 168-170.

72. Mate, C. M., Lorenz, M. R., and Novotny, V. J. (1989), "Atomic Force Microscopy of Polymeric Liquid Films," J. Chem. Phys., Vol. 90, pp. 7550-7555.

73. Mate, C. M., Lorenz, M. R., and Novotny, V. J. (1990), "Determination of Lubricant Film Thickness on a Particulate Disk by Atomic Force Microscopy," IEEE Trans. Magn., Vol. Mag-26, pp. 1225-1228.

74. Matthewson, M. J. and Mamin, H. J. (1988), "Liquid-Mediated Adhesion of Ultra-Flat Solid Surfaces," Proc. Mat. Res. Soc. Symp., Vol 119, pp. 87-92.

75. Mee, C. D. and Daniel, E. D. eds. (1990), Magnetic Recording Handbook, McGraw Hill, New York.

76. Memming, R., Tolle, H. J., and Wierenga, P. E. (1986), "Properties of Polymeric Layers of Hydrogenated Amorphous Carbon Produced by a Plasma-Activated Chemical Vapor Deposition Process II: Tribological and Mechanical Properties," Thin Solid Films, Vol. 143, pp. 31-41.

77. Mlyamoto, T., Sato, I., and Ando, Y. (1988), "Friction and Wear Characteristics of ThinFilm Disk Media in Boundary Lubrication," Tribology and Mechanics of Magnetic Storage Systems, Vol. 5 (B. Bhushan, and N. S. Eiss, eds.), SP-25, pp. 55-61, STLE, Park Ridge, Illinois. 
78. Miyamoto, T., Sato, I., and Ando, Y. (1990), "Interaction Force Between Thin-Film Disk Media and Elastic Solids Investigated by Atomic Force Microscopy," J. Trib., Trans. ASME, Vol. 112, pp. 567-572.

79. Młyoshi, K., Buckley, D. H., Kusaka, T., Maeda, C., and Bhushan. B. (1988), "Effect of Water Vapor on Adhesion of Ceramic Oxide in Contact with Polymeric Magnetic Medium and Itself," Tribology and Mechanics of Magnetic Storage Systems, Vol. 5 (B. Bhushan, and N. S. Eiss, eds.). SP-25, pp. 12-16. STLE, Park Rddge, Mlinois.

80. Miyoshi, K., Pouch, J. J., and Alterovitz, S. A. (1989), "Plasma-Deposited Amorphous Hydrogenated Carbon Films and Their Tribological Properties," Tech. Memo 102379. NASA Lewis Research Center, Cleveland, Ohlo.

81. Nishihara, H. S., Dorius, L. K., Bolasna, S. A., and Best, G. L. (1988), "Performance Characteristics of IBM 3380K Air Bearing Design," Tribology and Mechanics of Magnettc Storage Systems, Vol. 5 (B. Bhushan, and N. S. Eiss, eds.), SP-25, pp. 117-123, STLE, Park Ridge, Illinols.

82. Novotny, V. J. and Karis, T. E. (1991a), "Sensitive Tribological Studies on Magnetic Recording Disks," Adv. Info. Storage Syst., Vol. 2, pp. 137-152.

83. Novotny, V. J., Karis, T. E., and Johnson, N. W. (1991b), “Lubricant Removal, Degradation, and Recovery on Particulate Magnetic Recording Media," J. Trib., Trans. ASME (in press).

84. Oden, P. I., Majumdar, A., Bhushan, B., Padmanabhan, A., and Graham, J. J. (1992), "AFM Imaging, Roughness Analysis and Contact Mechanics of Magnetic Tape and Head Surfaces," J. Trib., Trans. ASME (in press).

85. Paciorek, K. J. L., Kratzer, R. H., Kaufman, J., and Nakahara, J. H. (1979), "Thermal Oxidative Studies of Poly (hexafluoropropene oxide) Fluids," J. Appl. Poly. Sci, Vol. 24, pp. 1397-1411.

86. Rugar, D., Mamin, H. J., and Guethner, P. (1989), "Improved Fiber Optic Interferometer for Atomic Force Microscopy," Appl. Phys. Lett., Vol. 55, pp. 2588-2590.

87. Saperstein, D. D. and Lin, L. J. (1990), "Improved Surface Adhesion and Coverage of Perfluoropolyeter Lubricants Following Far-UV Irradiation," Langmuir, Vol. 6, pp. 1522-1524.

88. Scarati, A. M. and Caporiccio, G. (1987), "Frictional Behavior and Wear Resistance of Rigid Disks Lubricated with Neutral and Functional Perfluoropolyethers," IEEE Trans. Magn., Vol. Mag-23, pp. 106-108.

89. Sriram, T. S., Wahl, K. J., Chung, Y. W., Bhushan, B., and Rothschild, W. (1991), "The Application of Scanning Tunneling Microscopy to Study Lubricant Distribution of Magnetic Thin-Film Rigid Disk Surfaces," J. Trib.. Trans. ASME, Vol. 113, pp. 245-248.

90. Streator, J. L., Bhushan, B., and Bogy, D. B. (1991a), "Lubricant Performance in Magnetic Thin-Film Disks with Carbon Overcoat-Part I: Dynamic and Static Friction," J. Trib., Trans. ASME, Vol. 113, pp. 22-31.

91. Streator, J. L., Bhushan, B., and Bogy, D. B. (1991b), "Liquid Performance in Magnetic Thin-Film Disks with Carbon Overcoat-Part II: Durability, ${ }^{~ J}$. Trib., Trans. ASME, Vol. 113, pp. 32-37. 
92. Strom, B. D., Bogy, D. B., Bhatia, C. S., and Bhushan, B. (1991), Tribochemical Effects of Various Gases and Water Vapor on Thin-Film Magnetic Disks with Carbon Overcoats," J. Trlb., Trans. ASME (In press).

93. Suzukd, S. and Kennedy, F. E. (1991), "The Detection of Flash Temperatures in a Sliding Contact by the method of Tribo-Induced Thermoluminescence," $J$. Trib., Trans. ASME, Vol. 113, pp. 120-127.

94. Tanaka, K. and Miyazakı, O. (1981), "Wear of Magnetic Materials and Audio Heads Sliding Against Magnetic Tapes," Wear, Vol. 66, pp. 289-306.

95 Van Gestel, W. J., Gorter, F. W., and Kutjk, K. E. (1977), "Read-out of a Magnetic Tape by the Magnetoresistive Effect," Philips Tech. Rev., Vol. 37, No. 2/3, pp. 42-50.

96. Wahl, K. J., Chung, Y. W., Bhushan, B., and Rothschild, W. J. (1991a), "Durability of Magnetic Thin-Film Rigid Disks in Nitrogen and Helium Environments," Adv. Info. Storage Syst., Vol. 1, pp. 327-336.

97. Wahl, K. J., Chung, Y. W., Bhushan, B., and Rothschild, W. J. (1991b), "In Situ Auger Measurements of Surface Chemical Changes of Magnetic Thin-Film Rigid Disks During Spherical Pin Sliding Tests," Adv. Info. Storage Syst., Vol. 3 (in press).

98. Wallace, R. L. (1951), "The Reproduction of Magnetically Recorded Signal," Bell Syst. Tech J., Vol. 30, pp. 1145-1173.

99. Yamashita, T., Chen, G. L., Shir, J., and Chen, T. (1988), "Sputtered $\mathrm{ZrO}_{2}$ Overcoat with Superior Corrosion Protection and Mechanical Performance in Thin-Film Rigid Disk Application," IEEE Trans. Magn., Vol. Mag-24, pp. 2629-2634.

100. Yanagisawa, M. (1985a), "Lubricants on Plated Magnetic Recording Disks," Tribology and Mechanics of Magnetic Storage Systems, Vol. 2 (B. Bhushan, and N. S. Eiss, eds.), SP-19, pp. 7-15, ASLE, Park Ridge, Illinois.

101. Yanagisawa, M. (1985b), "Tribological Properties of Spin-Coated $\mathrm{SiO}_{2}$ Protective Film on Plated Magnetic Recording Disks," Tribology and Mechanics of Magnetic Storage Systems, Vol. 2 (B. Bhushan, and N. S. Eiss, eds.), SP-19, pp. 16-20, ASLE, Park Ridge, Illinois. 
Table 1. Selected physical properties of hard head materials

\begin{tabular}{|c|c|c|c|c|c|}
\hline Material & $\begin{array}{l}\text { Density, } \\
\mathrm{kg} / \mathrm{m}^{3}\end{array}$ & $\begin{array}{l}\text { Young's } \\
\text { Modulus, } \\
\text { GPa }\end{array}$ & $\begin{array}{c}\text { Knoop } \\
\text { microhardness, } \\
\mathrm{GPa}\left(\mathrm{kg} / \mathrm{mm}^{2}\right) \\
\end{array}$ & $\begin{array}{c}\text { Flexural } \\
\text { strength, } \\
\mathrm{MPa} \\
\end{array}$ & $\begin{array}{l}\text { Electrical } \\
\text { resistivity, } \\
\mu o \mathrm{hm} \cdot \mathrm{cm}\end{array}$ \\
\hline Ni-Zn ferrite & 4570 & 122 & $6.9(700)$ & 150 & $10^{11}$ to $10^{13}$ \\
\hline Mn-Zn ferrite & 4570 & 122 & $5.9(600)$ & 120 & $5 \times 10^{4}$ to $5 \times 10^{5}$ \\
\hline $\mathrm{Al}_{2} \mathrm{O}_{3}-\mathrm{TiC}(70-30)$ & 4220 & 450 & $22.6(2300)$ & 880 & $2 \times 10^{3}$ to $3 \times 10^{3}$ \\
\hline $\mathrm{ZrO}_{2}-\mathrm{Y}_{2} \mathrm{O}_{3}(94-6)$ & 6360 & 210 & $12.8(1300)$ & $500-700$ & $10^{16}$ \\
\hline $\mathrm{BaTiO}_{3}$ & 4320 & 110 & $10.3(1050)$ & & \\
\hline
\end{tabular}




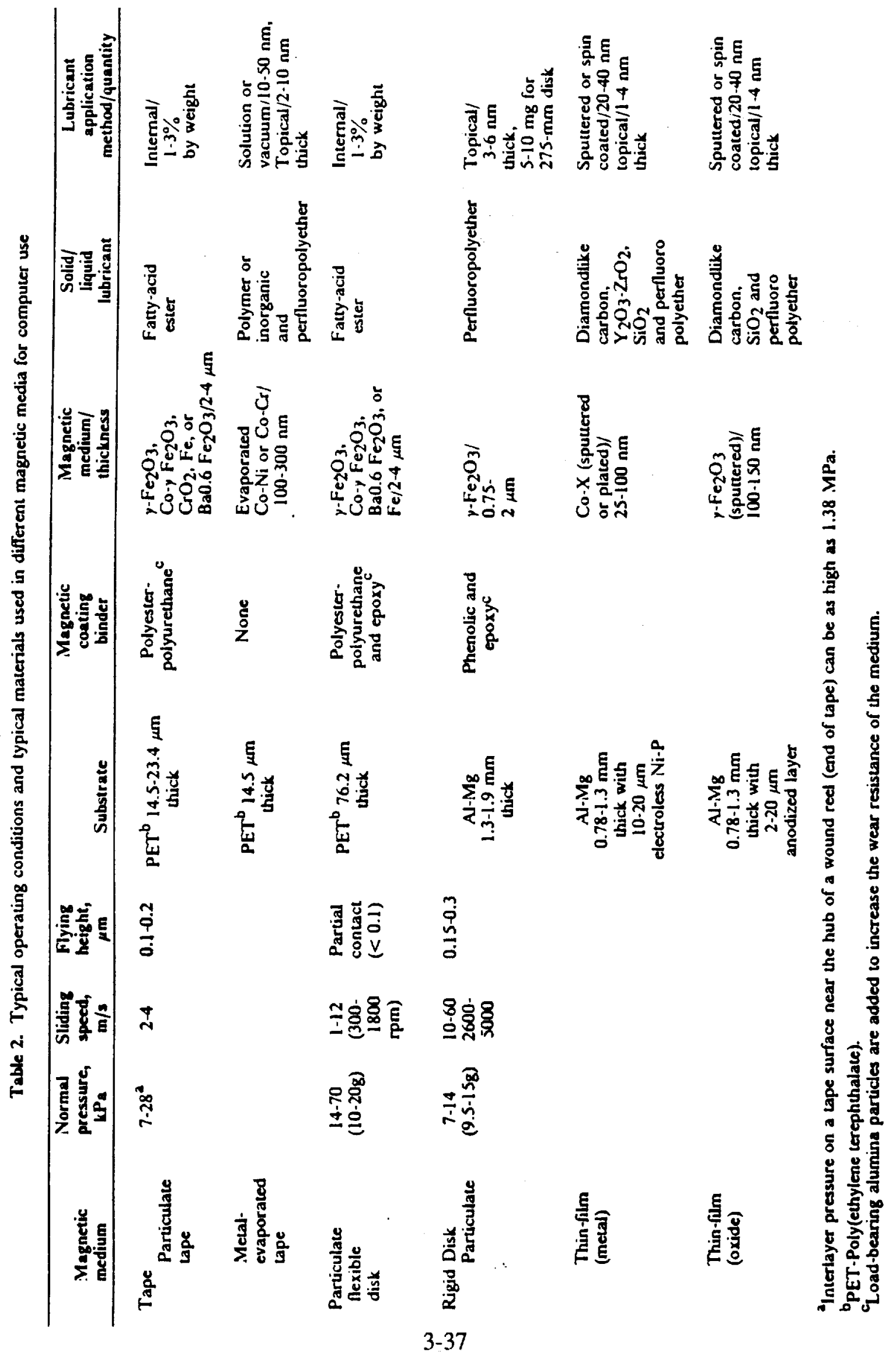


Table 3. Selected physical properties of magnetic media and its components

\begin{tabular}{|c|c|c|c|}
\hline Material & $\begin{array}{l}\text { Density, } \\
\mathrm{kg} / \mathrm{m}^{3}\end{array}$ & $\begin{array}{c}\text { Young's } \\
\text { modulus, } \\
\text { GPa } \\
\end{array}$ & $\begin{array}{c}\text { Knoop } \\
\text { microhardness, } \\
\mathrm{GPa}\left(\mathrm{kg} / \mathrm{mm}^{2}\right) \\
\end{array}$ \\
\hline$\gamma-\mathrm{Fe}_{2} \mathrm{O}_{3}$ particles & 4800 & - & $11.8(1200)$ एst. \\
\hline $\begin{array}{l}\text { Magnetic flexible medium } \\
\text { substrate }\end{array}$ & 1.520 & $2.75-4.50$ & $0.20(20)$ \\
\hline $\begin{array}{l}\text { Particulate flexible medium } \\
\text { coating }\end{array}$ & 1660 & $1.25-2.25$ & $0.25(25)$ \\
\hline$\Lambda \mathrm{l}-\mathrm{Mg}(96-4)$ & 2700 & 70 & $0.88(90)$ \\
\hline Particulate rigid disk coating & 1810 & 10 & $0.50(51)$ \\
\hline Ni-P (electroless plated) & 8910 & 130 & $5.9-7.9(600-800)$ \\
\hline $\mathrm{CrO}_{3}$ anodized layer (alumite) & - & - & $3-5(310-510)$ \\
\hline $\begin{array}{l}\text { Chemically strengthened alkali- } \\
\text { aluminosilicate glass }\end{array}$ & 2460 & 73 & $5.8(590)$ \\
\hline $\begin{array}{l}\text { Sputtered Co-Cr alloy metal } \\
\text { film }\end{array}$ & 8030 & $170-210$ & $6.9-8.8(700-900)$ \\
\hline $\begin{array}{l}\text { Diamondlike carbon overcoat } \\
\text { for metal film }\end{array}$ & 2100 & $160-180$ & $14.7-19.6(1500-2000)$ \\
\hline Thin-film rigid disk structure & - & 110.140 & $6-10)(610-1020)$ \\
\hline
\end{tabular}




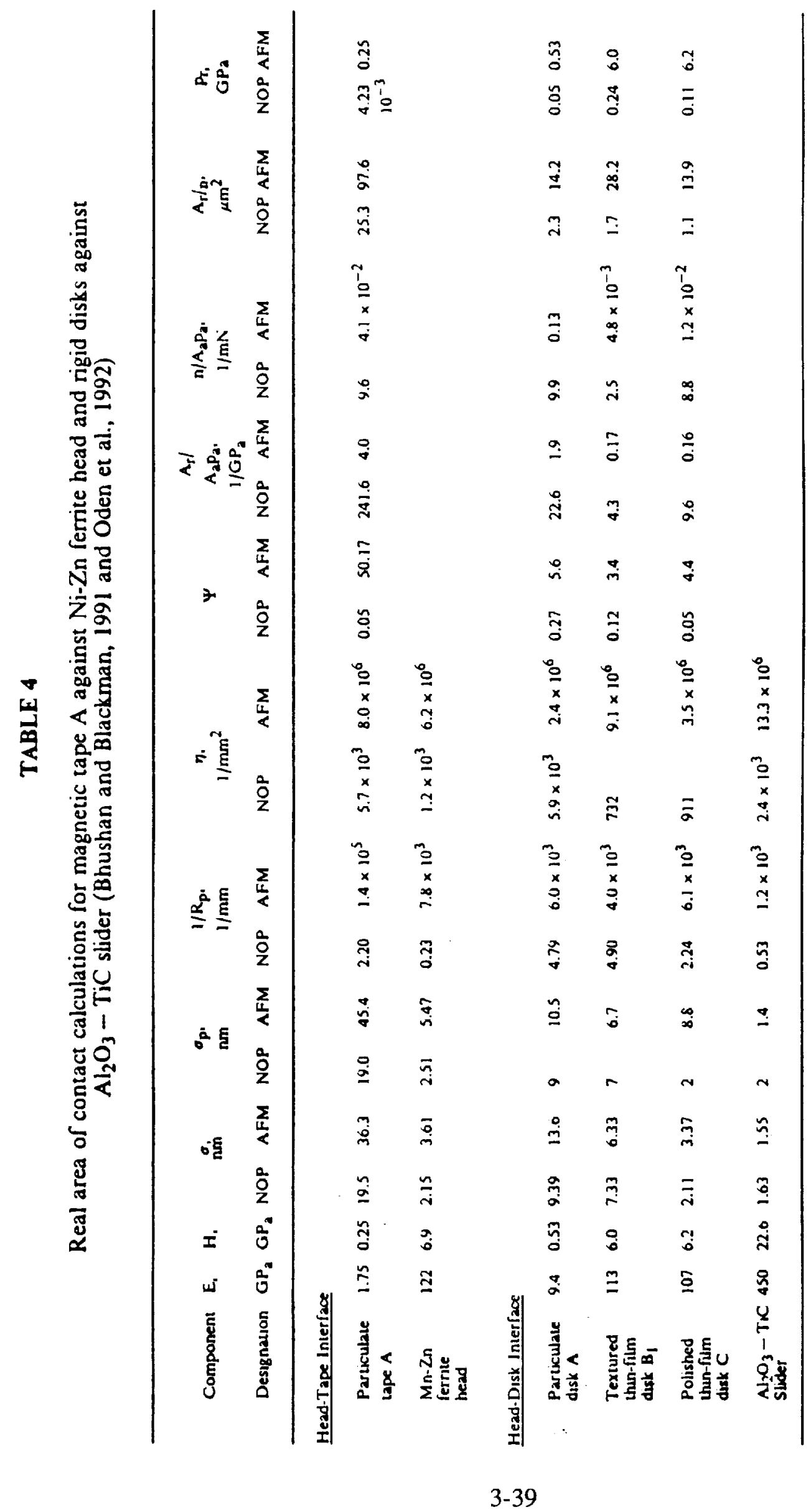




\section{Table 5}

Ranking of thin-film rigid disk and slider material combinations identified by in situ teting in the SEM (Calabrese and Bhushan, 1990)

$\mathrm{Mn}-\mathrm{Zn}$ ferrite slider vs. disk $\mathrm{B}_{2}\left(7 . \mathrm{rO}_{2}-\mathrm{Y}_{2} \mathrm{O}_{3}\right)$ Some transfer to the edges. I.ow breakaway friction value. No damage to the disk surface. !arge grain size particles do not appear to contribute to wear or damage during subsequent sliding. Rated best

$\mathrm{Al}_{2} \mathrm{O}_{3}$-TiC slider vs. disk $\mathrm{B}_{2}\left(7 . \mathrm{rO}_{2}-\mathrm{Y}_{2} \mathrm{O}_{3}\right)$ Transfer to the rail cdges. Fine grain wear debris gets trapped at the interface and contributes to damage during subsequent sliding. Rated good

Mn-Zn ferrite slider vs. disk $B_{1}$ (DI,Ca)

$\mathrm{NI}_{2} \mathrm{O}_{3}$-TiC slider vs. disk $\mathrm{B}_{1}$ (DI.C)

Calcium titanate slider vs. disk $B_{1}$ (DI.C) Transfer to the rails. No damage to the disk but disk is wearing. Rated fair Transfer to the rails. Damage to the disk on breakaway. Rated fair.

Severe damage to the disk, heavy disk wear. llead slider appears to be cracking. Rated poor

aDLC - diamondlike carbon 


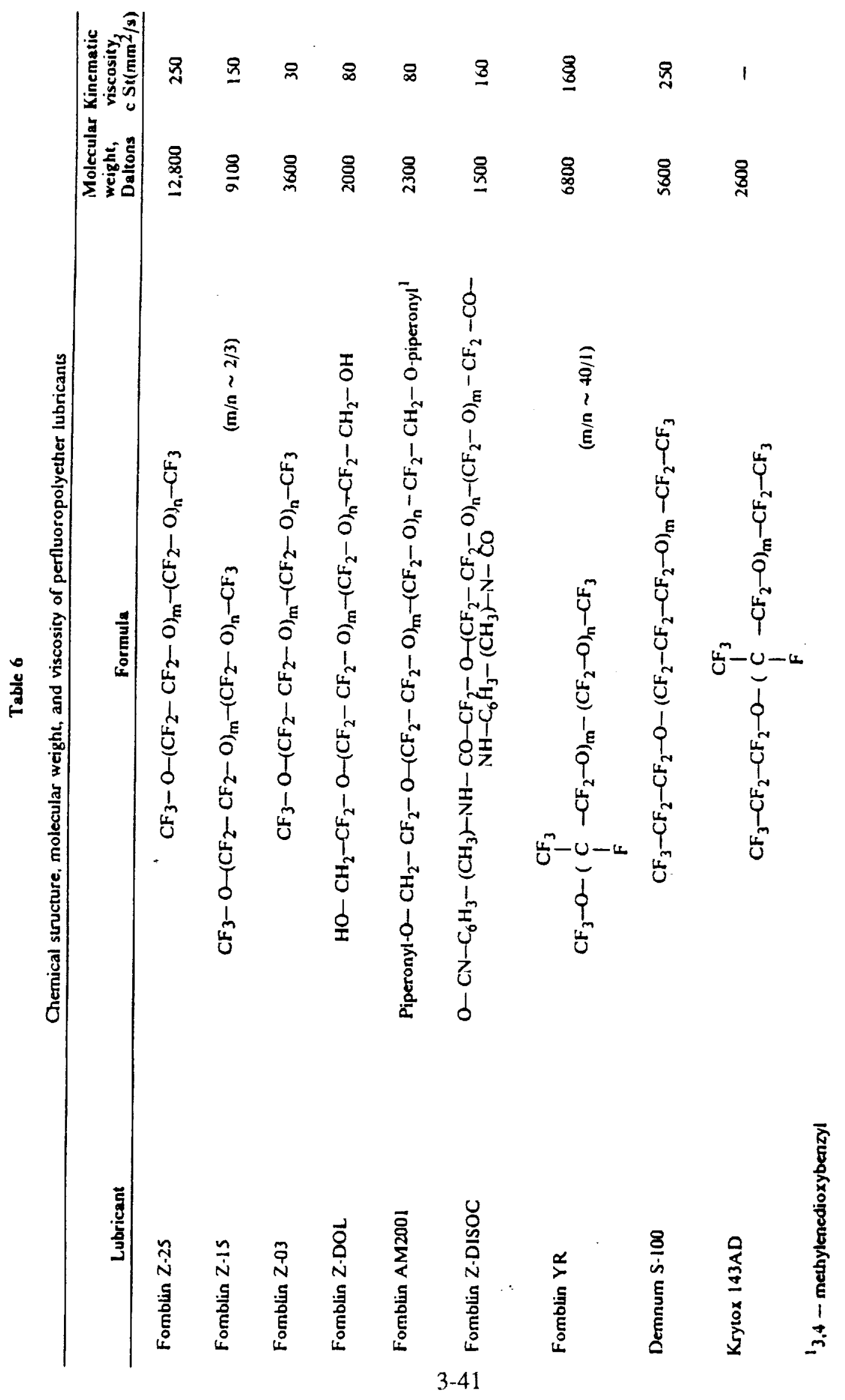




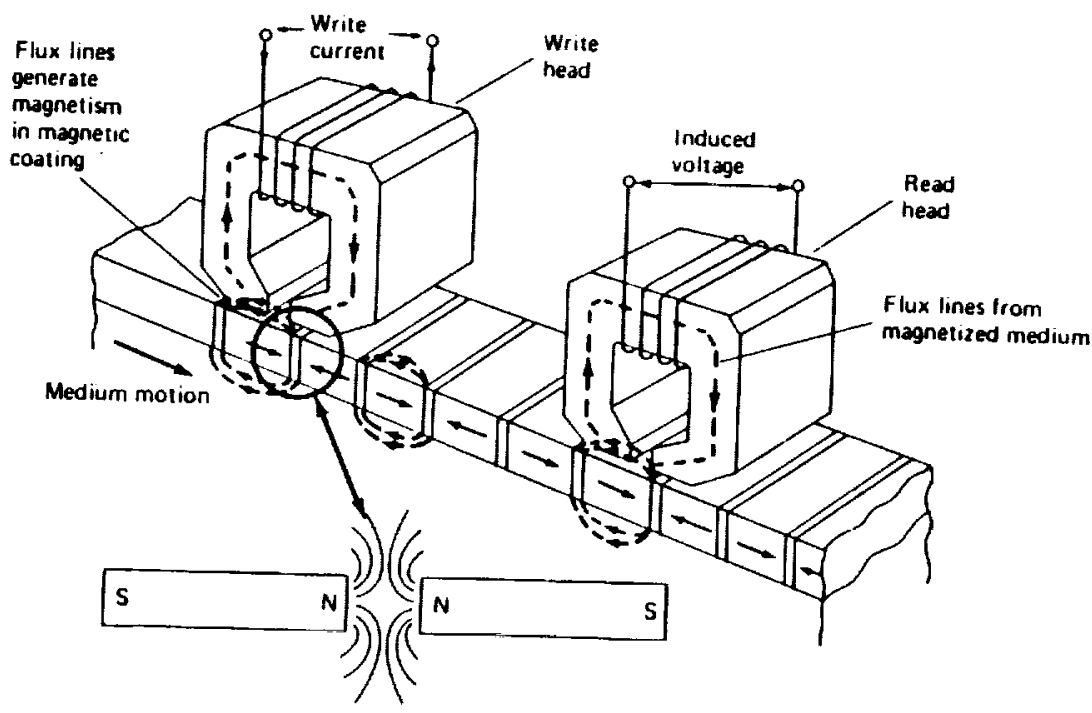

Figure 1. Principle of horizontal magnetic recording and playhack (Bhushan, 1990).

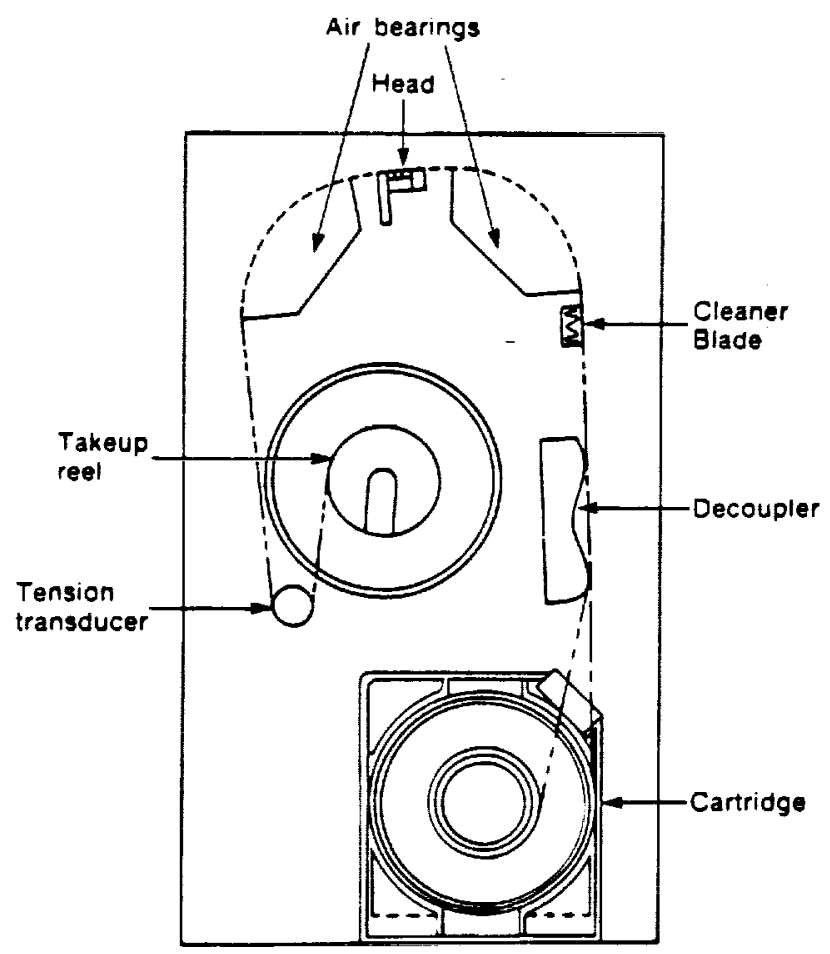

Figure 2. Schematic of tape path in an IBM 3480/3490 data-processing tape drive (Bhushan, 1990). 


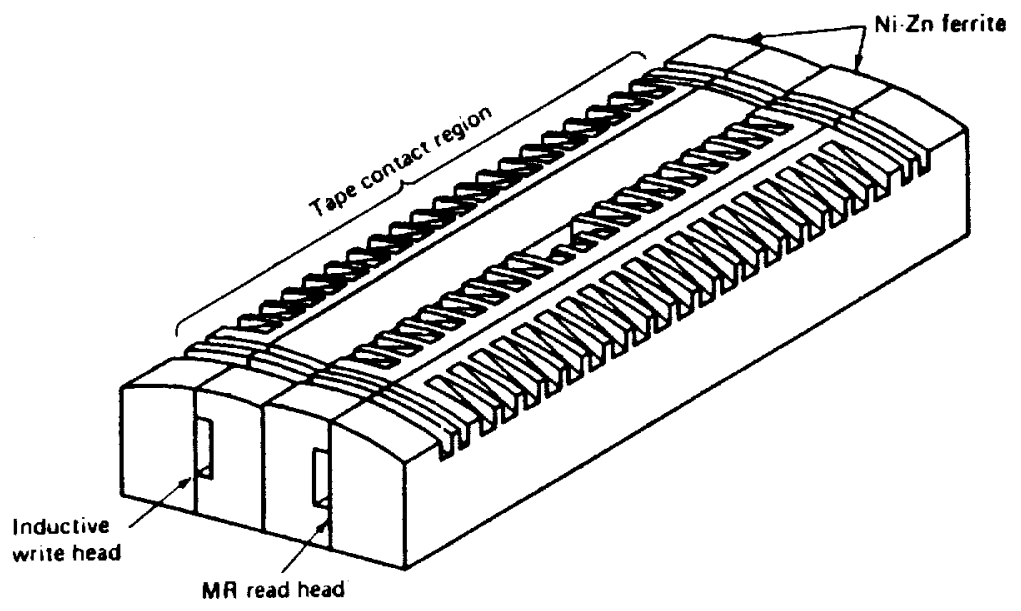

Figure 3. Schematic of a magnetic thin-film head (with a radius of cylindrical contour of about $20 \mathrm{~mm}$ ) for an IBM $3480 / 3490$ tape drive (Bhushan, 1990).

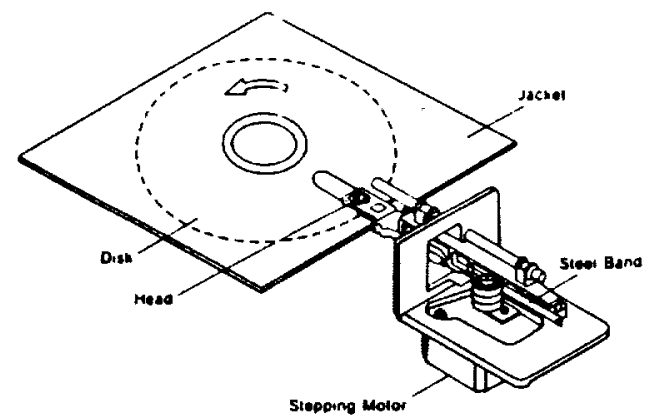

Figure 4. Schematic of the head-disk interface for fexible disk drives (Bhushan, 1990). 


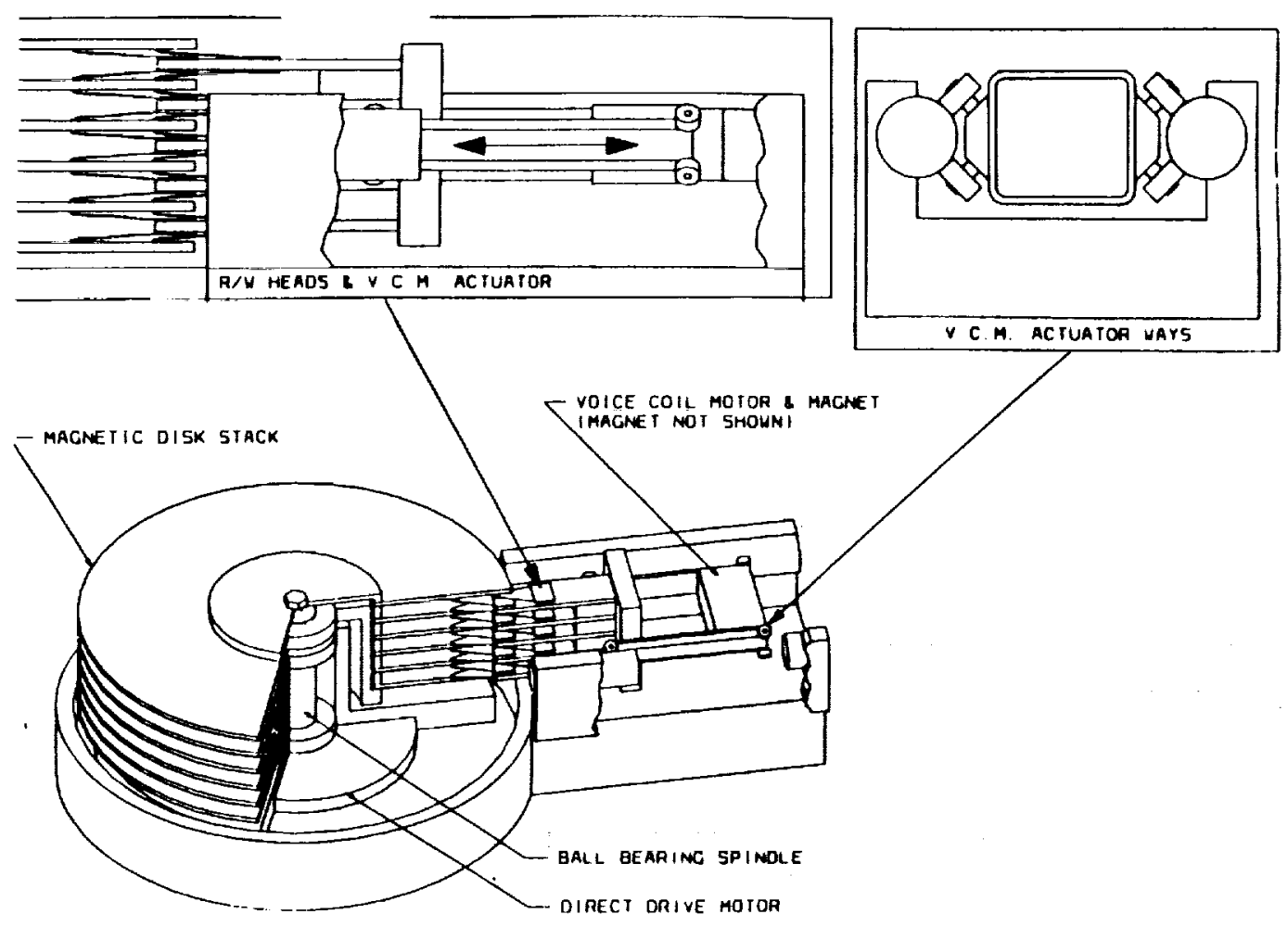

Figure 5. Schematic of the head-disk assembly in an IBM 3390-type rigid-disk drives consisting of the voice-coil-motor driven head-arm assemby and disk stack.
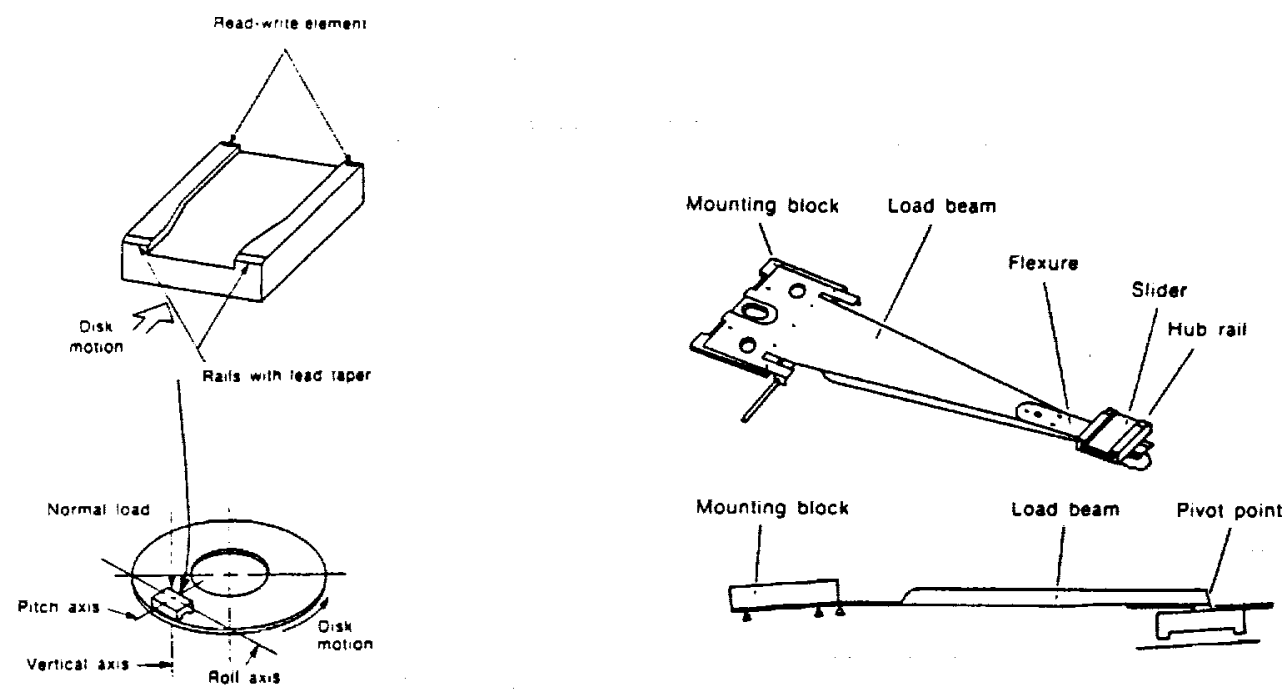

Flgure 6. Schematics of (a) the self-acting IBM 338nK/3390-type head slider on a magnetic disk. (b) IBM 3370/3380/3390-type suspension-slider assembly. 

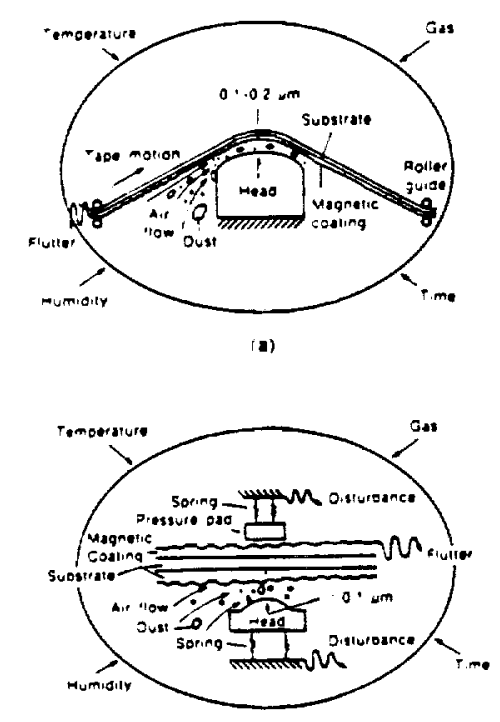

(b)

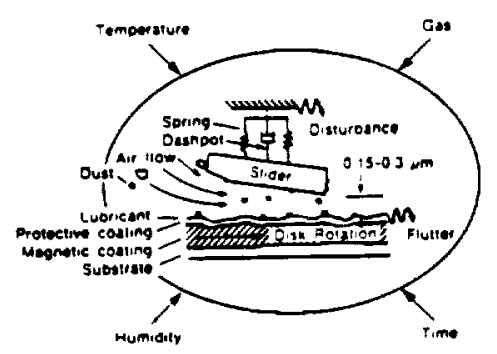

(c)

Figure 7. Schematic diagrams of various head-medium interfaces (a) head-tape interface, (b) head-flexible disk incerface, and (c) head.rigid disk interface (Bhushan,

1990).

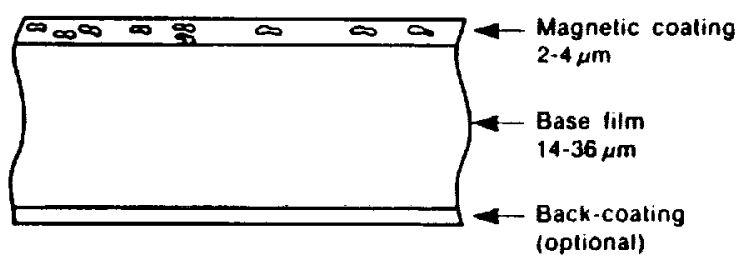

(a)

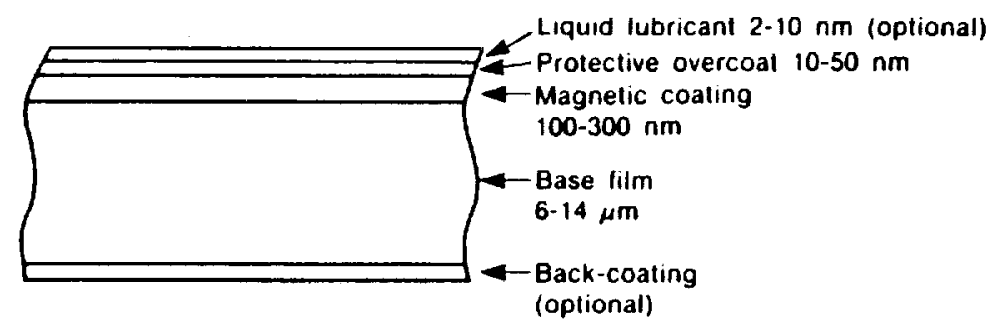

(b)

Figure 8. Sectional views of (a) a particulate, and (h) a thin-film magnetic tape. 


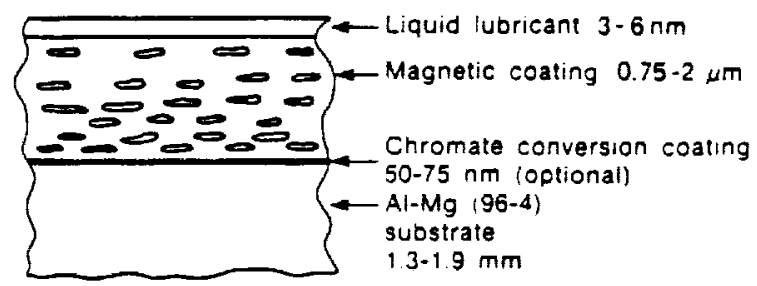

Particulate Disk

Liquid Lubricant $1-4 \mathrm{~nm}$ loptional)

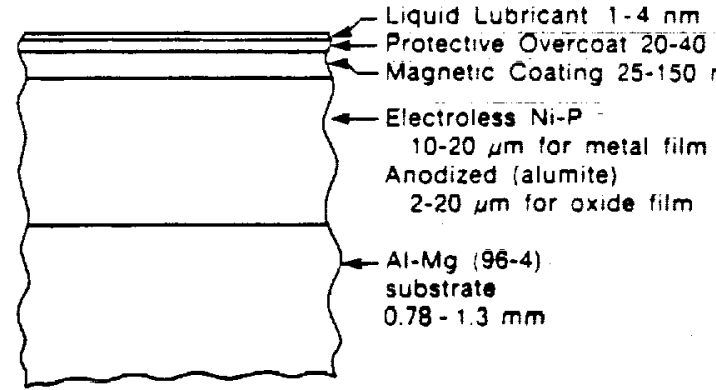

Thin-Film Disk

Figurs 9. Sectional views of (a) a particulate, and (h) a thin-film magnetic rigid disk.

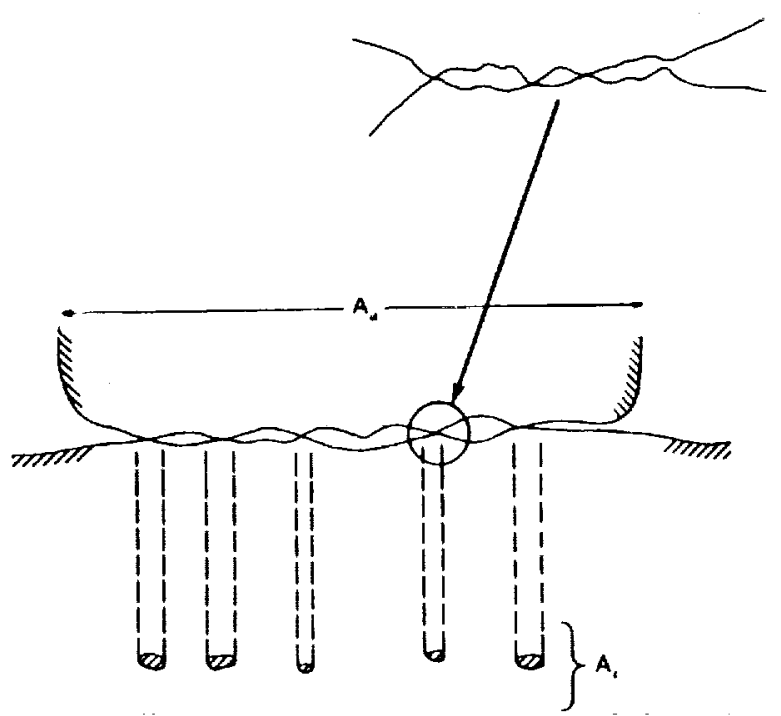

Figare 10. Schematic representation of an interface, showing the apparent and real aress of contact. Inset shows the detail of a contact on a submicron scale. Typical size of an asperity contact is from submicron to a few microns. 


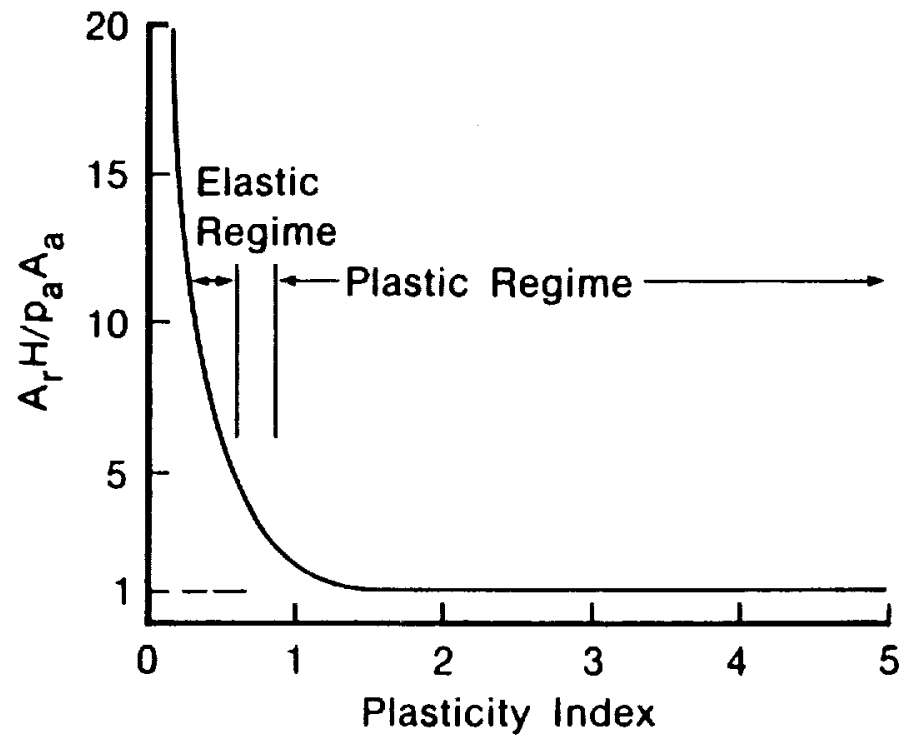

Figure 11. Influence of plasticity index on the real area of contact in metals/ceramics (Bhushan, 1990). 

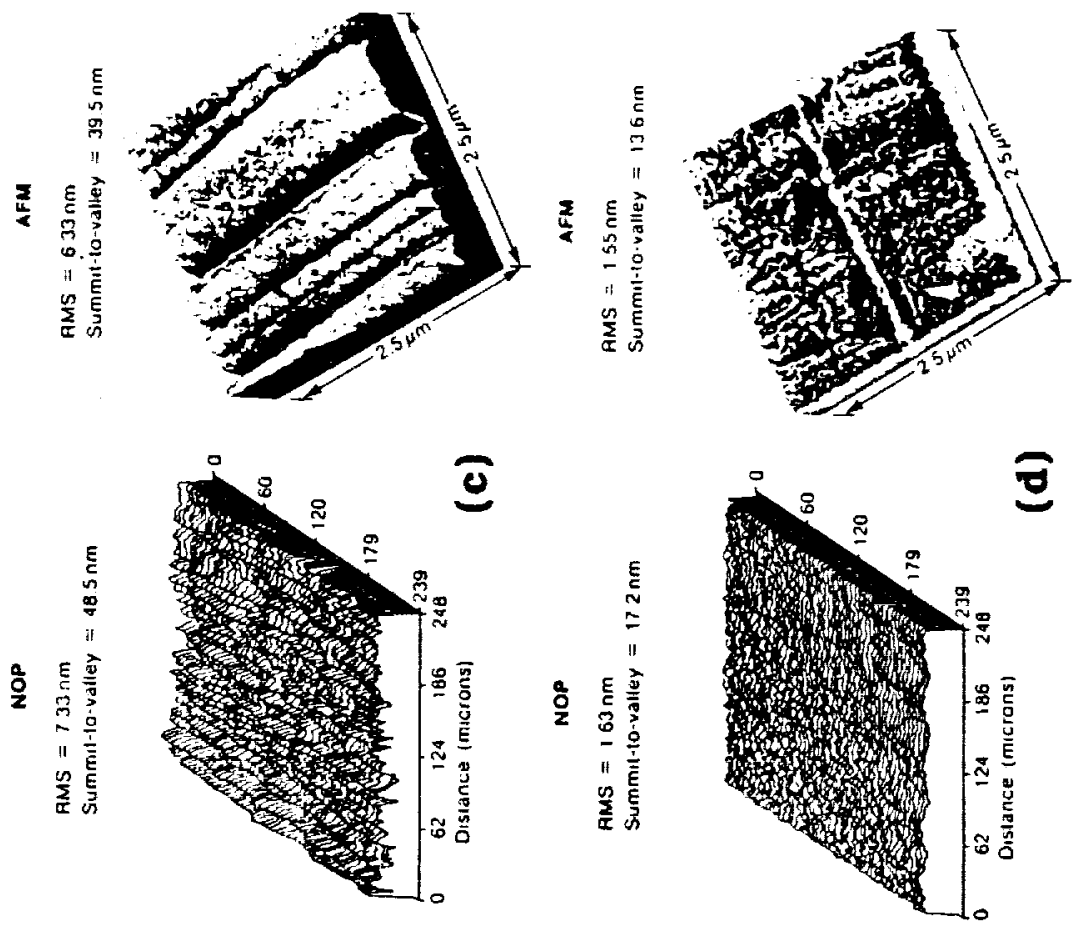


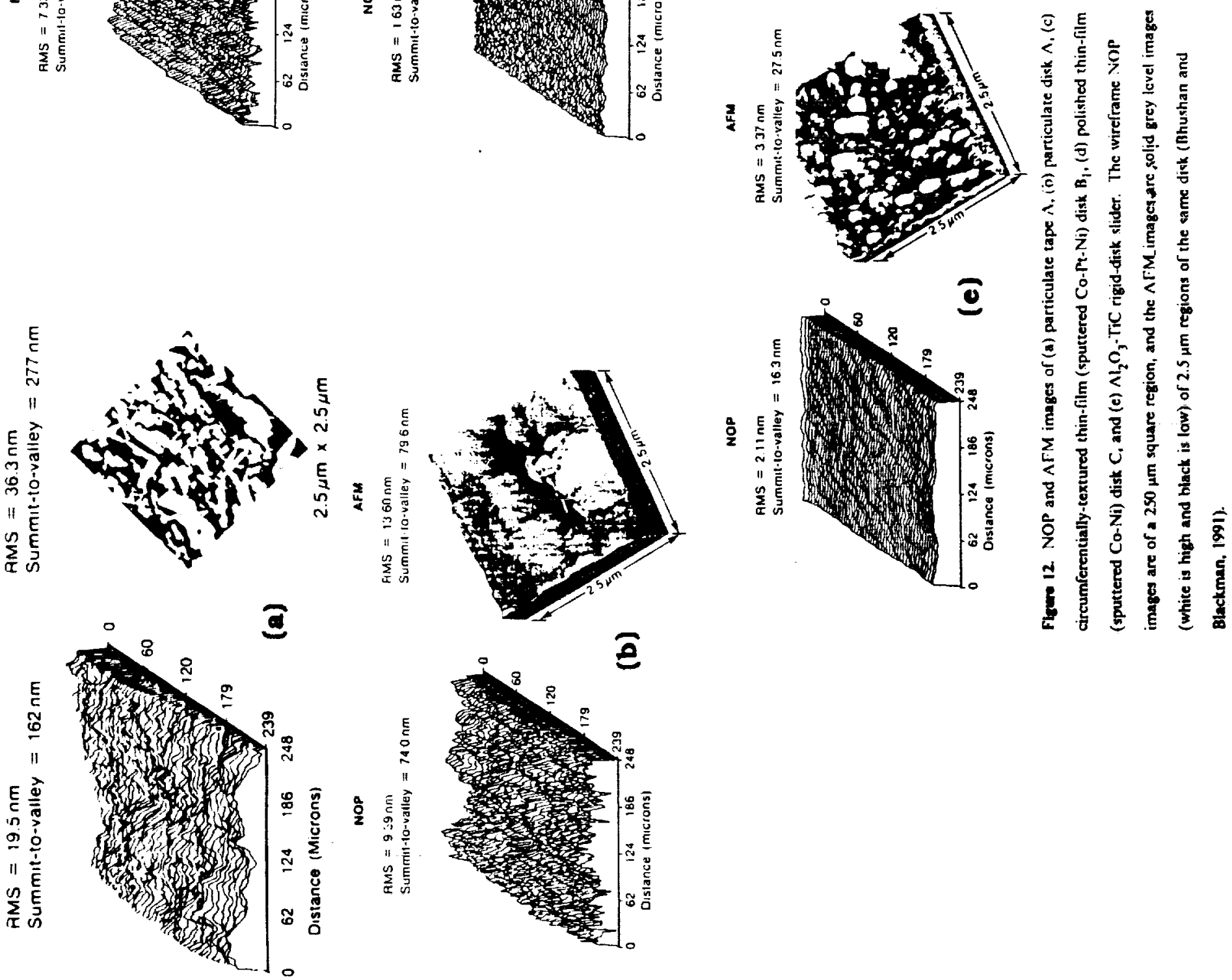


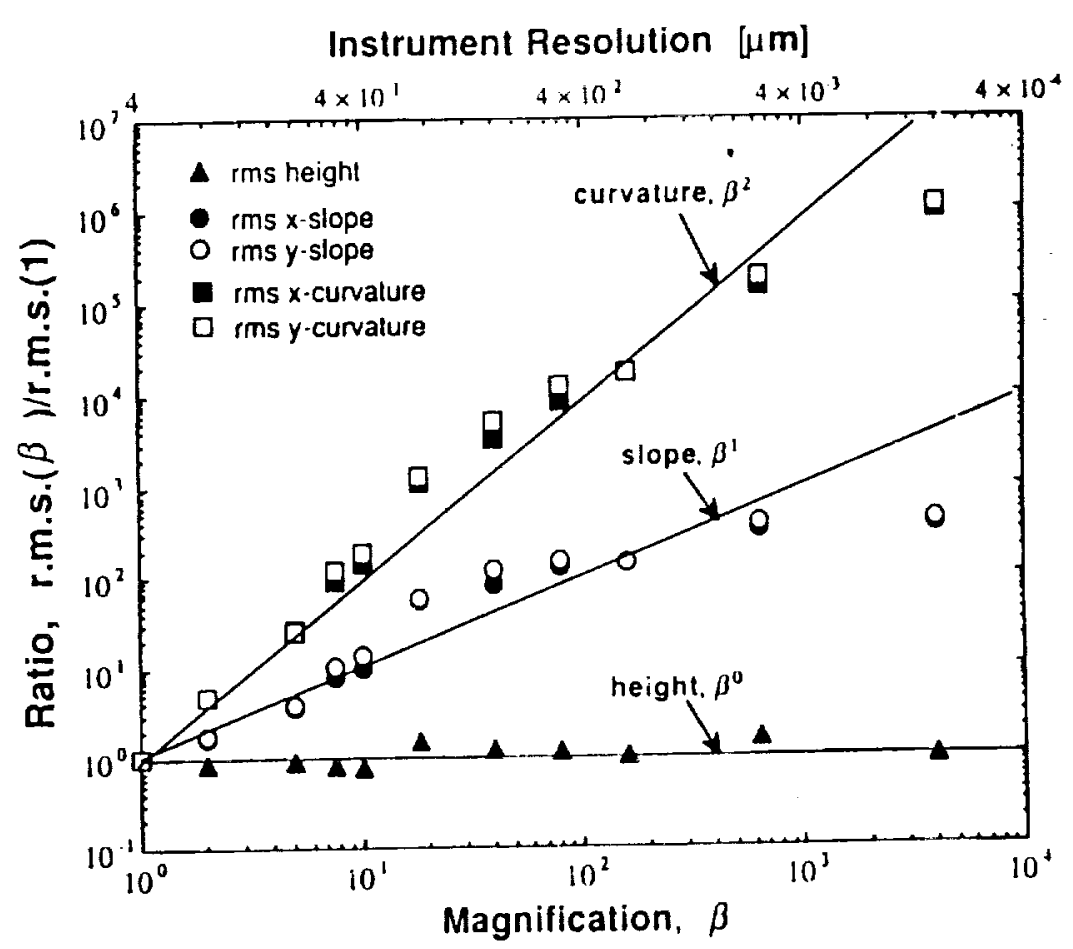

Figure 13. Variation of the ratio of the rms values at a surface magnification $\beta>0$ to the corresponding rms values at a magnification of unity, with magnification $\beta$. The data for $\beta \leq 10$ was obtained by NOP measuremente and that for $\beta \geq 1$ in was oheained by AFM (Oden et al., 1992).

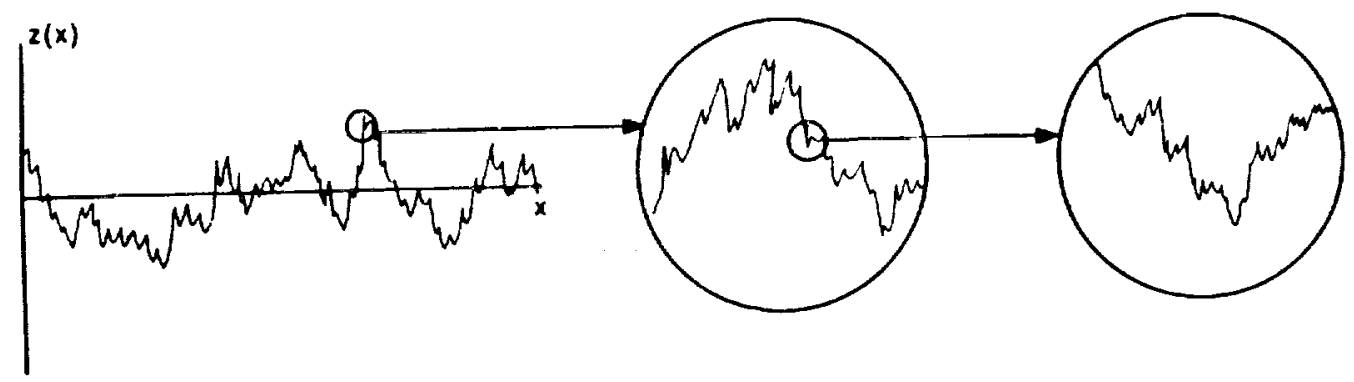

Fleure 14. Quantitative description of statistical self-afTinity for a surface profile. 

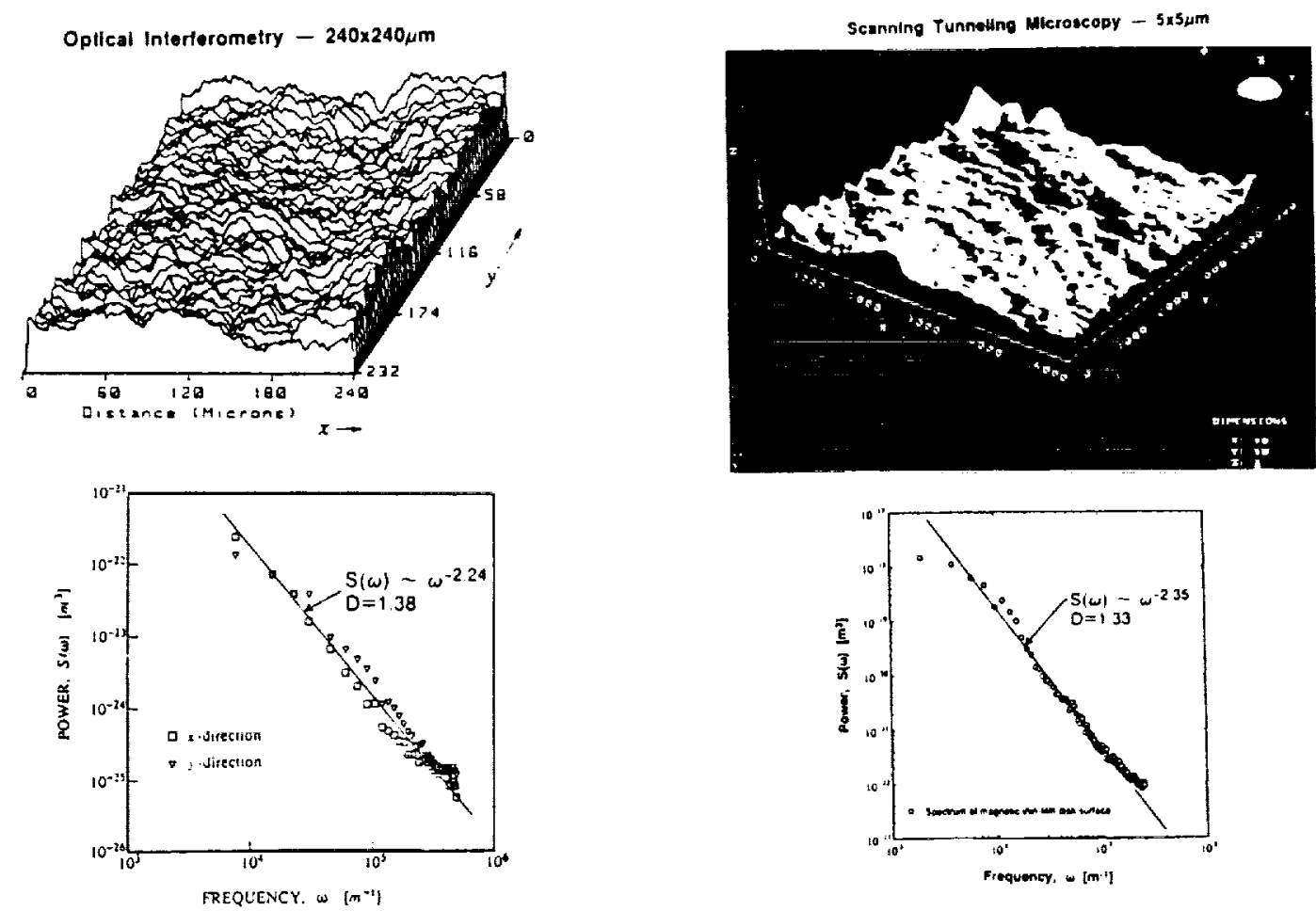

Figure 15. Surface topography and average power spectra at diferent length scales of a magnetic thin-film rigid disk ( surface (a) VOP data, (h) STM Jata (Majumdar and Bhushan, 1990).

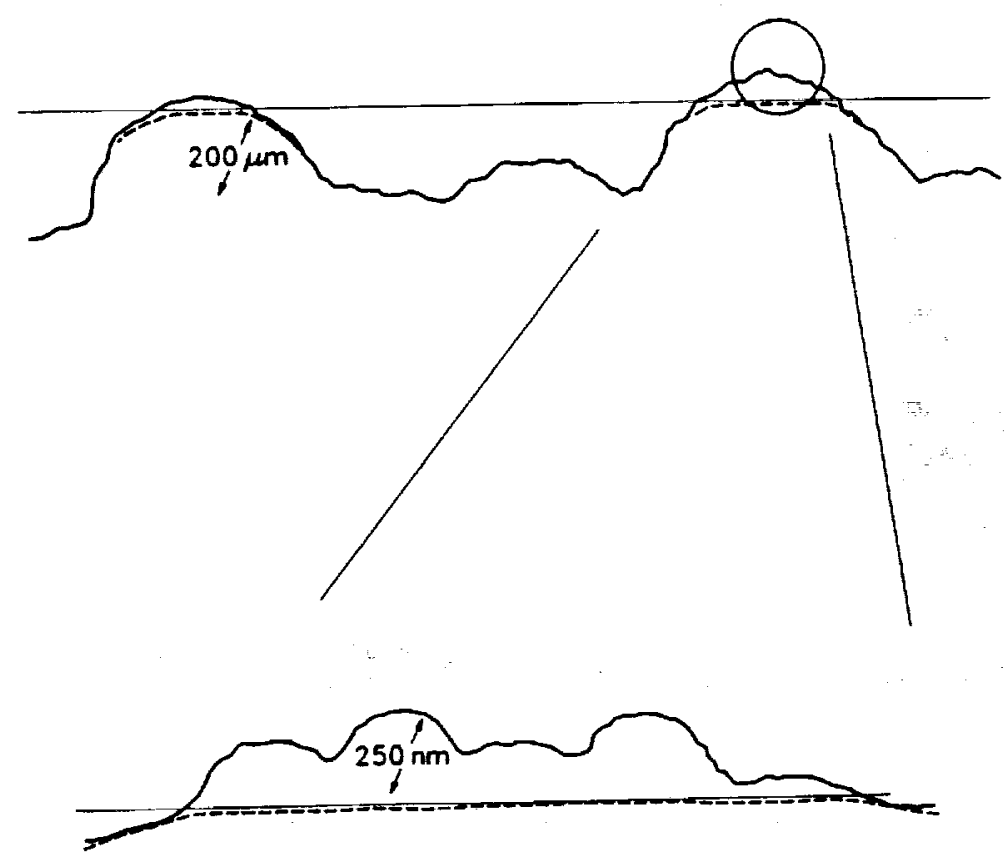

Figure 16. Schematic of local asperity deformation during contact of a mugh surface (upper profile measured by NOP and lower profile measured by $A \Gamma \mathrm{W}$, typical dimensions shown for a polished thin-film disk C) against a flat surface. The vertical axis is magniffed for clarity. Firm lines show the surfaces before contact and dotted lines show surfaces after contact (Bhushan and Blackman, 1991). 

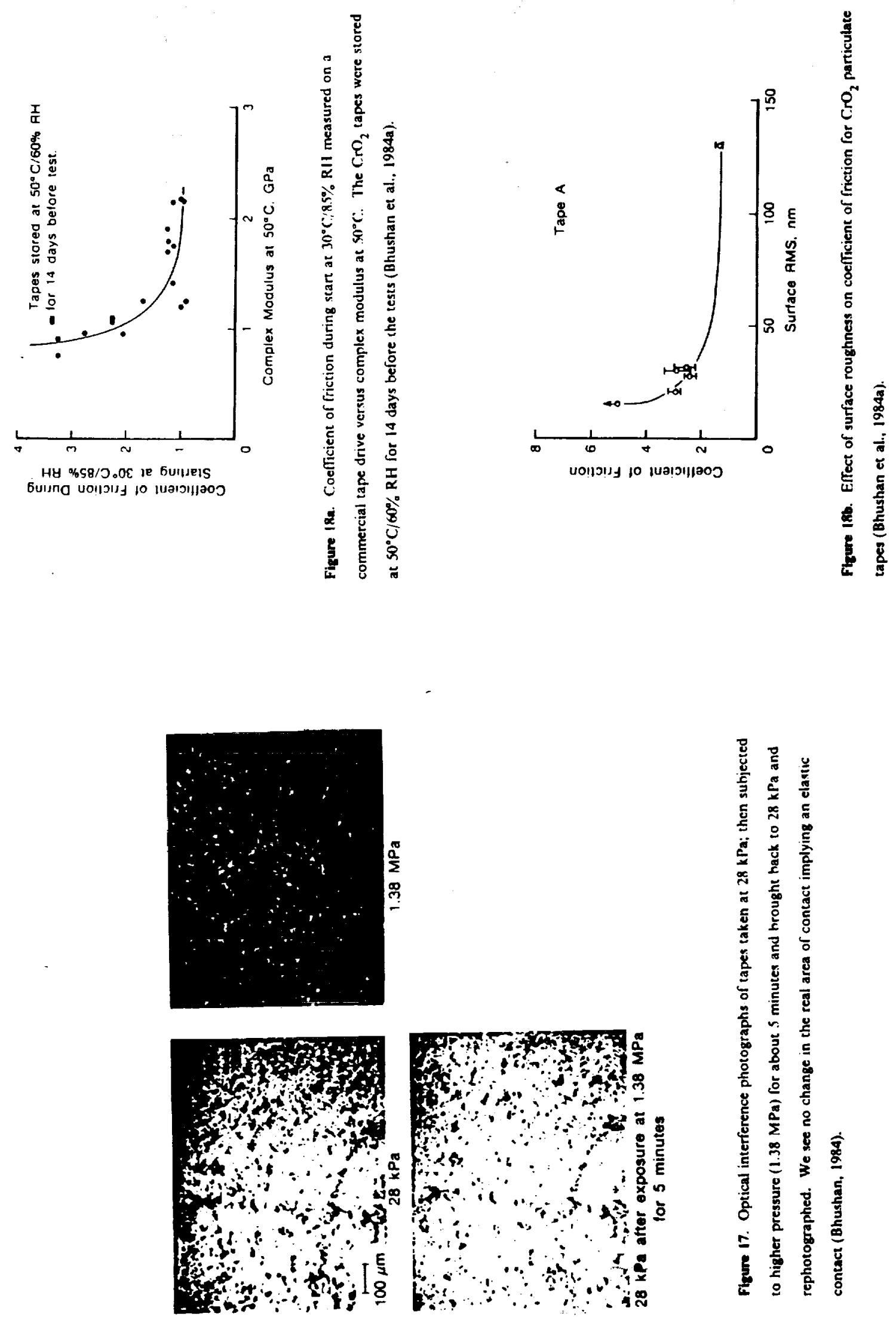


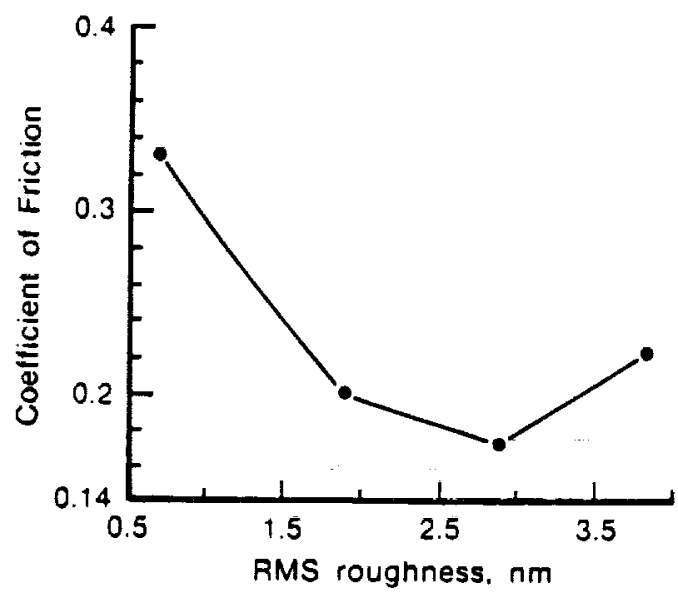

Figure 19. Coefficient of friction as a function of degrce of disk texturing for a thin-film (metal) rigid disk with sputtered carbon overcoat against ferrite slider (Doan and Mackintosh, 1988).

Aspertity contoct diameter: meon=2.36 $\mathrm{mm}$ standard der. $=2.94 \mathrm{~mm}$ Number of contacta $=4032 / \mathrm{mm}^{2}$

Froction of real area of contocl $=1.64 \pi$

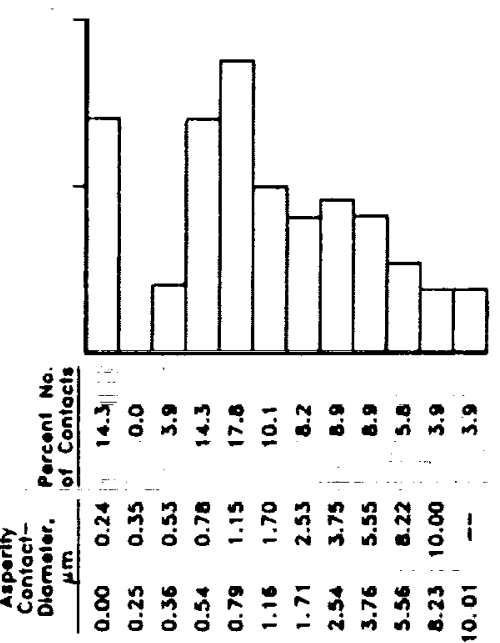

(a)
Aspertly contact diameter: mean $=3.87 \mathrm{~mm}$ standord day. $=21.58 \mathrm{~mm}$ Number of contocta $=4660 / \mathrm{mm}^{2}$

Fraction of real orea of contact $=2.51 x$

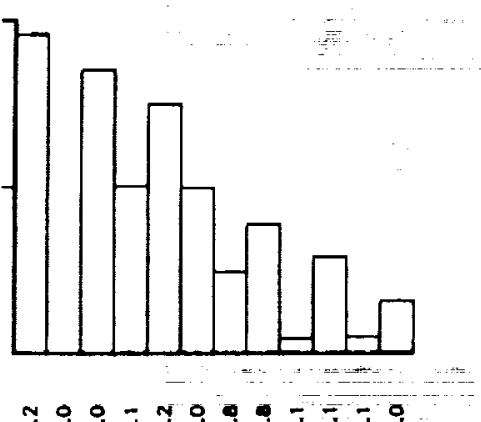

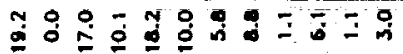

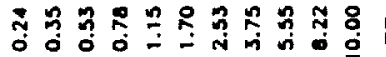

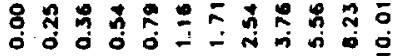

(b)

Figure 20. 1.08 normal distribution of asperity contact diameters for a thin-film disk C: loaded by $500 \mathrm{mN}(9.27 \mathrm{MPa})$ at two loading durations (s) initial and (h) after loaded for 60 hours (Bhushan and Dugger. 1990a). 

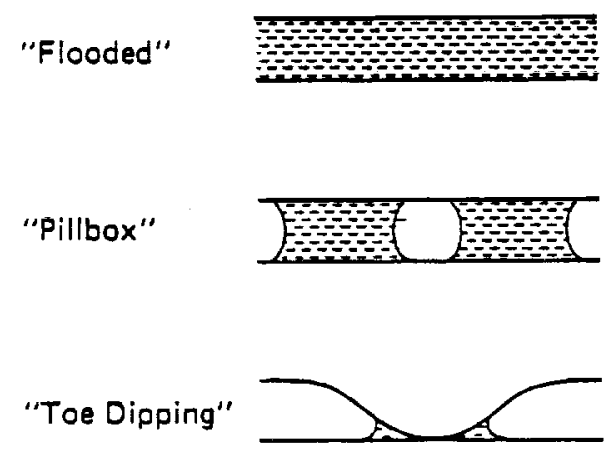

"Dry"

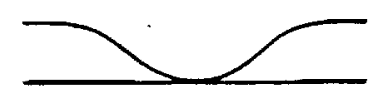

Figure 21. Regimes of difterent liquid levels in the head-medium interface.

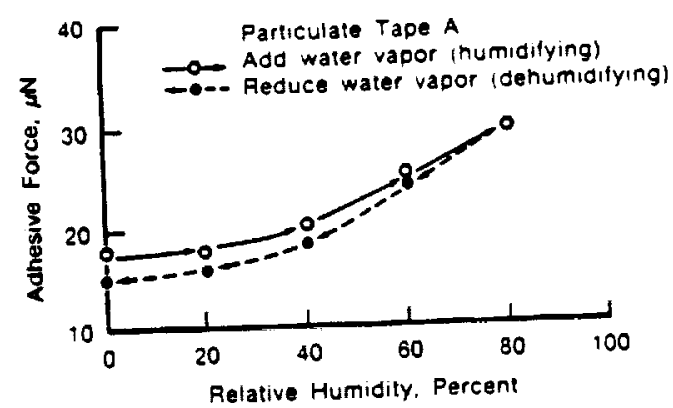

Figure 22. Effect of humidity on adhesion of $\mathrm{CrO}_{2}$ tape $A$ in contact with a $\mathrm{Vi} \cdot \mathrm{Zn}$ rerrite pin (Miyoshi et al., 1988). 

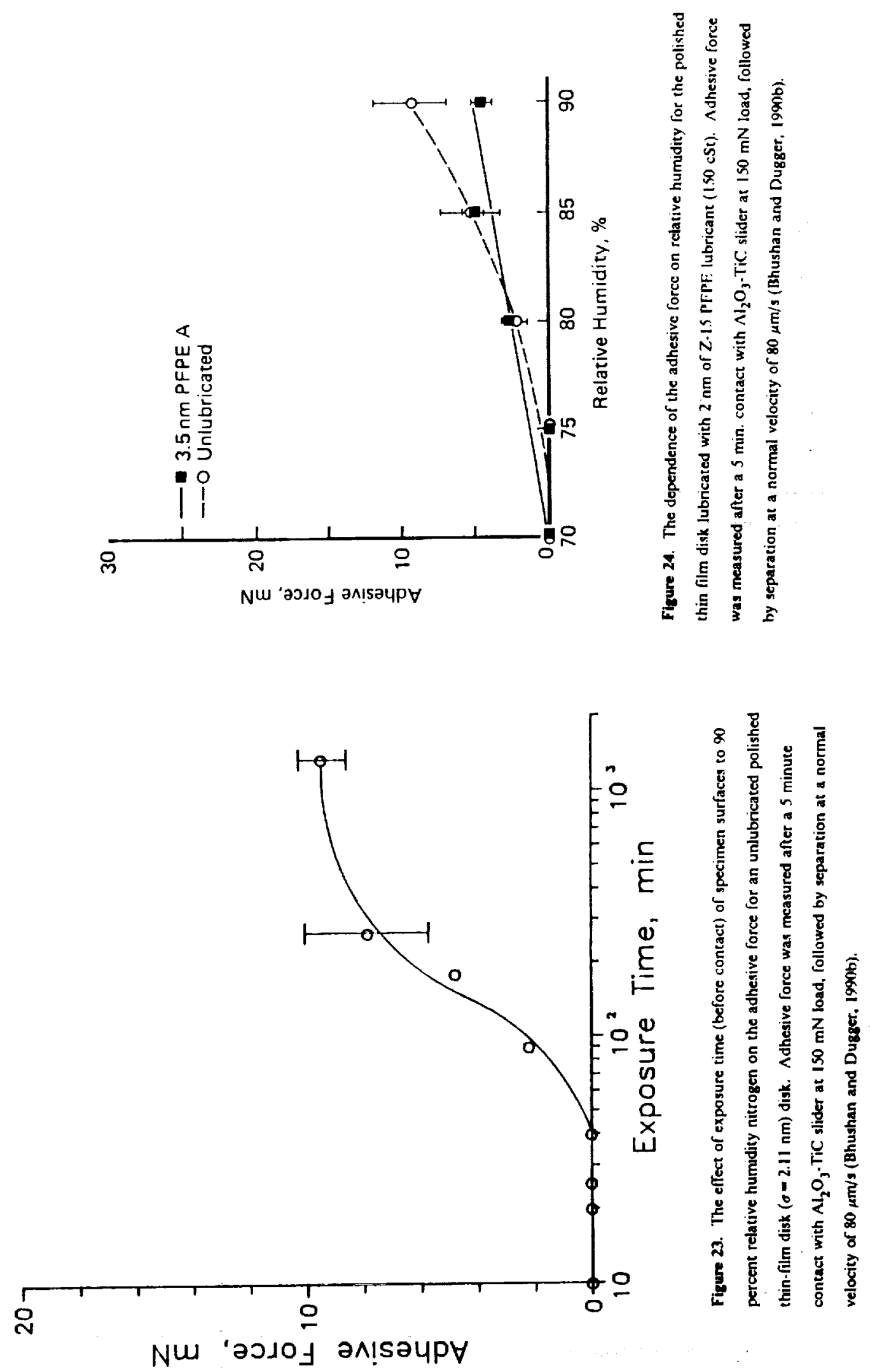

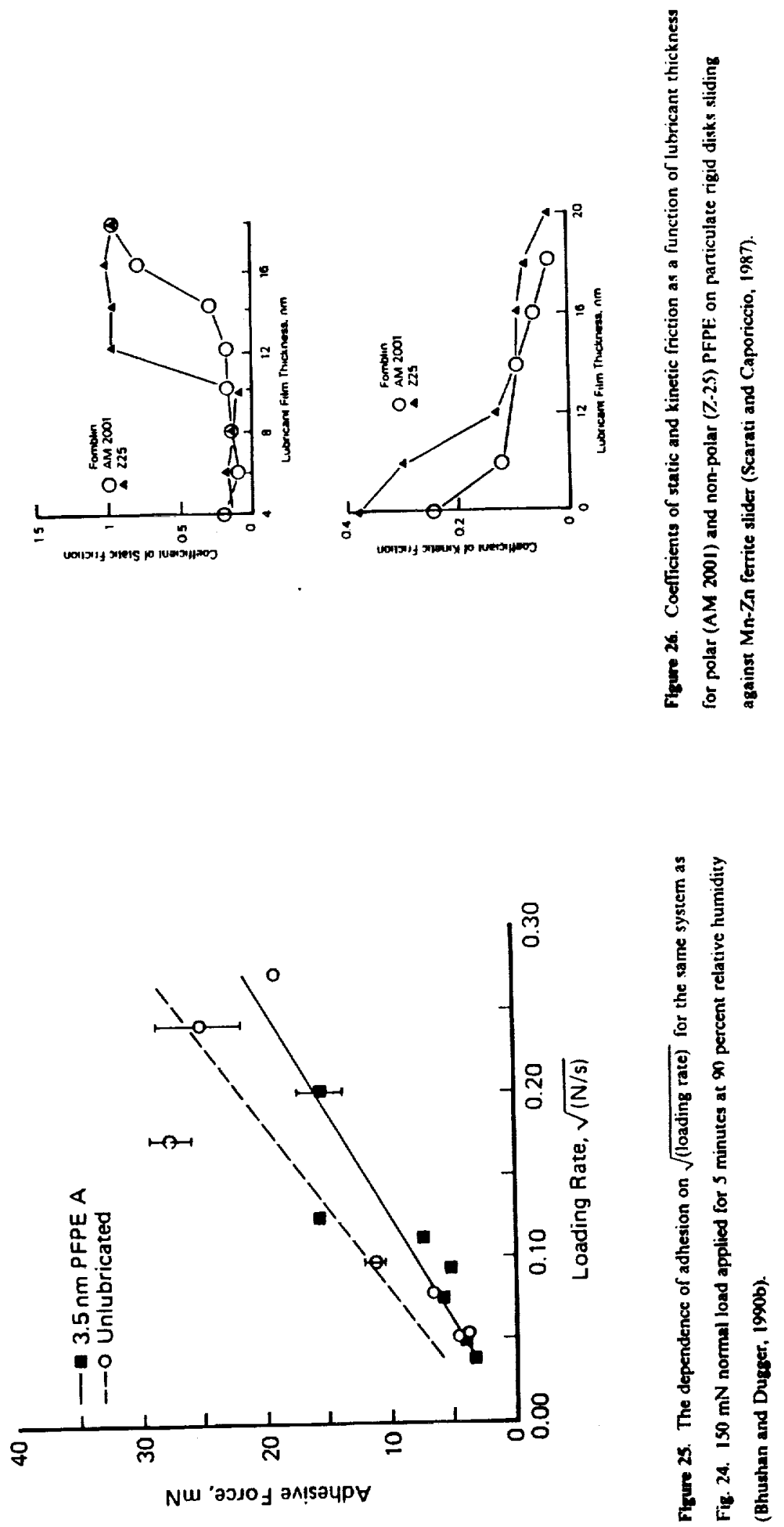

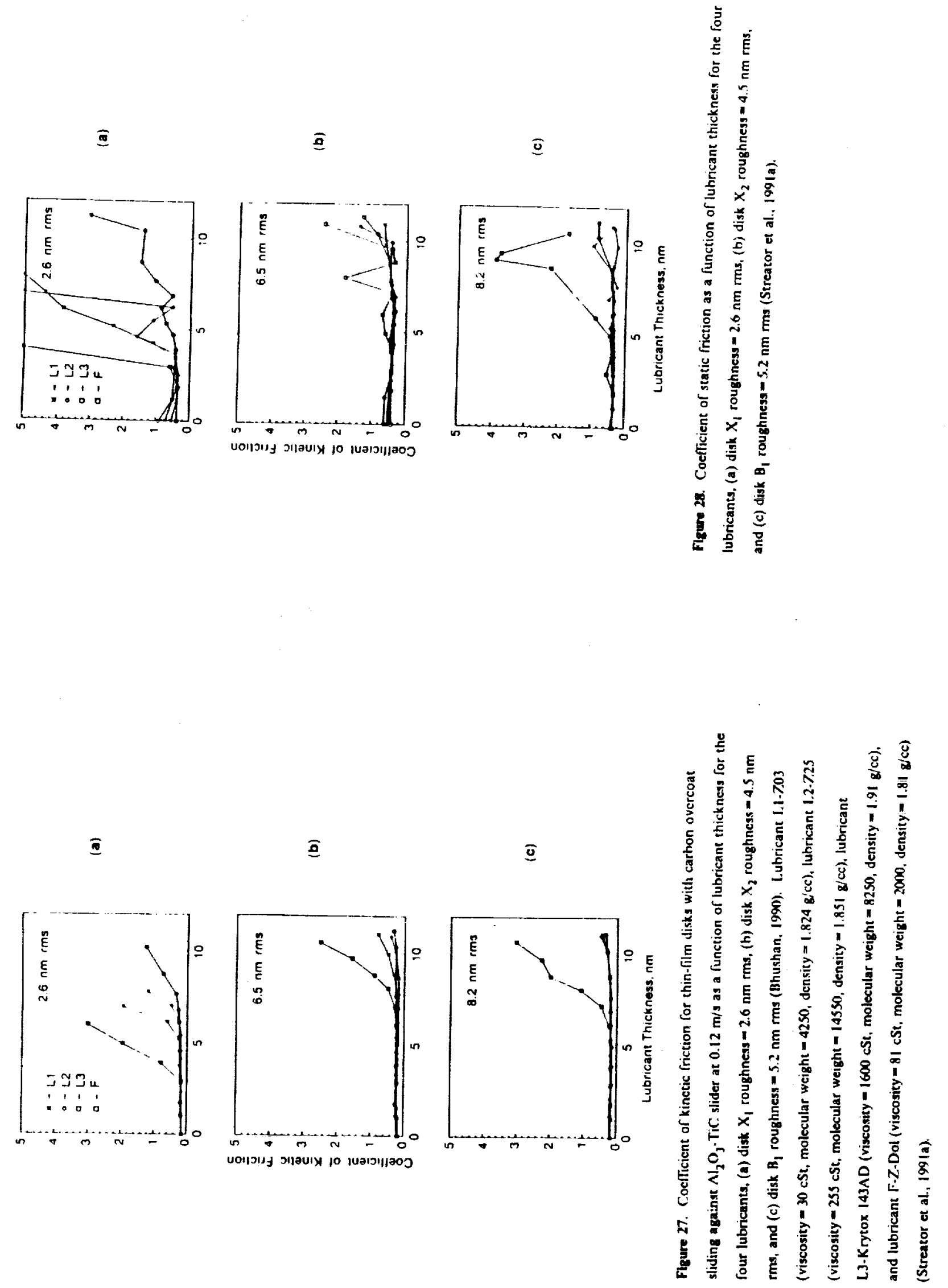


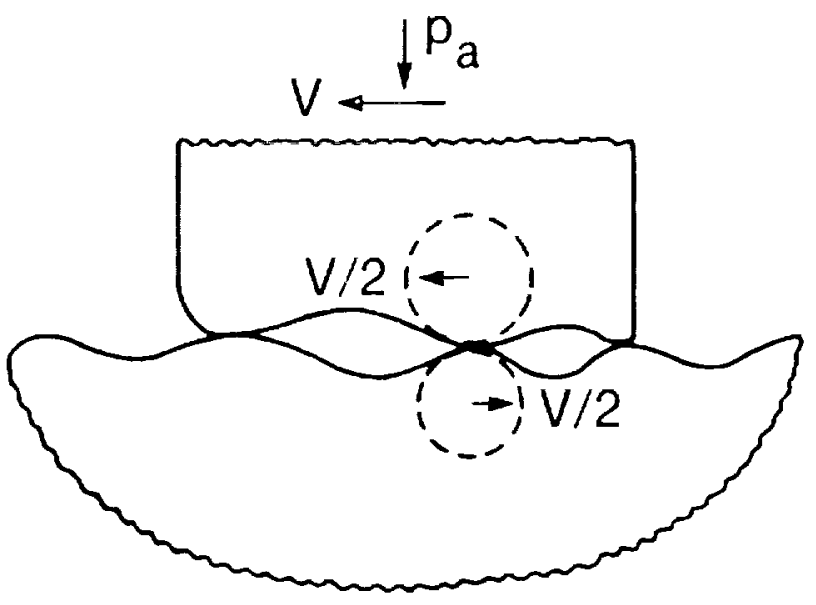

Flgure 29a. Schematic of two surfaces in contact Juring sliding at a relative sliding speed $V$ and a mean normal stress P. (Bhushan, 1987a)

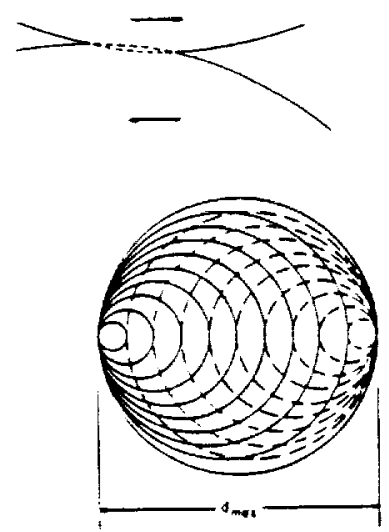

Figure 29b. The circular asperity contact growe from zero to $d_{\max }$ and then shrinks to 7era. Dotted circles show the shrinking process (Bhushan, 1987a).

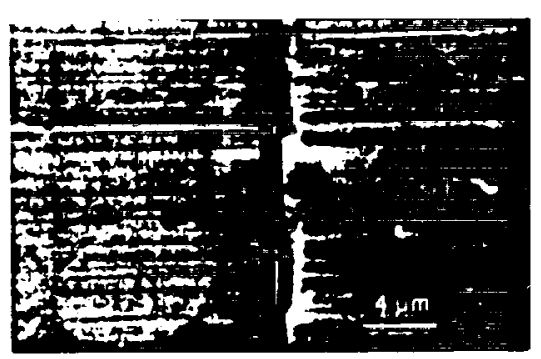

(a)

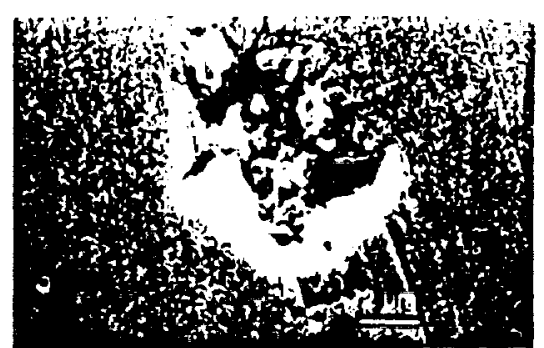

(b)

Figure 30. SEM micrographs of worn $\mathrm{Ni}-\mathrm{Zn}$ ferrite head with a $\mathrm{CrO}_{2}$ tape $\Lambda$ (a) abrasion marks (direction of tape motion-left to right), (b) surface pull out (Bhushan, 1985b). 


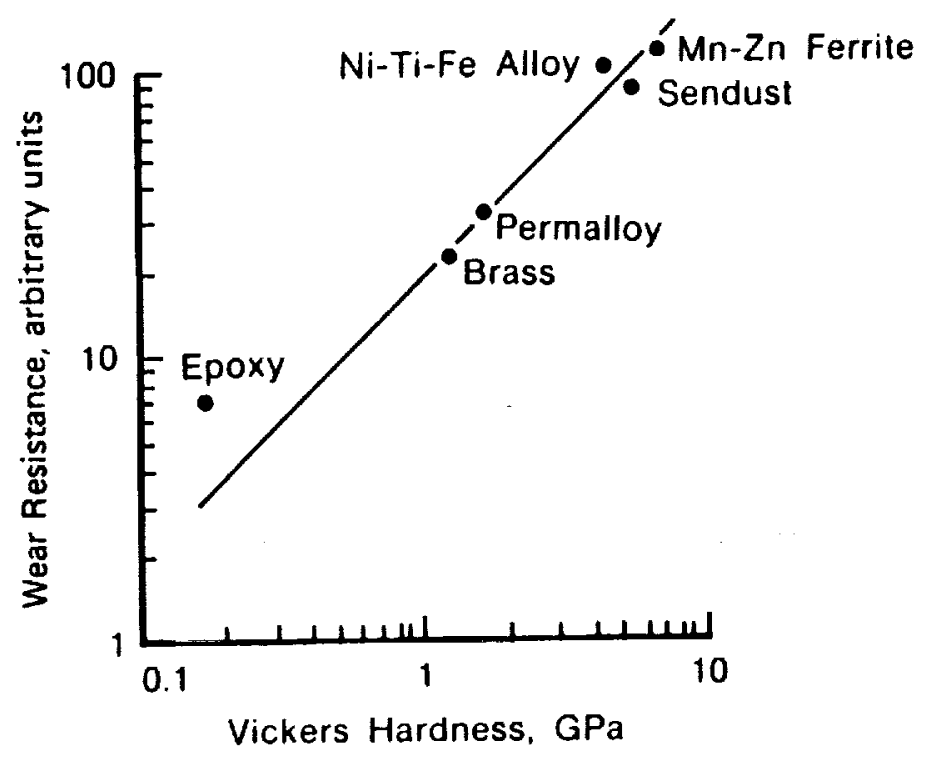

Figure 31. Wear rate of magnetic materials slid against a diamond cone as a function or Vickers hardness (Tanaka and Miyazaki, 1981).

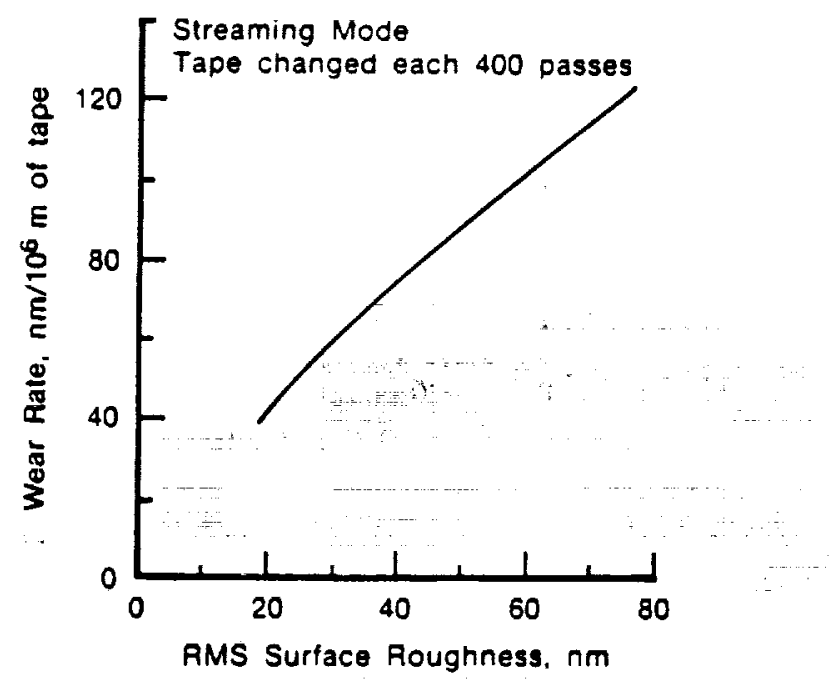

Figure 32. Ni-Zn ferrite head wear as a function of rms surface roughness of a $\mathrm{CrO}_{2}$ tape $A$ in streaming mode (Bhushan, 1985b). 


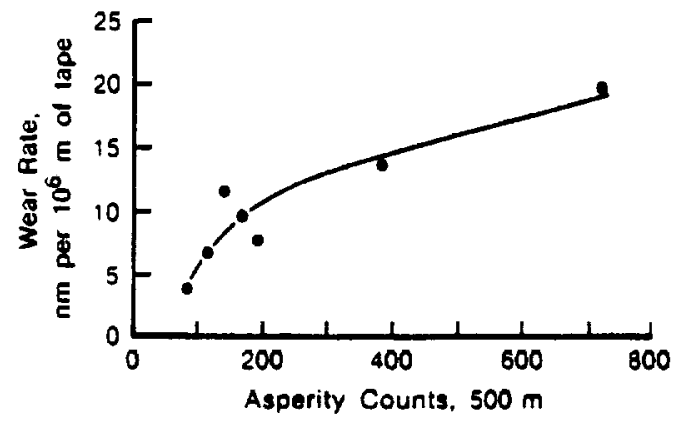

Figurs 33. Ni.7.n ferrite head wear rate as a function of asperity counts on $\mathrm{CrO}_{2}$ upe $\Lambda$ in streaming mode (Hahn, 1984).

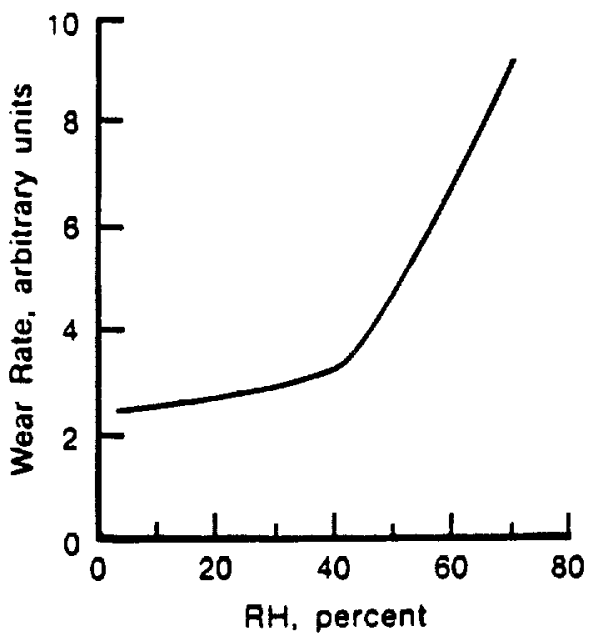

Figurs 34. Ilead wear rate with $y-\mathrm{Fe}_{2} \mathrm{O}_{3}$ tape as a Runction of relative humidity (Kelly, 1982). 


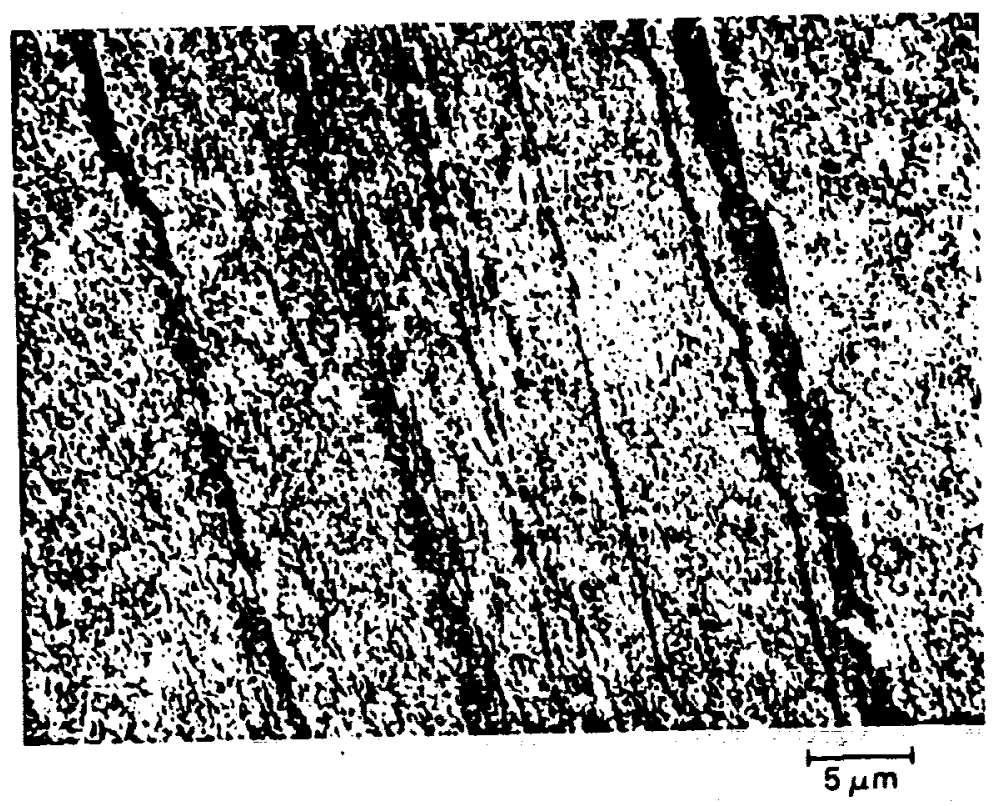

Figure 35. SFM micrograph of a worn particulate disk surface against $\mathrm{N}_{2} \mathrm{O}_{3}$ - -TiC. stider after extended use in CSS (Bhushan, 1990).

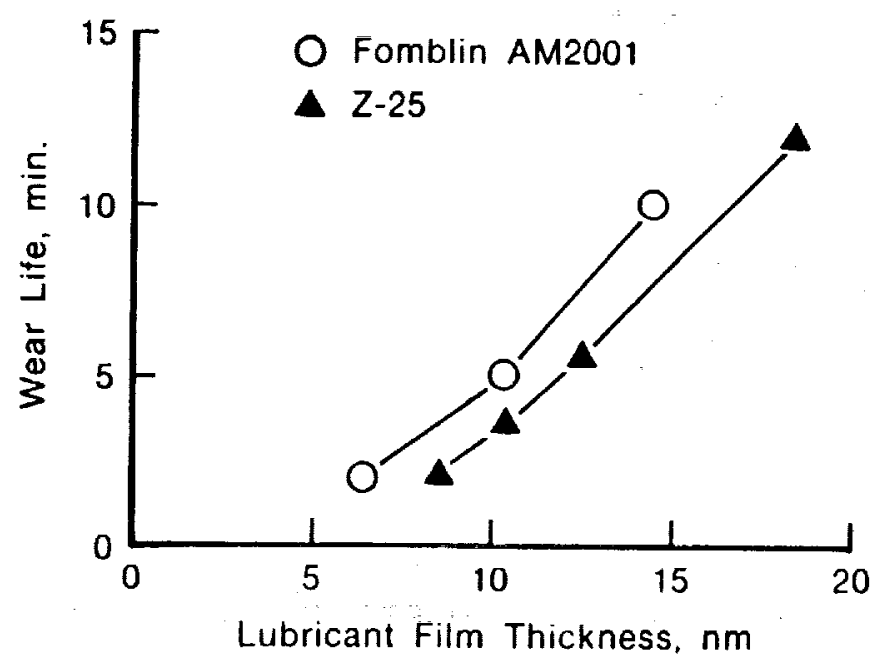

Figure 36. Wear life as a function of lubricant film thickness on a particulate rigid disk slid against Mn-7n ferrite slider (Scarati and Caporiccio, 1987). 

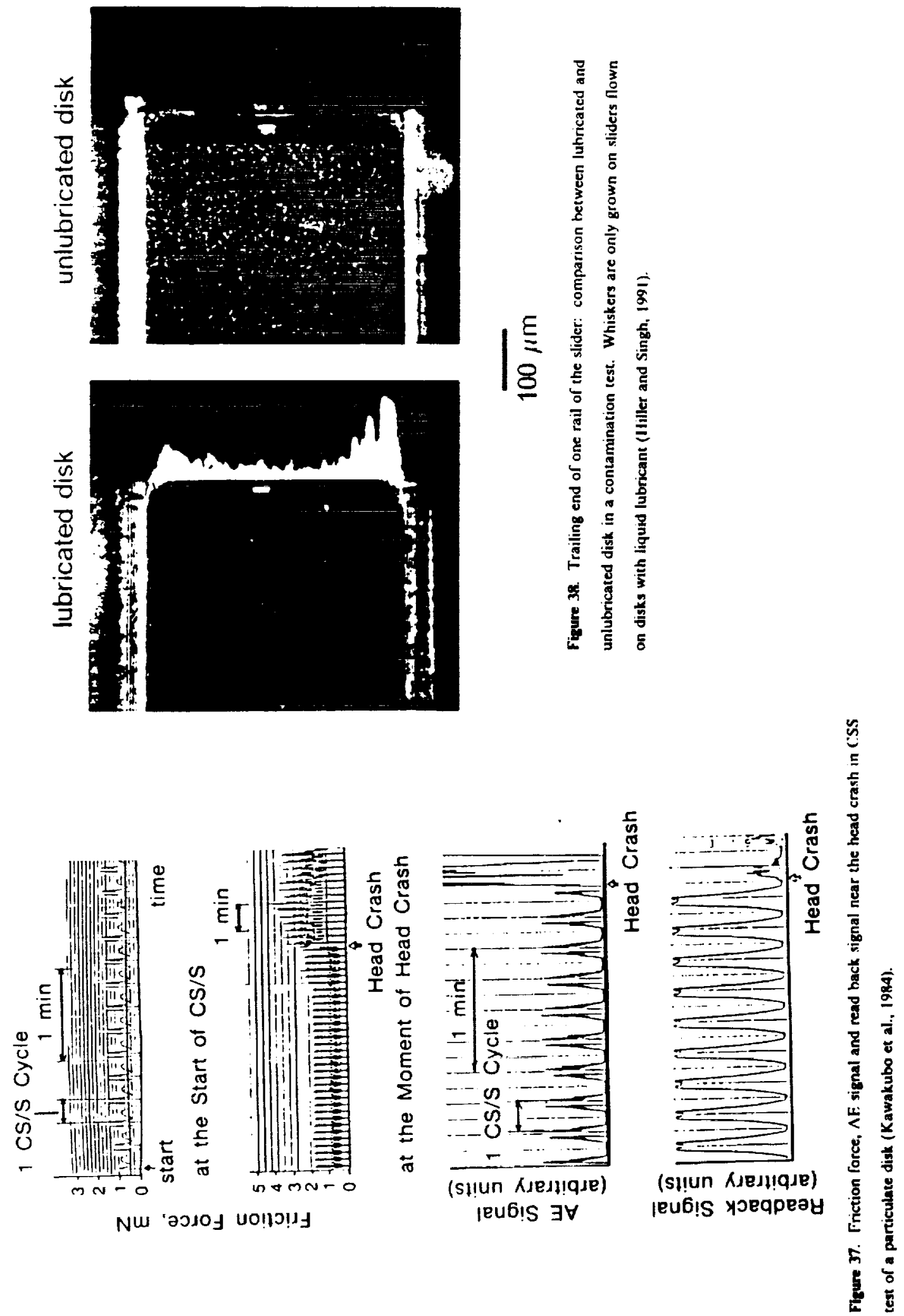


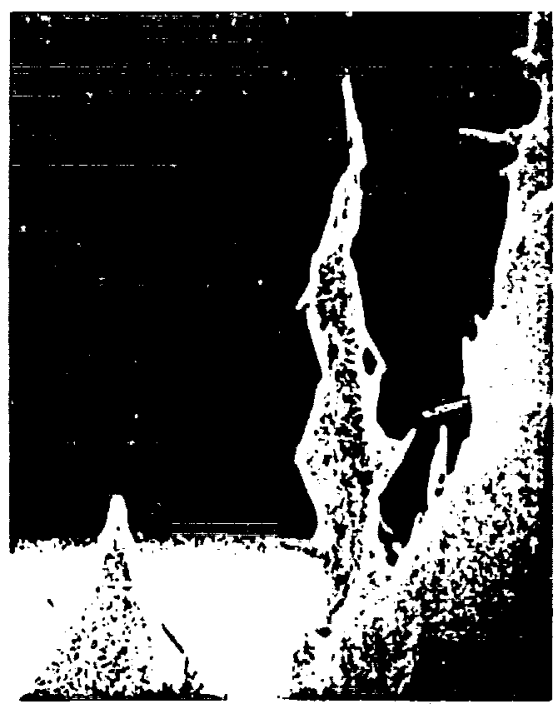

$5 / 1 \mathrm{~m}$

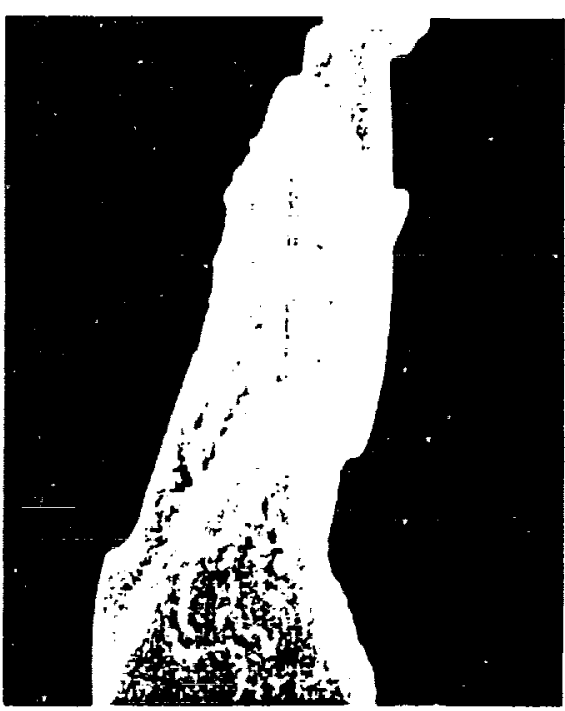

$5 \mu \mathrm{m}$

Figure 39. All polystyrene particles in the whiskers are teformed, evidence of interaction with the interface (Hiller and Singh, 1991).

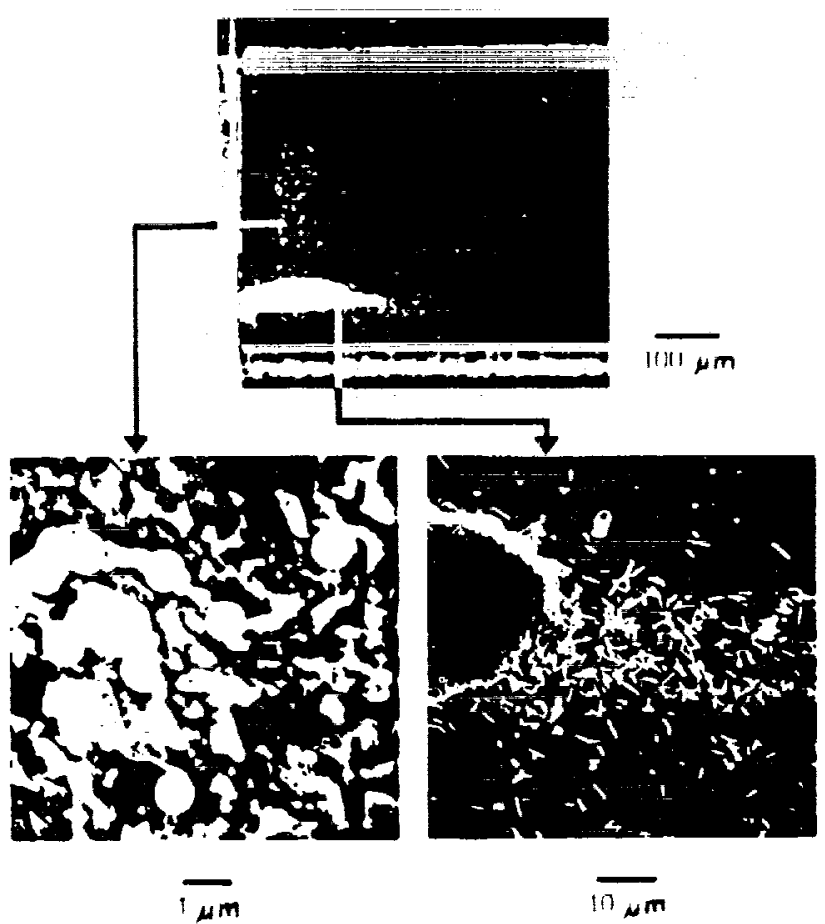

Fgore 40. Dark-field photograph of the taper showing a well-Jefined uniform particle deposition pattern and a large agglomerate (top): SF. Photographe of epherical particles within the uniform patters (lower left) and of deformed particles in the agglomerate which is a former whisker from the trailing end (lower righe) (Ililler and Singh, 1991). 


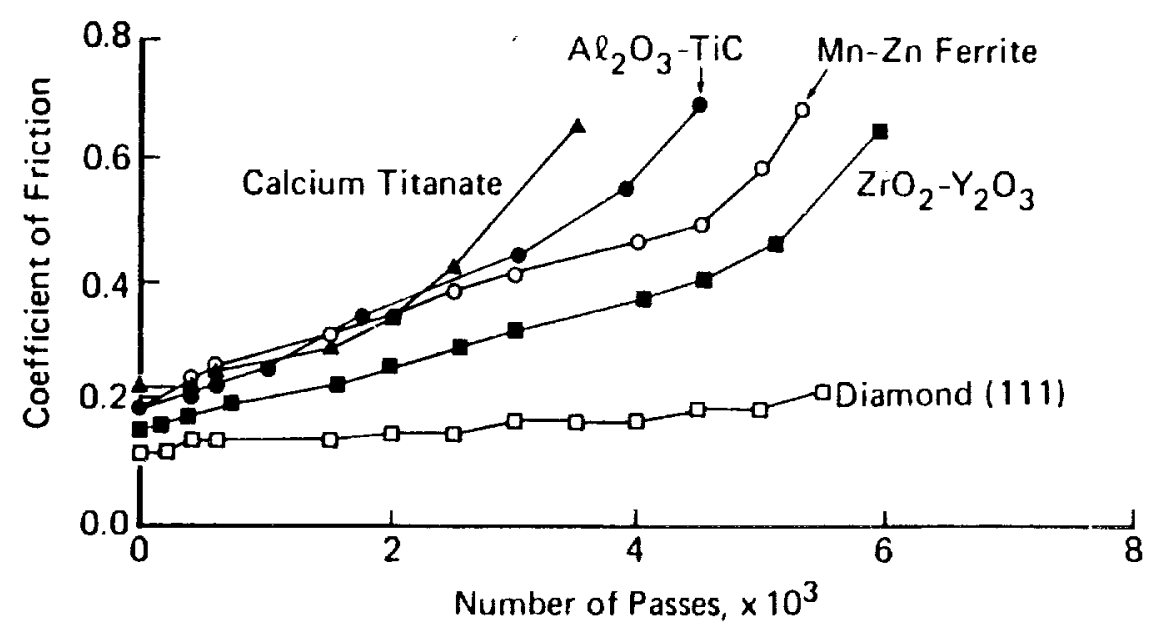

Flgurs 41. Change of the coefficient of friction of five ceramic sliders while sliding against a thin-disk disk with a carbon overcoat and perfluoropolyether as the topical lubricant (disk $B_{1}$ ) with number of stiding passes. Normal load $=1.50 \mathrm{mN}$, sliding velocity $=2.1 \mathrm{~m} / \mathrm{s}$ (Chandrasekar and Bhushan, 1991).

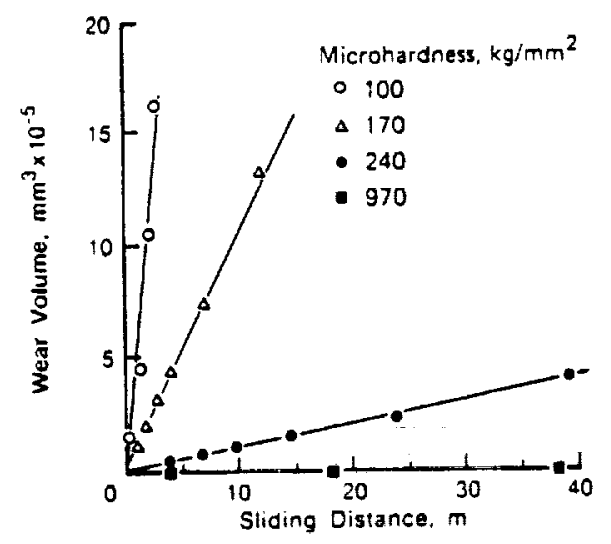

Figure 42. Relation between siding distance and wear volume for $\mathrm{SiO}_{2}$ films of different Vickers hardnesses, slid against $\mathrm{N}_{2} \mathrm{O}_{3}$. TiC slider (Yanagisawa, 198.5h). 


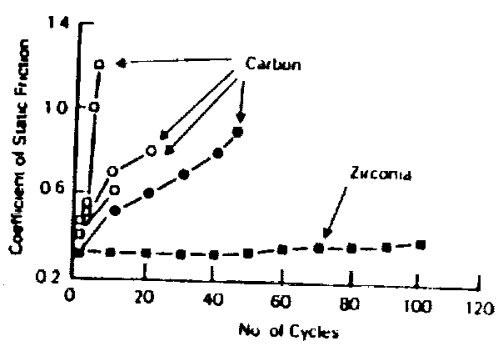

Figure 43. Coefficient of static friction as a function of number of C:SS cycles for unlubricated carbon and $7 \mathrm{rO}_{2}-\mathrm{Y}_{2} \mathrm{O}_{3}$ overcoated thin-film (metal) disks (disks $\mathrm{H}_{1}$ and $\mathrm{B}_{2}$ with no lubricant) slid against $\mathrm{Nl}_{2} \mathrm{O}_{3}$ - TiC slider (Yamashita et al., 1988).
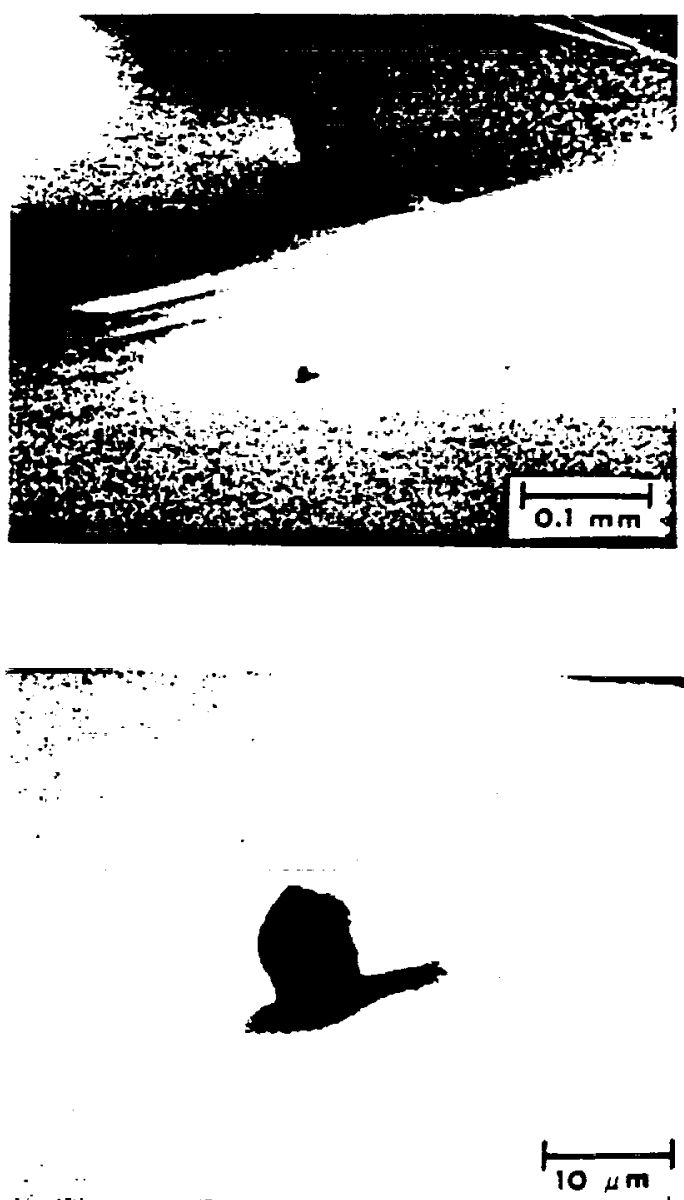

Figure 44. Appearance of the trailing edge of the $\mathrm{Al}_{2} \mathrm{O}_{3}-\mathrm{TiC}$ slider while sliding against a thin-film disk with a zirconia overcoat (disk $B_{2}$ ). Right micrograph shows a particle which is removed from the head and is sitting on the disk surfece. This photograph was taken after $7200 \mathrm{~mm}$ of sliding at $50 \mathrm{~mm} / \mathrm{min}$. (Calahrese and Bhushan, 1990). 


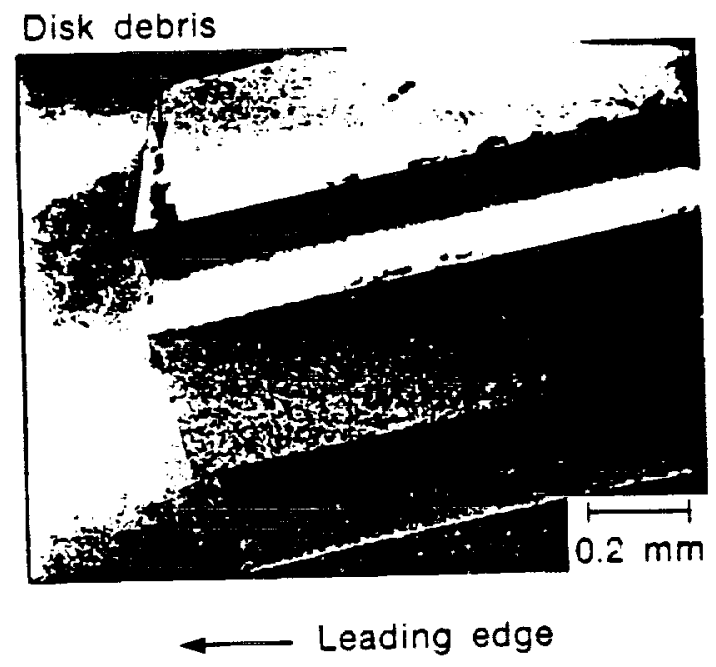

Figurs 45. Appearance of leading edge of the $\mathrm{Al}_{2} \mathrm{O}_{3}$. TiC: slider after sliding against the disk $B_{2}$. Wear debris is attached to the rail sides and the sides of the elider (Calahresc and Bhushan, 1990).

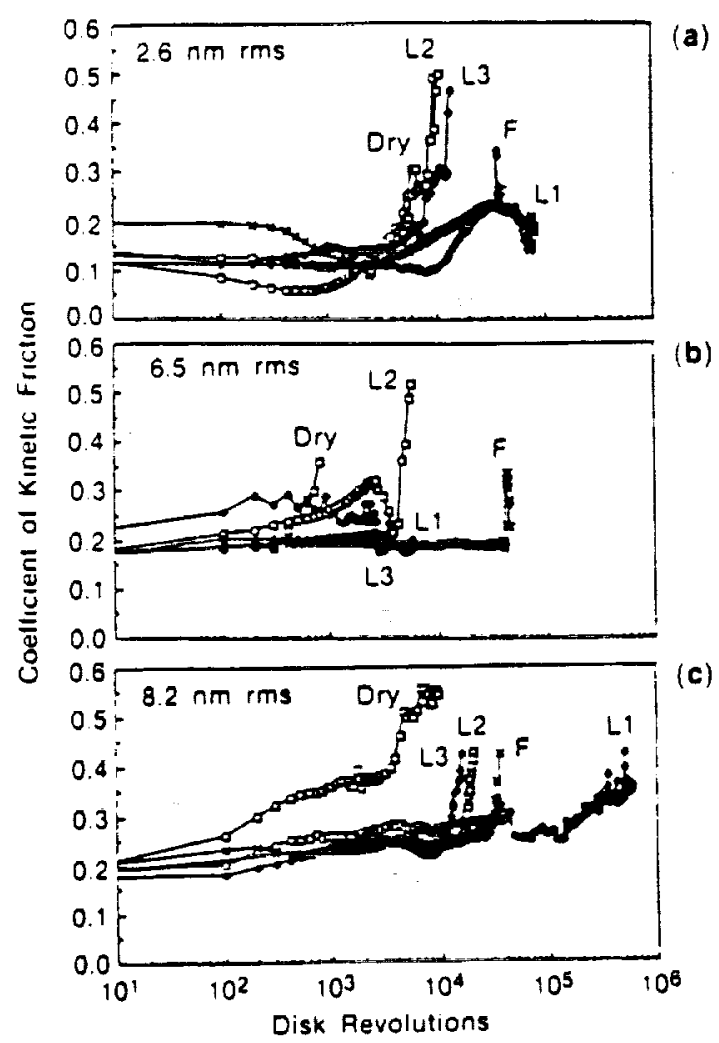

Figure 46. Friction histories during durability tests for unlubricated and lubricated thin-film disks with carhon overcoat sliding against $\mathrm{Al}_{2} \mathrm{O}_{3}$. TiC. sider at $1.2 \mathrm{~m} / \mathrm{s}$ with 3 $\mathrm{nm}$ of lubricant, (a) disk $X_{1}$ roughness $=2.6 \mathrm{~nm} \mathrm{rms}$, (h), disk $X_{2}$ roughness $=4.5 \mathrm{~nm}$ rms. and (c) disk $B_{1}$ roughness $-5.2 \mathrm{~nm}$ rms (Streator ct al., 1991 h). 


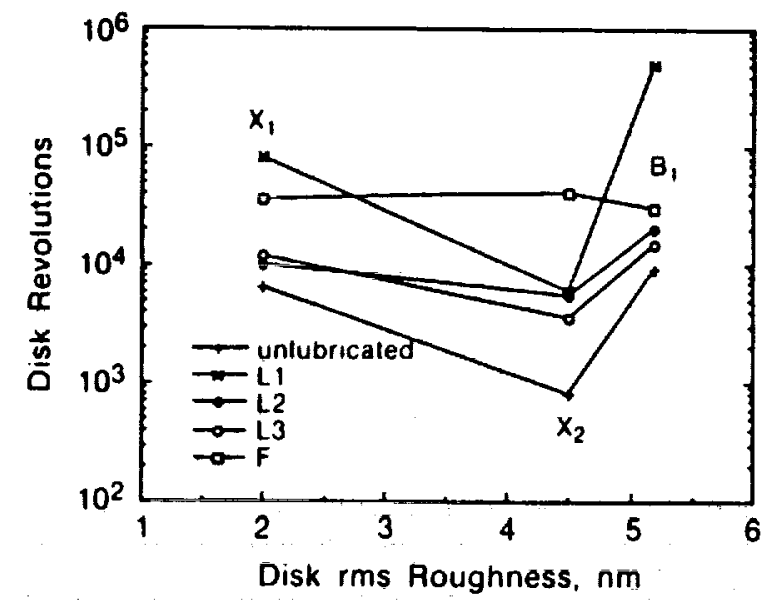

Flgure 47. Disk durability summarized for cach of the sliding convitions of Fig. 4t (Streator et al., 199/b).

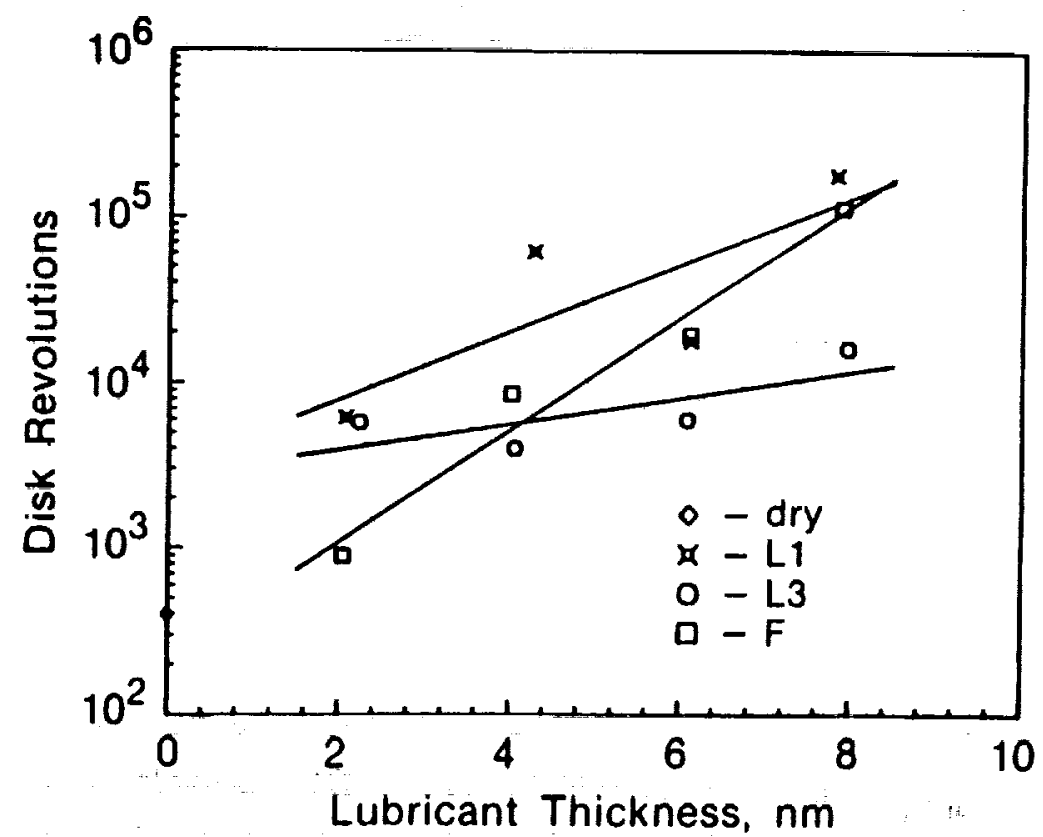

Figur 40. Disk durability as a function of lubricant film thickncss on disk $X_{2}$ (roughness $-4.5 \mathrm{~nm}$ rms) for selected lubricants. Data point at $n \mathrm{~nm}$ is the durability for dry sliding (Streator et al., 199/ b). 


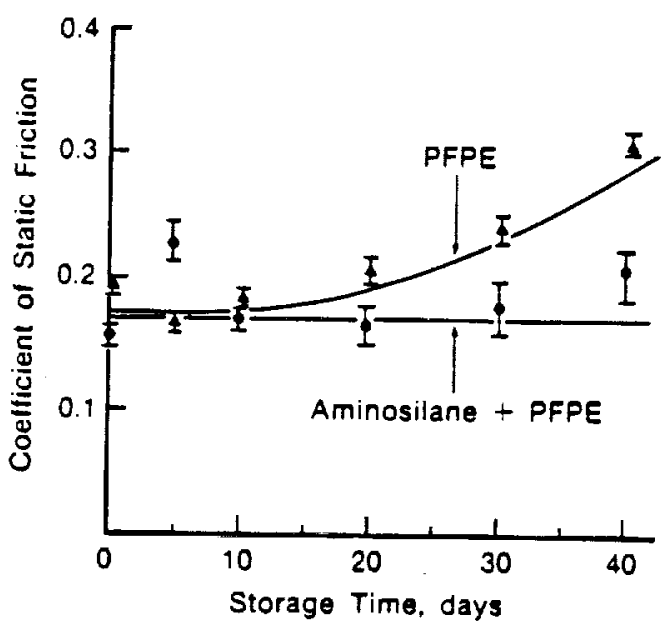

Figan 49. Coefficient of static friction as a function of exposure at $50^{-} \mathrm{C}$ and $10^{-3}$ torr for lubricated spin-coated $\mathrm{SiO}_{2}$ film on a sputtered oxide magnetic film (lloshino et al. 1988).
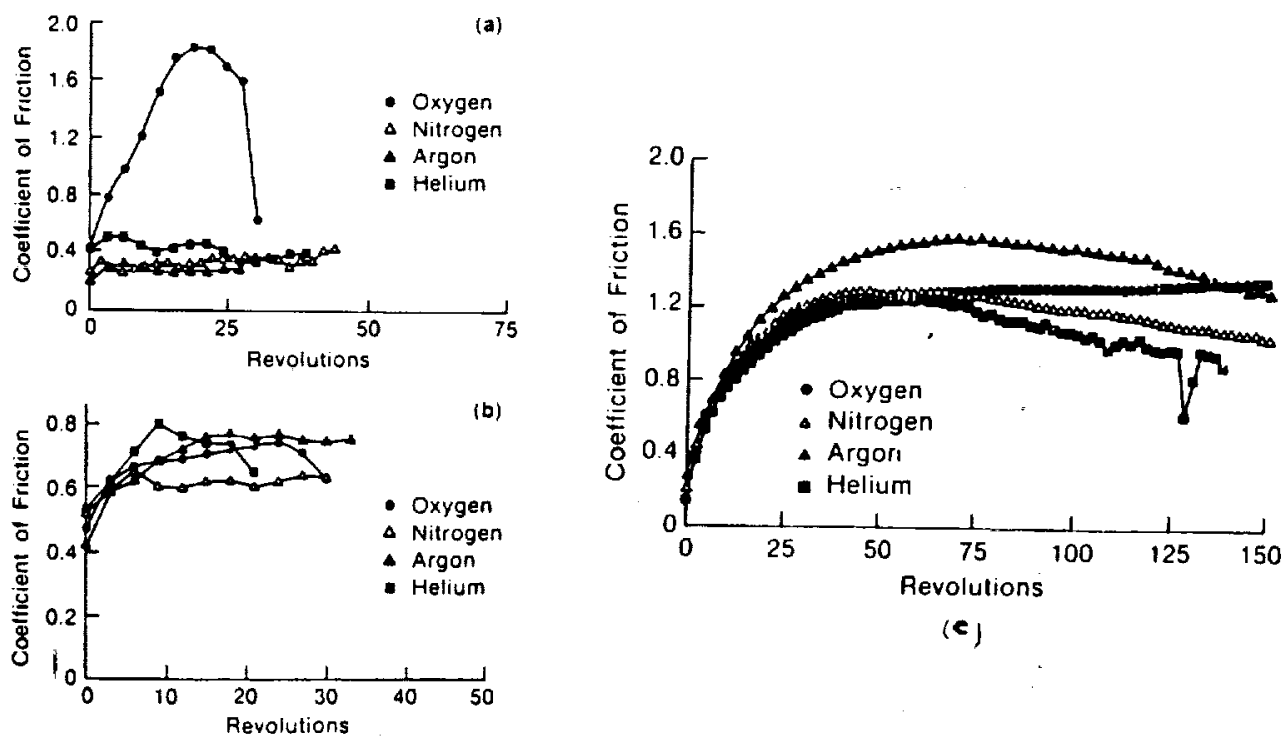

(e)

Figure 50. Coefficient of friction as a function of number of revolutions during sliding of $\mathrm{Al}_{2} \mathrm{O}_{3}$.TiC slider against an unlubricated thin-film disk at a normal load of $150 \mathrm{mN}$ and a sliding speed of $60 \mathrm{~mm} / \mathrm{s}$ (a) disk with carbon overcoat ( $B_{1}$ with no lubricant) in dry gases (b) disk with zirconia overcoat ( $B_{2}$ with no lubricant) in dry gases, and (c) disk with carbon overcoat ( $B_{1}$ with no lubricant) in various gases, all at $4 \%$ RH (Strom et al., 1991). 


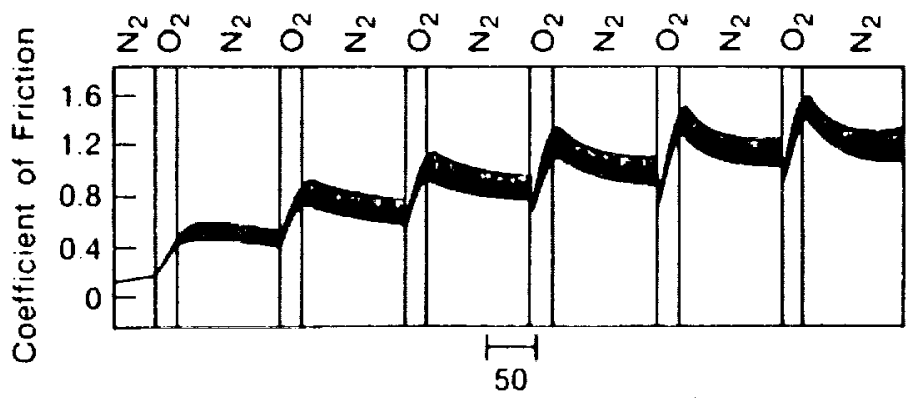

Revolutions

Figure 51. Coefficient of friction as a function of number of revolutions during sliding of $\mathrm{Mn} \cdot \mathrm{Zn}$ ferrite slider against an unlubricated thin-film Jisk at a normal load of 100 $\mathrm{mN}$ and a sliding speed of $60 \mathrm{~mm} / \mathrm{s}$ in various dry gascs, (Marchon ct al., 1990).
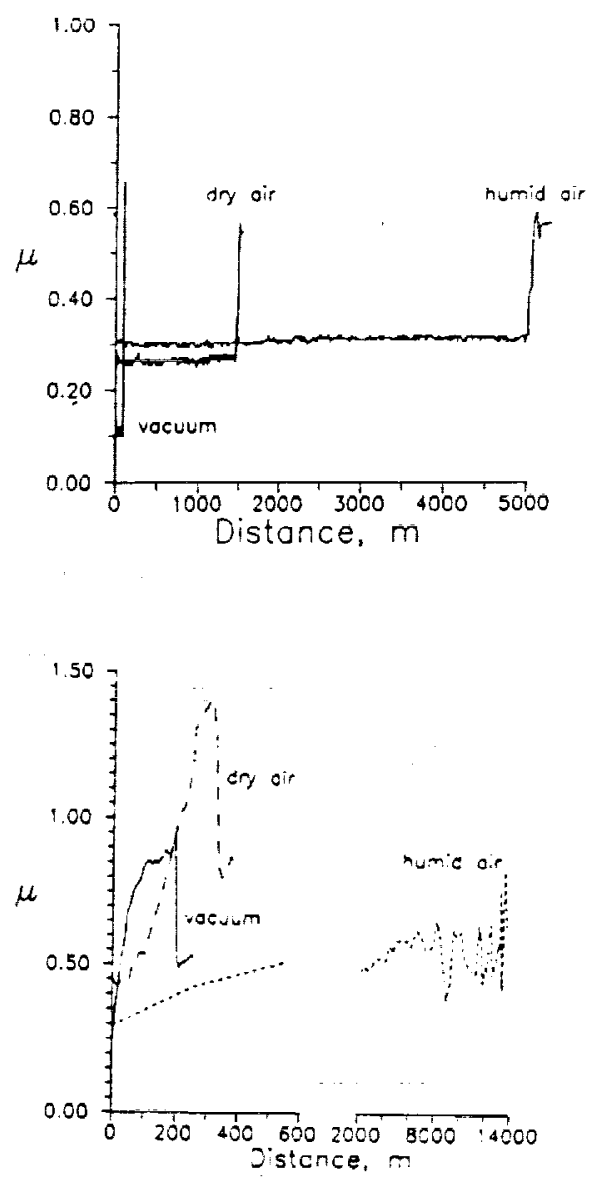

Figure 52. Coefticient of friction ( $\mu$ ) as a function of distance slid for hemispherical pins of Mn-7.n ferrite on lubricated thin-film disks in vacuum. Jry air and air with $50 \%$ RII, at $1 \mathrm{~m} / \mathrm{s}$ sliding speed and $98 \mathrm{miN}$ applied load (a) luhricated thin.film disk with carbon overcoat (disk $B_{1}$ ). (b) lubricated thin-film disk with sirconia overcoat (disk $B_{2}$ ) (Dugger et al., 1992). 

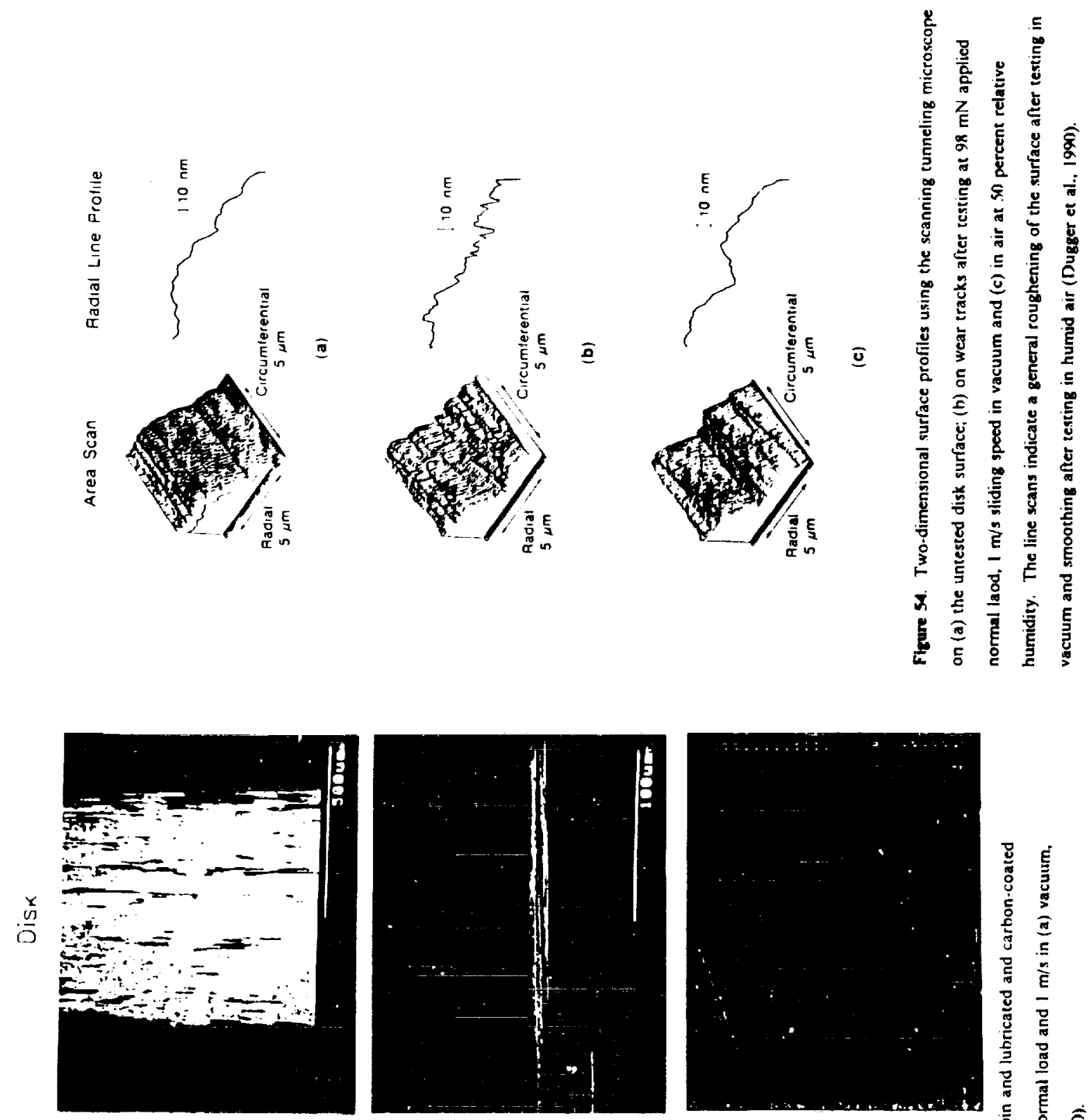
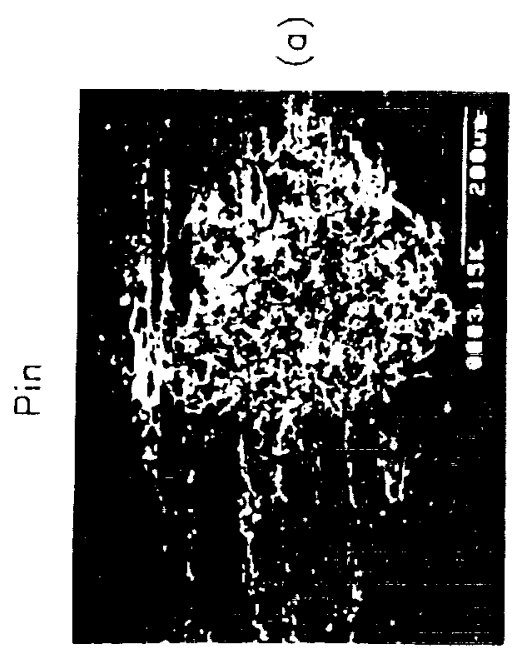

a
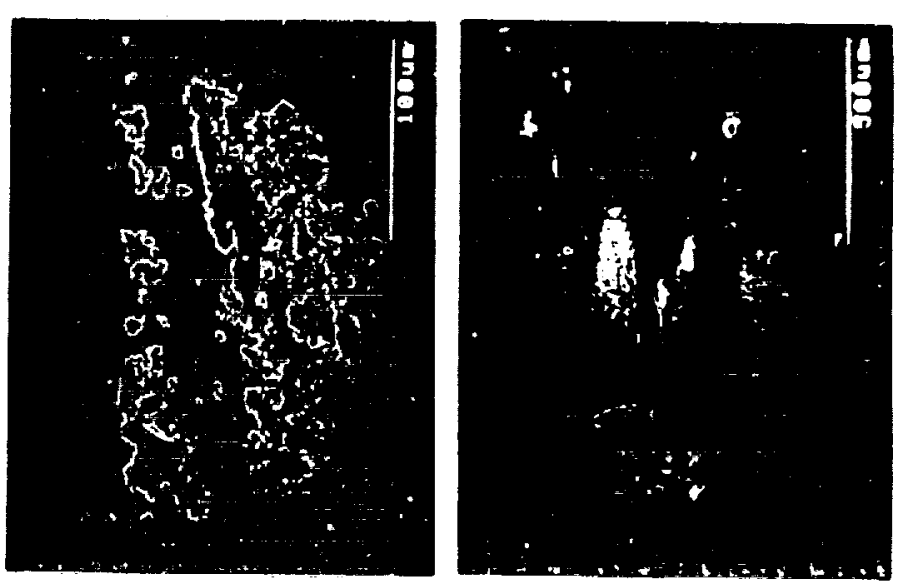

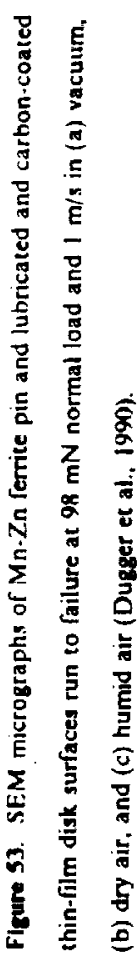




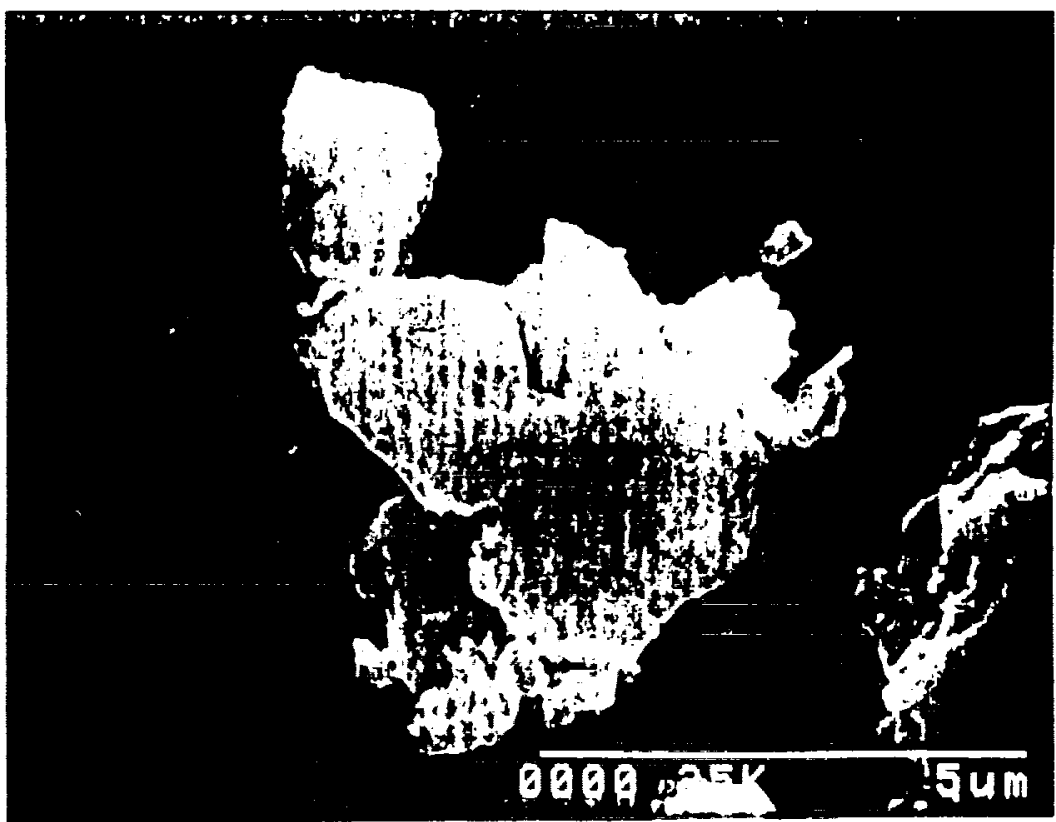

Figure 53. SEM micrograph of an isolated metallic wear particle on the pin from a test at $98 \mathrm{mN}$ applied normal load, $1 \mathrm{~m} / \mathrm{s}$ sliding speed in vacuum, showing evidence of agglomeration (Dugger et al., 1990).

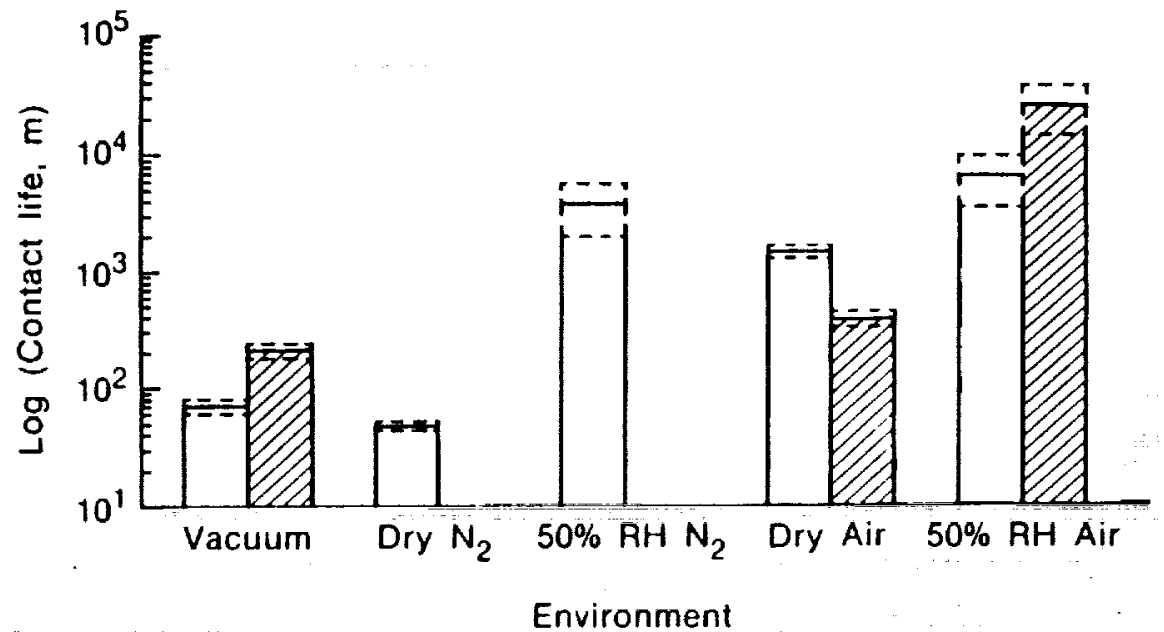

Figure 56. Summary of the contact lives as a function of environment for lubricated thin-film disks with carbon and zirconia overcoats (disks $B_{1}$ and $B_{2}$, respectively) at I $\mathrm{m} / \mathrm{s}$ sliding and $98 \mathrm{mN}$ load in a sliding test with hemispherical pins of $\mathrm{Mn}-7 . \mathrm{n}$ ferrite. Error hars represent the standard deviation from at least four experiments. 

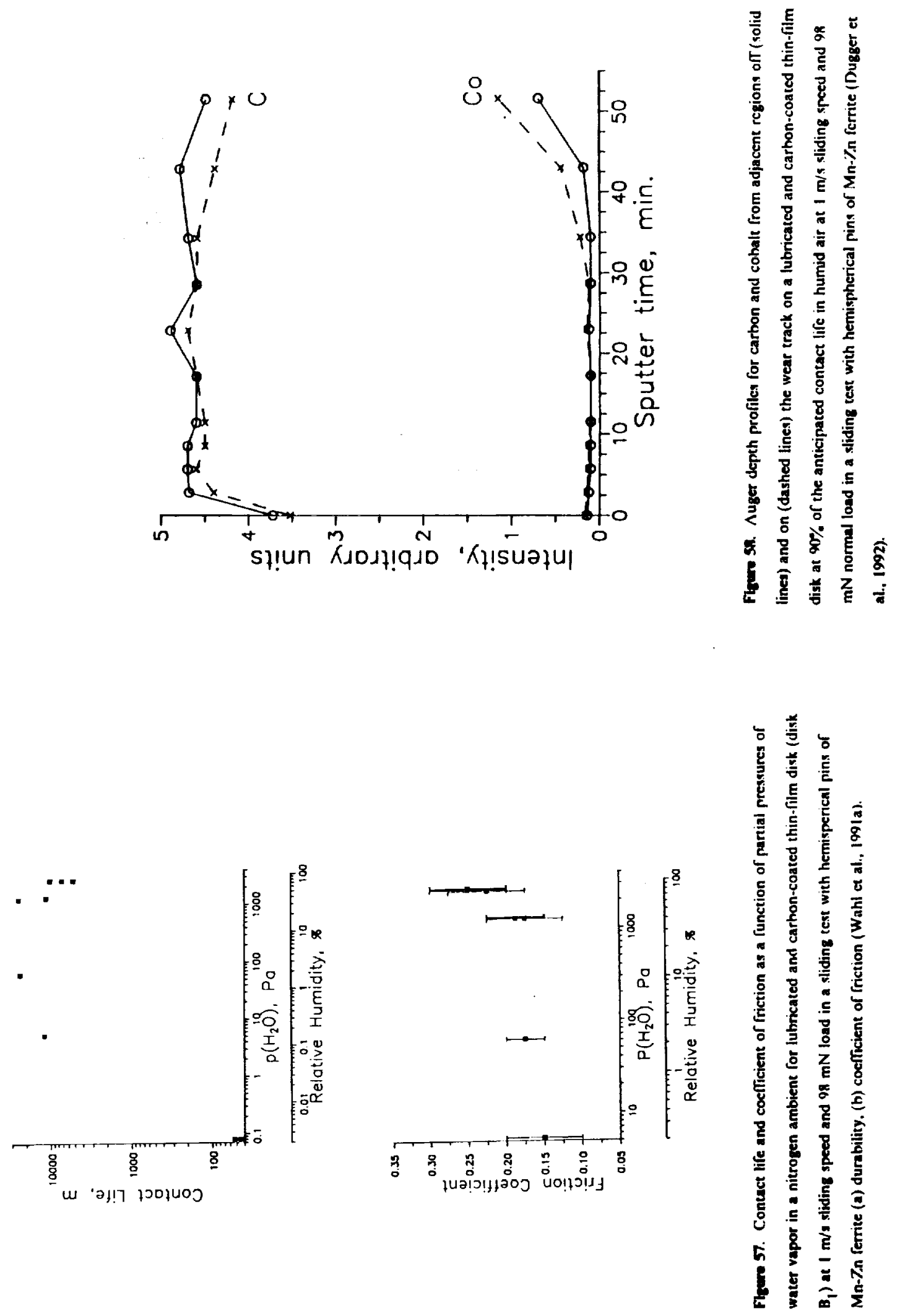


(n)

